\title{
Multiple Photon IR Laser Photophysics and Photochemistry. III
}

\author{
V. N. BAGRATASHVILI, V. S. LETOKHOV, A. A. MAKAROV \\ and E. A. RYABOV \\ Institute of Spectroscopy, USSR Academy of Sciences, 142092, Troitzk, \\ Moscow Region, USSR
}

(Received June 6, 1983)

This paper is the third in series of reviews on multiple photon IR laser photophysics and photochemistry. The main points of multiple photon excitation of molecules at lower discrete levels and in the vibrational quasicontinuum are succesively considered.

\section{Contents}

5. Multiple Photon Absorption and Excitation of Lower Vibrational Molecular Levels ...................... 173

5.1. Multiple photon absorption of IR radiation by molecules ............................. 174

5.1.1. Measuring techniques............... 174

5.1.2. MP absorption for various laser energy fluence and intensity ..................... 178

5.1.3. MP absorption spectra ............... 185

5.2. Spectroscopy of multiple photon excited molecules ... 189

5.2.1. Linear IR absorption (double IR-IR resonance) 190

5.2.2. UV absorption (double IR-UV resonance) .... 193

5.2.3. Raman scattering (double IR-RS resonance) .. 196

5.2.4. IR fluorescence ...................... 199 
5.3. Fraction of molecules involved in multiple photon excitation

5.3.1. Role of collisions in passage of lower vibrational levels

5.3.2. Determination of the $q$ value ........... 209

5.3.3. Experimental results for different molecules ... 212

5.4. Theoretical models of the IR excitation of molecules at lower transitions

5.4.1. Model of one-dimensional anharmonic oscillator 218

5.4.2. Mechanisms of compensation of anharmonic shift at lower transitions ..................

5.4.3. Model of multiphoton transitions to the quasi-

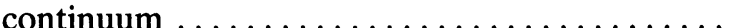

5.4.4. Model of weak transitions

5.4.5. Experimental testing of theoretical models ....

6. Multiple Photon Excitation of Molecules in Vibrational Quasicontinuum..........................

6.1. Experimental study of MP excitation of molecules in the quasicontinuum.

6.1.1. Average level of excitation of molecules in the quasicontinuum.....................

6.1.2. Stochastization of vibration energy in MP excitation of molecules ................... 244

6.1.3. IR absorption spectrum of a highly excited polyatomic molecule ....................

6.2. Comparison of the theory of molecular excitation in the quasicontinuum with experiment.............. 258

6.2.1. Transition cross-sections in the quasicontinuum

6.2.2. Semiempirical model of IR excitation and dissociation........................ 262

6.3. Vibrational energy distribution at IR-MP excitation of

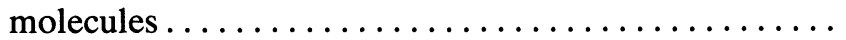

6.3.1. Features of intermolecular distribution nonequilibrium ..................... 268

6.3.2. Causes of the formation of two molecular ensembles ........................

6.3.3. Vibrational distribution in the upper molecular ensemble ......................... 


\section{MULTIPLE PHOTON ABSORPTION AND EXCITATION OF LOWER VIBRATIONAL MOLECULAR LEVELS}

The first stage in MP excitation of molecule is the passage of lower discrete vibrational levels. It is this stage responsible for the resonant character of MP excitation of a polyatomic molecule that provides MP molecular dissociation selectivity-the very important property of the effect.

The difficulty of experimental studies is due to the absence of direct methods which would enable us to follow the excitation dynamics of several lower vibrational molecular levels from fixed initial vibrational-rotational states. The methods of calorimetry of the energy absorbed by molecules from the IR field developed by now allow obtaining MP absorption characteristics just averaged over molecules. Besides, in many cases it is difficult to separate the first stage from the subsequent one in the quasicontinuum using experimental dependence only.

There are material difficulties in the theory developed for quantitative description of MP excitation. In a strong field we should consider multistep and multiphoton transitions from a lot of molecular states at the gas temperature existing in the experiment. Yet, for many polyatomic molecules, especially for heavy molecules with a low rotational constant as well as for asymmetrical top molecules, the vibrational-rotational transition in linear absorption spectra have not been identified so far. Besides, at present there is almost no spectroscopic data for vibrational levels with $v>2 ; 3$ (rotational constants, intramode and intermode anharmonicities, etc.). The shortcoming of spectroscopic information as well as insufficient experimental data on excitation of lower levels-all this makes it very difficult to work out a theory for rather full quantitative description of the process. Most of the developed theoretical models are still a qualitative or semiquantitative.

In this Chapter which continue a series of reviews ${ }^{1,2}$ we deal with the passage of molecules through lower vibrational levels as they are excited by IR laser radiation. A considerable part of information on this problem has been obtained from measuring MP absorption (Section 5.1) and from studying the spectroscopic properties of MP excited molecules (Section 5.2). An important quantitative characteristic is the fraction of molecules $q$ involved into the process of MP excitation. 
The measurements of the $q$ value are discussed in Section 5.3. These data form the basis of correlation with the experimental results and the results of the theoretical models for excitation of lower levels presented in Section 5.4.

\subsection{Multiple photon absorption of IR radiation by molecules}

MP absorption of IR radiation can be observed in various polyatomic molecules in gas phase. But some specific characteristics of the process of MP absorption, such as the dependence of the average energy absorbed by one molecule $\bar{\varepsilon}$ on radiation energy fluence $\Phi$ or radiation frequency $\Omega$ may differ greatly for molecules of different types. For example, the value of radiation energy fluence at which MP absorption of different molecules can be observed ranges very widely from $10^{-3}$ $10^{3} \mathrm{~J} / \mathrm{cm}^{2}$. We are going to give the energy and spectral characteristics of MP absorption for different molecules and particularly for molecules with different numbers of atoms, different symmetry and different structure of vibrational-rotational spectra. First of all, however, we shall consider the experimental methods of research of MP adsorption in molecules now in use.

\subsubsection{Measuring techniques}

The measuring techniques of the value of absorbed energy are based on recording the variations in energy characteristics of exciting IR radiation after its interaction with the molecules (direct calorimetry) as well as the energy state changing of the molecules themselves (opto-acoustic or opto-thermal calorimetry). There is quite a number of more complex spectroscopic methods to study vibrationally excited molecules giving important information on the process of MP excitation. All these methods will be described in Section 5.2.

We must note that relatively a small value of multiphoton absorption cross-section $10^{-19}-10^{-21} \mathrm{~cm}^{2}$ calls for using IR lasers which are able to provide a radiation energy fluence of $1-10^{2} \mathrm{~J} / \mathrm{cm}^{2}$ to realize effective MP excitation of molecules. Besides, in many cases, for example in studying the quantitative characteristics of MP processes, strong radiation focusing cannot be applied. This requires that the laser radiation pulse energy should be $0.1-10 \mathrm{~J}$ which, for now, can be obtained only within some sections of the IR range (see Ref. 1, Section 2.8). 
Direct calorimetry This method is based on measuring the laser pulse transmission through the cell with the gas to be studied (Figure 5.1). By measuring the values of energy fluence $\Phi_{\text {in }}, \Phi_{\text {out }}$ respectively at the input and output of a cell with allowance made for the IR pulse attenuation by the cell windows, we can calculate the value of average absorbed energy per one molecule in the volume under irradiation

$$
\bar{\varepsilon}=\left(\Phi_{\text {in }} \mid N L\right) \ln \left(\Phi_{\text {in }} \mid \Phi_{\text {out }}\right)
$$

where $N$ is the concentration of absorbing molecules in the gas cell and $L$ - length of the cell. This measuring technique of $\bar{\varepsilon}$ is absolute provided that all the energy changing is connected with the radiation absorption in the volume under irradiation, i.e., we can neglect completely the effects like radiation scattering in gas. Since the dependence of absorbed energy $\bar{\varepsilon}$ on $\Phi$ may be essentially nonlinear, in measuring of $\bar{\varepsilon}$ by the method of direct calorimetry we should, as a rule, try to provide radiation energy fluence rather uniform in length and laser beam cross-section. For this purpose, collimated beams of laser radiation are used and the cell length and gas pressure are chosen so that the absorption in the cell is no higher than $10-15 \%$.

One of the shortcomings of this method is, first of all, that it is impossible to measure $\bar{\varepsilon}$ at weak absorption, when a long cell is needed, since it is difficult to ensure a constant value of $\Phi$ because of divergence of laser radiation. Besides, we are not able to carry out measurements when the IR radiation intensity exceeds the threshold of optical damage of the cell windows. The value of threshold radiation intensity for uch IR materials as, for example, $\mathrm{NaCl}, \mathrm{KBr}, \mathrm{Ge}, \mathrm{ZnSe}$, is no higher than $10^{8} \mathrm{~W} / \mathrm{cm}^{2}$ with the pulse duration $\tau_{p}=10^{-7} \mathrm{~s}$ which corresponds to $\Phi \leqslant 10 \mathrm{~J} / \mathrm{cm}^{2}$.

Opto-acoustic calorimetry. The laser IR pulse energy absorbed during MP excitation is converted finally to thermal energy of medium as a result of subsequent relaxation processes. If the rate of vibrational-

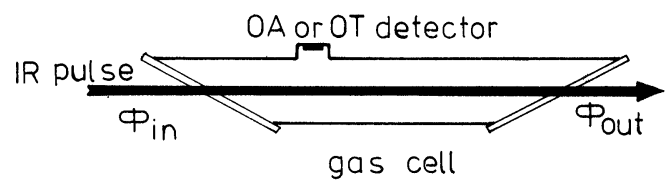

FIGURE 5.1 Calorimetry of absorbed energy at IR-MP excitation of molecules. 
translational relaxation $\left(\tau_{V-T, R}\right)$ exceeds the rate of diffusion of vibrationally excited molecules to the gas cell walls the gas is subjected to thermal heating. This causes the gas pressure to increase rapidly and an acoustic wave to be formed which can be easily recorded by a sensitive condenser microphone (Figure 5.1). This forms the basis for the opto-acoustic (OA) method of recording of MP absorption ${ }^{4}$ (see also monograph ref. 3).

It is easy to choose the conditions under which the electric signal from the opto-acoustic detector is strictly proportional to the IR pulse energy absorbed in the gas. Therefore to measure the value of $\bar{\varepsilon}$ with an opto-acoustic detector we suffice to measure the OA detector sensitivity at a fixed value of $\Phi$, for example, through direct calorimetry. The sensitivity of modern OA detectors with a condenser microphone under pulsed operation enables us to measure absorbed energy $\bar{\varepsilon}$ of no less than $10^{-4}-10^{-3} \mathrm{eV} /$ molec on condition that background signals are suppressed. The dynamic range of $\mathrm{OA}$ detectors by the value may be $6-7$ orders.

The method of OA calorimetry is especially convenient for measuring the value of absorption both at small absorptions and when it is necessary to focus the laser radiation. Laser focusing is essential in measuring MP absorption at radiation intensities exceeding the threshold of damage of the cell windows, in case of low-power lasers and lasers with a high radiation divergence. To perform correct measuring of the $\bar{\varepsilon}$ value in focused laser beams we should discriminate the OA signal responsible only for the absorption in the region of the caustic waist where the $\Phi$ value is constant in length, i.e. provide the spatial resolution of $\mathrm{OA}$ dector. ${ }^{4}$ The possibility of providing the spatial resolution under pulsed operation is possible because $\mathrm{OA}$ signals from different absorption zones along the laser beam reach the microphone and are recorded at different instants of time. Because of this temporal selection of OA signals is possible. According to, ${ }^{5}$ even though we use a multimode TEA- $\mathrm{CO}_{2}$ laser having rather high divergence $\left(10^{-3} \mathrm{rad}\right)$ it is possible to reach spatial resolution of 3-4 $\mathrm{mm}$. Spatial resolution allows effective suppression of the background signal connected with the absorption in the cell windows. This is of particular importance in recording weak absorption at high radiation intensity.

OA measurement of MP absorption has the following essential disadvantage. First, the correct measuring of absorption is possible 
only at low dissociation yields when we are sure of transformation of absorbed energy into a thermal gas energy. Second, the OA detection sensitivity may depend on gas pressure. Its value is essentially determined by the relation of the time of $V-T, R$ relaxation in gas $\tau_{V-T, R}$ and the time of thermal relaxation on the cell walls $\tau_{T}$. The value $\tau_{V-T, R}$ is inversely proportional to gas pressure $p$. The value $\tau_{T}$ depends on the rate of diffusion of vibrationally excited molecules to the cell wall and is proportional to $p$. With $\tau_{V-T, R} \ll \tau_{T}$ the OA detector sensitivity is almost independent on $p$, since the whole absorbed energy is first consumed for gas heating. With $\tau_{V-T, R} \geqslant \tau_{T}$ the sensitivity begins to drop with a decrease of $p$ because of heat scattering on the cell walls. For OA detectors with the cross dimension of their chamber of about $1 \mathrm{~cm}$ the sensitivity drops considerably with $p=5-0.1$ Torr (in dependence on the type of molecule and excitation level).

Opto-thermal calorimetry At rather a low gas pressure the time of diffusion of vibrationally excited molecules to the cell walls is much shorter than the time of $V-T, R$-relaxation. In this case the energy of vibrational excitation of molecules is directly consumed to heat the cell walls. If the wall is made of a material sensitive to temperature variations, the increase of $T$ can provide us with information on the laser radiation energy absorbed in the gas. This forms the basis for the method of optothermal (OT) calorimetry of absorbed energy. ${ }^{6}$ The optothermal detector is more preferential for being used in measuring the absorption right in the region of low pressures of gas $p<0^{-1}$ Torr where the OA detector sensitivitiy drops considerably. An important advantage of the OT detector over the OA detector is that the first can operate under conditions when its sensitivity does not depend on gas pressure. This possibility is explained by the fact, that in case of opto-thermal recording the energy absorbed is always used in heating the walls of the cell containing a sensitive element.

Most of thermal detectors of IR radiation, such as bolometers, pyroelectric detectors, thermistors and thermocouples, can be used as sensitive elements of OT detecors. Pyroelectric detectors are best suited for calorimetry of MP absorption. Their principle of operation is based on variations of spontaneous polarization of its pyroelectric material as its temperature changes, which leads to electric current generation. An important merit of the pyroelectric OT detector is its high recording sensitivity. For example, at a gas pressure of $10^{-2}$ Torr 
it is possible to record absorbed energy on a level no worse than $10^{-3} \mathrm{eV} /$ molec. $^{7}$ Besides, pyroelectric OT detectors have high time resolution (up to $10^{-7} \mathrm{~s}$ ). These merits of pyroelectric OT detectors allow measuring MP absorption in molecular beams as well as performing time-of-flight experiments.

Processing of data on MP absorption measurements In measuring the $\bar{\varepsilon}$ value by the method of direct calorimetry the accuracy depends on the measurement accuracy of absolute values of radiation energy fluence $\Phi$ and the measurement accuracy of transmission of cells filled with gas $\Phi_{\text {out }} / \Phi_{\text {in }}$. At multiple measurement due to averaging we can provide a sufficiently high measurement accuracy of the value $\Phi_{\text {out }} / \Phi_{\text {in }}$. At the same time in measuring the $\Phi$ value there is an ambiguity related mainly to nonuniform distribution of pulse energy $E_{p}$ across the beam. The value $\Phi=E_{p} / S$ depends on the beam cross-section $S$ which is ambiguous. To get rid of this ambiguity in Ref. 8 a method was suggested to determine the true dependences of $\bar{\varepsilon}$ on $\Phi$. This method is based on measuring the distribution shape of pulse energy $E_{p}$ across the beam. If the cross profile of laser beam can be presented as

$$
f(r)=\exp \left[-(r / a)^{n}\right]
$$

then, knowing the parameters $a$ and $n$, and measuring the value $\Phi_{\text {out }} / \Phi_{\text {in }}$ we can find the true value of beam energy fluence $\Phi$ which corresponds to the measured value of transmission.

It is very important to get the true dependences $\bar{\varepsilon}(\Phi)$ not averaged over beam cross-section in measuring absorption in focused laser beams, for example in OA detectors (see Ref. 9). After proper processing of data the main error in determining the dependence of $\bar{\varepsilon}$ on $\Phi$ will depend basically on the error in measuring the absolute value of pulse energy $E_{p}$.

\subsubsection{MP absorption for various laser energy fluence and intensity}

The molecular absorption of IR radiation by its nature may be divided (conventionally to some extent though) into three regions: (1) linear absorption; (2) absorption saturation; (3) MP absorption.

At a low radiation energy density when the interaction of the IR radiation with a molecular system is linear the $\bar{\varepsilon}$ value increases in 
proportion to $\Phi$

$$
\bar{\varepsilon}=\sigma_{0} \Phi
$$

where $\sigma_{0}$ is the linear absorption cross-section.

When the $\Phi$ value becomes comparable with the saturation energy fluence of a vibrational-rotational transition $\Phi_{s}{ }^{10}$ the absorption becomes nonlinear. The saturation kinetics of a molecular vibrational transition under the action of an IR radiation pulse, the frequency of which coincides with the frequency of a vibrational-rotational transition, was considered in ref. 2 (Section 3.3.2).

The characteristic values of the cross-section of molecular allowed vibrational-rotational transitions range from $10^{-14}-10^{-16} \mathrm{~cm}^{2}$. In the case when pulse duration $\tau_{p} \ll \tau_{\text {rot }}$ this corresponds to a range of $\Phi_{s}$ from $10^{-6}-10^{-4} \mathrm{~J} / \mathrm{cm}^{2}$. With $f \tau_{p} \gg \tau_{\text {rot }}$ the characteristic values of $\Phi_{s}$ run between $10^{-4}$ and $10^{-1} \mathrm{~J} / \mathrm{cm}^{2}$.

As noted above, the dependence of absorbed energy on IR radiation pulse energy fluence may differ essentially for different types of molecules. First we are going to consider these dependences for simple molecules.

Figure 5.2 shows the measured dependences of the average number of absorbed quanta $\bar{n}=\bar{\varepsilon} / \hbar \Omega$ and the effective absorption crosssection $\sigma=\bar{\varepsilon} / \Phi$ on IR radiation energy fluence for three-atom molecules $\mathrm{D}_{2} \mathrm{O}$ and OCS. ${ }^{4}$ A heavy-water molecule is excited at a frequency of $1079.65 \mathrm{~cm}^{-1}$ which corresponds to the vibrationalrotational transition $5_{33}-4_{22}$ of the $\nu_{2}$ vibrational band. It may be seen that with $\Phi<10^{-1} \mathrm{~J} / \mathrm{cm}^{2}\left(I<10^{6} \mathrm{~W} / \mathrm{cm}^{2}\right)$ the dependence of $\bar{n}$ on $\Phi$ is almost linear. With $\Phi>10^{-1} \mathrm{~J} / \mathrm{cm}^{2}$ the value of $\bar{n}$ stops increasing reaching $\bar{n}=0.8$ quantum $/$ molecule with $\Phi=1.0 \mathrm{~J} / \mathrm{cm}^{2}$, and the value of $\sigma$ within the range of $\Phi=10^{-1}-10 \mathrm{~J} / \mathrm{cm}^{2}$ decreases by more than an order. As one can see from analyzing the solution of equation (3.72) in ref. 2 the behavior of the dependence $\bar{n}(\phi)$ qualitatively agrees with the behavior of a two-level system. (Good qualitative agreement takes place if $p \tau_{\text {rot }}$ taken to be $10 \mathrm{~ns} \cdot$ Torr). This means that during IR photoexcitation of $\mathrm{D}_{2} \mathrm{O}$ with $\Phi<10 \mathrm{~J} / \mathrm{cm}^{2}$ higher vibrational levels are not excited yet.

Quite a similar dependence of $\bar{n}$ on $\Phi$ can be observed for the OCS molecule, too. The OCS molecule is excited at the frequency $\Omega=$ $1045.02 \mathrm{~cm}^{-1}$ which corresponds to the first overtone of the OCS band $-2 \nu_{2}$. Like in the case of $\mathrm{D}_{2} \mathrm{O}$, in IR photoexcitation of OCS 

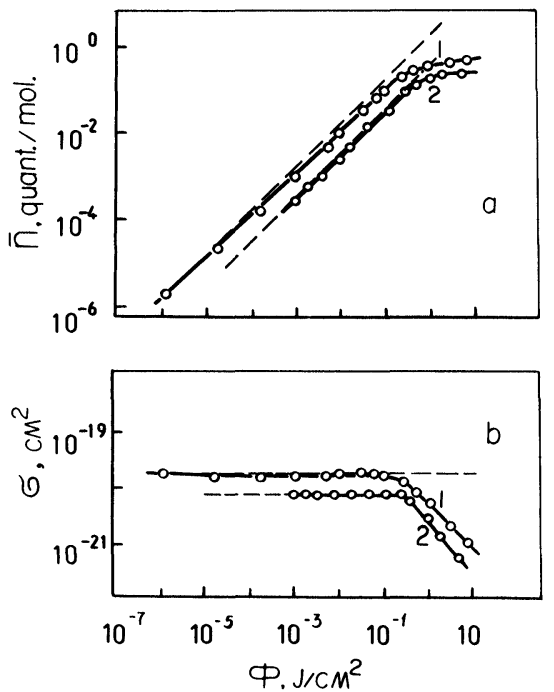

FIGURE 5.2 Dependences of the average number of absorbed quanta $\bar{n}$ and absorption cross-section $\sigma$ on laser energy fluence $\Phi$ for $\mathrm{D}_{2} \mathrm{O}$ (curves 1) and OCS (curves 2) molecules (from Ref. 4).

by pulses with $\phi<10 \mathrm{~J} / \mathrm{cm}^{2}$ the role of multiphoton absorption is probably not essential yet (see, also, ref. 11). It must be noted that, as it follows from the experiment on IR photodissociation of OCS in a molecular beam, ${ }^{12}$ MP absorption in this molecule apparently can take place with $\Phi>50 \mathrm{~J} / \mathrm{cm}^{2}\left(I>1.3 \cdot 10^{8} \mathrm{~W} / \mathrm{cm}^{2}\right)$.

For more complex molecules the dependences $\bar{n}(\Phi)$ (see Figures 5.3-5.6) materially differ from those predicted by a two-level model. This is explained by the process of excitation of higher vibrational levels, that is MP excitation. For the $\mathrm{SF}_{6}$ molecule, for example, difference from the approximation of a two-level system can be observed already when the energy fluence $\Phi>10^{-5} \cdots 10^{-4} \mathrm{~J} / \mathrm{cm}^{2}$ (Figure 5.3). In this case the dependence of $\bar{n}$ on $\Phi$ has no section of full saturation and one can observe continuous change-over from linear absorption to MP absorption.

Even at low energy fluences of IR radiation a certain part of molecules is excited to higher $(\nu>2)$ vibrational states. This conclusion was first drawn in work ${ }^{13}$ on two-frequency dissociation of the $\mathrm{SF}_{6}$ molecule. In Ref. 13 it has been shown that with $\Phi>6 \cdot 10^{-3} \mathrm{~J} / \mathrm{cm}^{2}$ 
MULTIPLE PHOTON IR LASER PHYSICS. CHEMISTRY. III
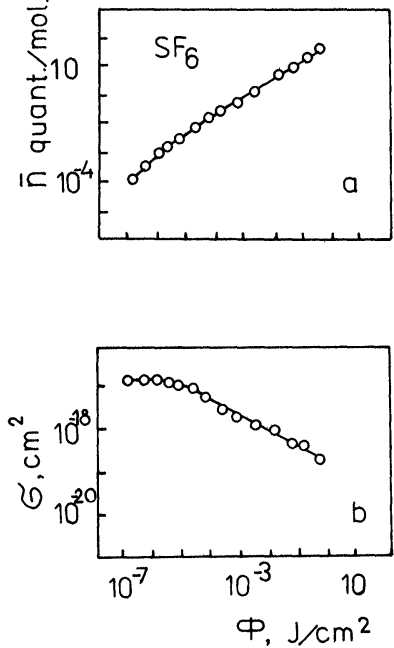

FIGURE 5.3 Dependences of the average number of absorbed quanta $\bar{n}$ and absorption cross-section $\sigma$ on laser energy fluence $\Phi$ for $\mathrm{SF}_{6}$ molecule (from Ref. 4).

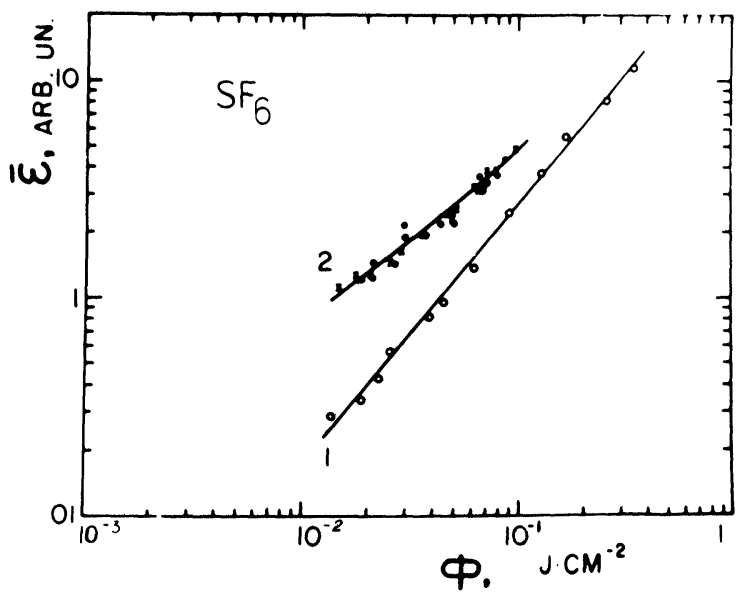

FIGURE 5.4 Dependence of the average absorbed energy $\bar{\varepsilon}$ on laser energy fluence $\Phi$ for $\mathrm{SF}_{6}$ molecule at various IR radiation pulse length $\tau_{p}$ (see text) (from Ref. 16). 


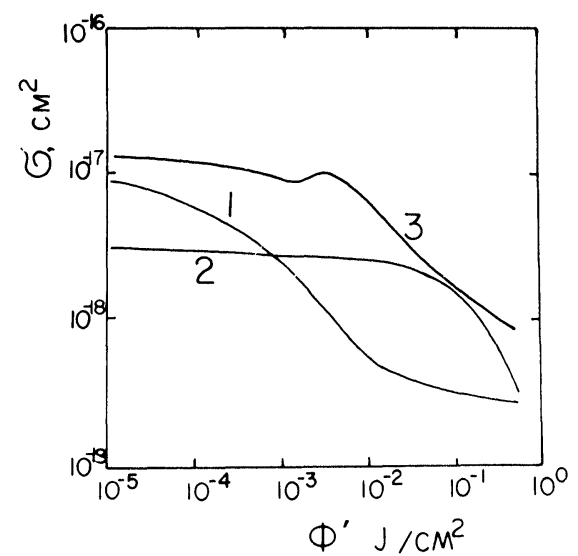

FIGURE 5.5 Dependences of absorption cross-sections $\sigma$ on laser energy fluence: (1) $\mathrm{SF}_{6}$ (from Ref. 18); (2) $\mathrm{SF}_{5} \mathrm{NF}_{2}$ (from Ref. 19); (3) $\mathrm{S}_{2} \mathrm{~F}_{10}$ (from Ref. 8).

$\left(I>6 \cdot 10^{4} \mathrm{~W} / \mathrm{cm}^{2}\right)$ a fraction of molecules is excited to the quasicontinuum and hence absorbs several (3-5) quanta. Since the value of power broadening with $I=6 \cdot 10^{4} \mathrm{~W} / \mathrm{cm}^{2}$ for $\mathrm{SF}_{6}$ molecule $\gamma \approx 0.03 \mathrm{~cm}^{-1}$ is much smaller than the value of anharmonic shift $2\left(X_{33}\right)=3.7 \mathrm{~cm}^{-1}$ it is impossible to explain MP absorption for $\mathrm{SF}_{6}$ under these conditions only by power broadening. Therefore, it has been proposed that there is "soft compensation of anharmonicity", i.e. a mechanism that provides purely radiative excitation of molecules to the quasicontinuum even at low IR radiation intensities. Possible mechanisms of "soft compensation of anharmonicity" are under detailed discussion Section 5.4.

We should note that MP absorption in polyatomic molecules at low levels of excitation is usually observed within the entire absorption band, though the rate of absorbing of vibrational energy by a molecule at different radiation frequencies may differ greatly. For example, the value of $\bar{\varepsilon}$ during excitation in the $P$-branch of linear absorption band with increasing $\Phi$ usually grows faster than in the $R$-branch. ${ }^{4}$ This provides deformation of the absorbed energy spectrum to the longwave side which is considered below in Section 5.1.3.

A continuous increase in the $\bar{\varepsilon}$ value at room temperature, as it has been pointed out, is the common feature of the dependence of $\bar{\varepsilon}$ on $\Phi$ almost for all polyatomic molecules. The dependence of $\bar{\varepsilon}$ on $\Phi$ 
MULTIPLE PHOTON IR LASER PHYSICS. CHEMISTRY. III
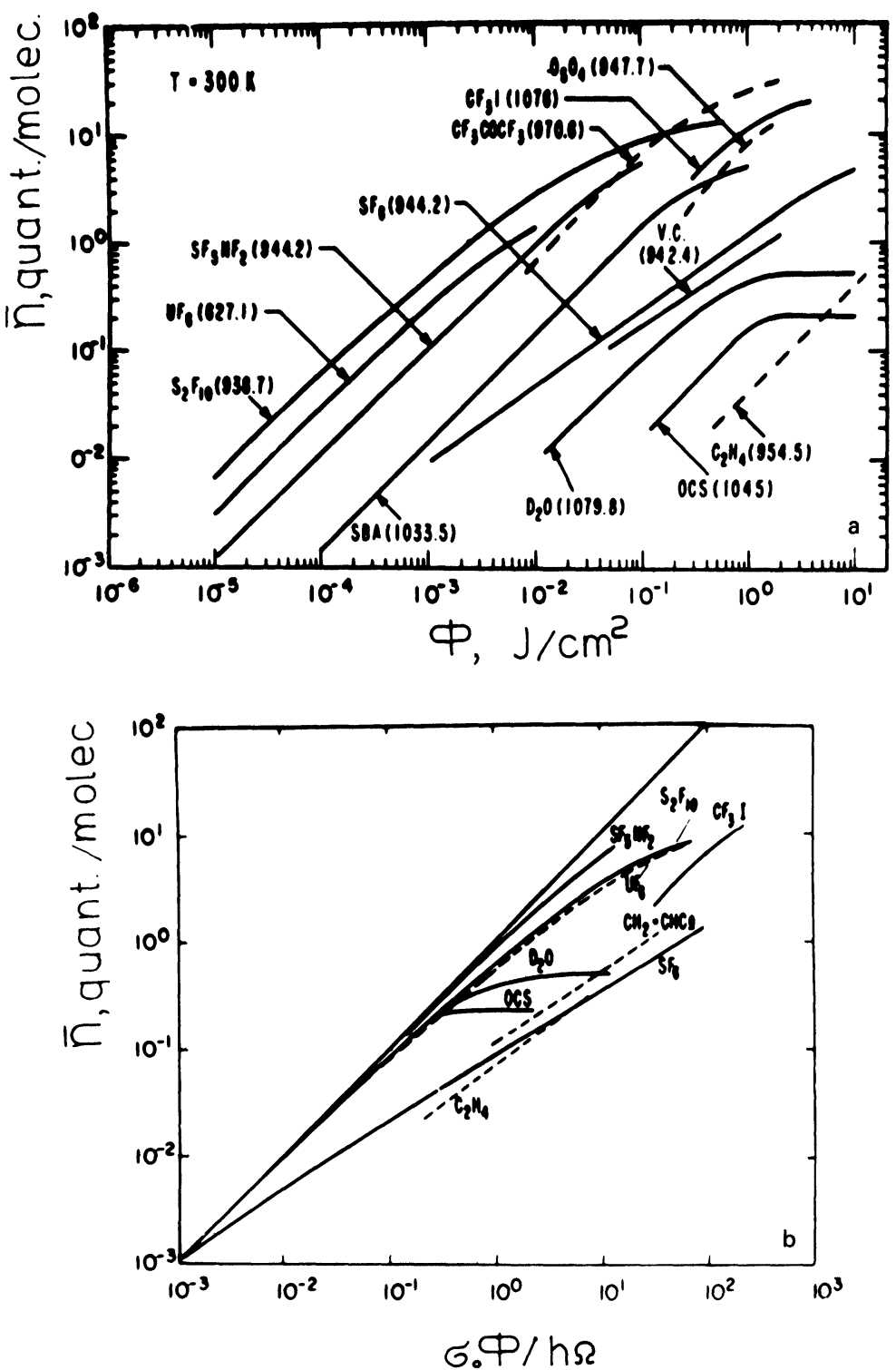

FIGURE 5.6 (a) Comparison of $\bar{n}(\Phi)$ characteristics for various polyatomics at room temperature; (b) Normalized dependences of $\bar{n}$ on $\sigma_{0} \Phi / h \Omega$ for various polyatomics (from Ref. 20). 
for not large molecules is usually slower than the linear one. Yet, there are some exceptions here. First, in the $\mathrm{OsO}_{4}$ molecule the dependence of $\bar{n}$ on $\Phi$ is stepwise and has several sections of saturation. ${ }^{14}$ The authors of work ${ }^{14}$ relate it to the stepwise dependence on $\Phi$ the fraction of molecules interacting with the IR field. Besides, in the $\mathrm{SF}_{6}$ molecule at reduced temperatures the dependence of $\bar{\varepsilon}$ on $\Phi$ at some frequences of radiation is faster than the linear one. In Ref. 15 this is explained by the dominant role of direct two-photon excitation processes of the lower vibrational levels of the molecule. It should be noted that the value of absorbed energy can depend not only on energy fluence but also on exciting IR pulse intensity. ${ }^{16}$ Figure 5.4 shows the dependences of $\bar{\varepsilon}$ in $\mathrm{SF}_{6}$ on $\Phi$ in case of excitation by pulses of two different durations: $\tau_{\mathrm{p}}=600 \mathrm{~ns}$ (long pulse) and $\tau_{p}=50 \mathrm{~ns}$ (short pulse). These dependences are measured by the method of opto-thermal calorimetry. ${ }^{7}$ Rather low gas pressures $(13 \mathrm{~m}$ Torr) in the both cases provide collisionless excitation. It may be seen that in the absence of rotational relaxation at a fixed value of $\Phi$ an increase in intensity causes $\bar{\varepsilon}$ to increase. The influence of intensity conditioned by power broadening is most essential at low energy fluences $(\Phi \leqslant$ $\left.10^{-2}-10^{-1} \mathrm{~J} / \mathrm{cm}^{2}\right)$. With $\Phi>10^{-1} \mathrm{~J} / \mathrm{cm}^{2}$ the difference between the energies absorbed from the short and long pulses is not higher than $30 \%$.

Some features of the process of MP absorption can be observed by comparing the dependences of $\sigma$ on $\Phi$ for the molecules from the homologous series of sulfur fluorides $\mathrm{SF}_{6}, \mathrm{SF}_{5} \mathrm{NF}_{2}, \mathrm{~S}_{2} \mathrm{~F}_{10}$ (Figure 5.5). ${ }^{17-19,18}$ The radiation frequencies $\Omega$ in all the cases are nearby the maxima of the corresponding absorption bands. It is evident that for the $\mathrm{SF}_{6}$ molecule the value of absorption cross-section decreases with an increase in radiation energy fluence approximately as $\Phi^{-1 / 3}$ over the range of variation of $\Phi$ from $10^{-6}-10^{-1} \mathrm{~J} / \mathrm{cm}^{2}$. At the same time for the $\mathrm{SF}_{5} \mathrm{NF}_{2}$ molecule the $\sigma$ value over the range from $10^{-6}-10^{-1}$ is almost constant and begins to drop with $\Phi>10^{-1} \mathrm{~J} / \mathrm{cm}^{2}$ when the dissociation of the molecules becomes essential. In case of the $\mathrm{S}_{2} \mathrm{~F}_{10}$ molecule in the absence of effective dissociation $(\Phi<$ $10^{-3} \mathrm{~J} / \mathrm{cm}^{2}$ ) the value of $\sigma$ almost does not depend on radiation energy fluence which (see Ref. 8) is apparently conditioned by a high density of vibrational levels in the region $1000-2000 \mathrm{~cm}^{-1}$. Because of this the cross-section of purely resonant transitions is not reduced up to the dissociation limit. 
It should be noted that week dependence of absorption cross-section on energy fluence is a quite characteristic feature of MP excitation of large molecules with low quasicontinuum limit. At the same time for molecules with a higher limit of vibrational quasicontinuum $\mathrm{SF}_{6}$, $\mathrm{CF}_{3} \mathrm{I}, \mathrm{BCl}_{3}, \mathrm{OsO}_{4}, \mathrm{C}_{2} \mathrm{H}_{4}$, etc.) the one-frequency field due to vibration anharmonicity cannot provide an exact resonance in the sequence of transitions between discrete levels and this, of course, brings about a decrease in absorption cross-section as the radiation energy fluence increases.

As it may be seen from studying of MP absorption of a great number of different polyatomic molecules there are rather common regularities in the behavior of absorbed energy varying with radiation energy fluence, for example a continuous transition from linear absorption to MP absorption, the absence of sharp dependences of $\bar{\varepsilon}$ on $\Phi$ over a wide range of $\Phi$. At the same time the efficiency of MP absorption for different molecules differs very greatly (Figure $5.6 \mathrm{a}^{20}$ ).

It is evident that one of the values determining the efficiency of MP absorption must be linear absorption cross-section $\sigma_{0}$. This follows, for example, from a considerable difference between the values of MP absorption ${ }^{21}$ and dissociation yields, ${ }^{22}$ in the bands of fundamental frequencies and overtones. Figure 5.6b shows the dependence of the $\bar{\varepsilon}$ value for different polyatomic molecules on $\Phi$, which is normalized to their linear absorption cross-section. It may be seen that the discrepancy in the dependences of $\bar{\varepsilon}$ (or $\sigma \Phi$ ) on $\sigma^{0} \Phi$ for different molecules is much smaller than in the dependences of $\bar{\varepsilon}$ on $\Phi$. To understand this problem properly we must also consider the MP excitation of molecules in the region of quasicontinuum (see Chapter 6).

\subsubsection{MP absorption spectra}

The dependence of the average energy absorbed by a molecule $\bar{\varepsilon}$ on the frequency of exciting IR radiation $\Omega$ is another important characteristic of the process of MP-IR excitation of molecules. Below the dependence $\bar{\varepsilon}(\Omega)$ is called, the multiple photon absorption spectrum.

At very low excitation levels, when the interaction of the molecules with the IR field is linear, the MP absorption spectrum coincides with the linear absorption spectrum of the molecule and is independent on IR radiation intensity. The vibrational-rotational spectrum of linear 
absorption is determined by the transitions just between the lower vibrational levels of the molecule. On the contrary, MP absorption depends on the sequence of many vibrational-rotational transitions in a strong IR field from the lowest to the highest vibrational levels. That is why the MP absorption spectrum, differs from the linear absorption spectrum greatly.

The most important property of MP absorption spectra is that even at large values of $\Phi$ when the molecular excitation level is high enough the MP absorption spectrum, as a rule, is localized near its corresponding band of linear absorption. Thus, the MP excitation of high molecular vibrational levels is resonant by nature. Like in linear spectroscopy, it is high-resolution MP absorption spectra measured by lasers with continuous frequency tuning and a narrow spectral radiation line that must be most informative. Such spectra have been so far produced just for several molecules (see, for instance, Refs. 23 and 24) with the use of a high-pressure tunable $\mathrm{CO}_{2}$ laser. Most of the measurements of the spectral characteristics of MP excitation have been taken using TEA-CO $\mathrm{CO}_{2}$ lasers with discrete frequency tuning with an interval of $1-2 \mathrm{~cm}^{-1}$. These measurements produce, in essence, just the general form of spectrum which, nevertheless, helps us to understand many regularities of the process of MP excitation.

Since the MP absorption cross-section depends on radiation energy fluence it is necessary to maintain with a sufficient accuracy the value of $\Phi$ at a fixed level in measuring MP absorption spectra with the laser radiation frequency scanned. In the general case the value of absorbed energy $\bar{\varepsilon}$ may depend also on the width of the spectrum and the radiation pulse shape. Therefore, in frequency scanning one should also see that these parameters are constant. In the $\mathrm{CO}_{2}$ laser, for example, a strong dependence of the gain on frequency may cause substantial changes in the temporal and spectral parameters of radiation pulse in frequency scanning and, as a result the MP absorption spectrum to be distorted.

Let us take the $\mathrm{SF}_{6}$ molecule to consider the main regularities in MP absorption spectra.

Effect of laser energy fluence The triple-degenerated $\nu_{3}$ mode is the strongest mode in IR absorption for the $\mathrm{SF}_{6}$ molecule. The linear absorption spectrum of the vibrational-rotational band $\nu_{3}$ (see Ref. 1, Figure 2.4) lies in the region of generation of the $P$-branch in the band $00^{\circ} 1-10^{\circ} 0$ of $\mathrm{CO}_{2}$ laser. 
MP absorption spectra of the $\mathrm{SF}_{6}$ molecule measured by the optoacoustic method at room temperature and at different energy fluences have already been shown (Section 4.2.2). With an increase in IR radiation energy fluence one can observe a monotonous shift of the absorption maximum towards the long-wave side and an absorption band broadening. Similar results were obtained in measuring the MP absorption spectrum by the method of direct calorimetry. ${ }^{25}$ Qualitatively the shift and the broadening of the absorption band with increasing intensity is explained by the presence of intramodal and intermodal vibration anharmonicity. Indeed, with an increase in IR field intensity the average level of molecular excitation and hence the contribution of higher vibrational transitions to absorption increase. Since the spectrum of the transition between high levels is shifted towards the long-wave side due to anharmonicity, as the number of transition grows, this brings about a monotonous shift and broadening of MP absorption band. It should be noted that a more substantial "red" shift $\left(70-80 \mathrm{~cm}^{-1}\right)$ can be observed in two-frequency excitation of the molecule (Figure 5.7).

Effect of gas temperature Molecular gas temperature $T$ dictates the initial equilibrium distribution of molecules over rotational and vibrational degrees of freedom, and variations in $T$ result in essential changes in the linear absorption spectra. In case of the $\mathrm{SF}_{6}$ molecule at room temperature, for example, only $30 \%$ molecules are in the

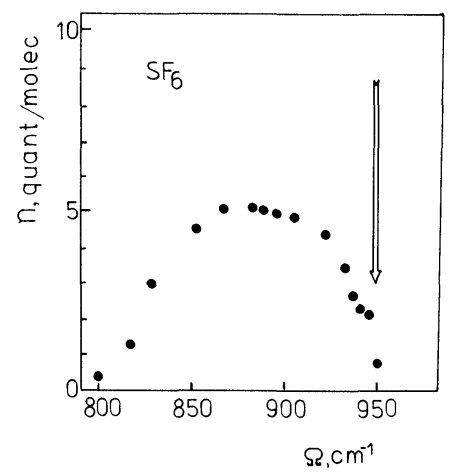

FIGURE 5.7 Dependence of the average number of absorbed quanta from nonresonant field $\Omega_{2}$ on its frequency for $\mathrm{SF}_{6}$ molecules, excited by the resonance pulse $\left(\Omega_{1}\right) ; \Phi_{1}=3 \mathrm{~J} / \mathrm{cm}^{2}, \Omega_{1}=944.2 \mathrm{~cm}^{-1}, \Phi_{2}=0.1 \mathrm{~J} / \mathrm{cm}^{2}$; arrow indicates the maximum of linear absorption spectrum for $\nu_{3}$ mode of $\mathrm{SF}_{6}$ molecule (from Ref. 26). 
ground vibrational state and the rest of them are distributed over low-lying vibrational states in accordance with the Boltzmann distribution. When the $\mathrm{SF}_{6}$ molecules are cooled this causes the spectrum to be narrowed due to a decrease in intensity of "hot" bands as well as rotational distribution width. Besides, during cooling the structure of high-resolution $\mathrm{SF}_{6}$ spectra becomes more pronounced (see Ref. 1, Figure 2.6).

We can naturally expect that temperature variations must affect the spectra of MP absorption, too. Figure 5.8 shows the temperature evolution of the MP absorption spectra of $\mathrm{SF}_{6}$ at two different values of radiation energy fluence. ${ }^{27}$ It may be seen that with an increase in $T$ the absorption from the long-wave side increases considerably and the spectrum is "red"-shifted. The absorption from the short-wave side of the spectrum in this case varies slightly (see also ref. 28). It should be noted that an increase in temperature affects especially

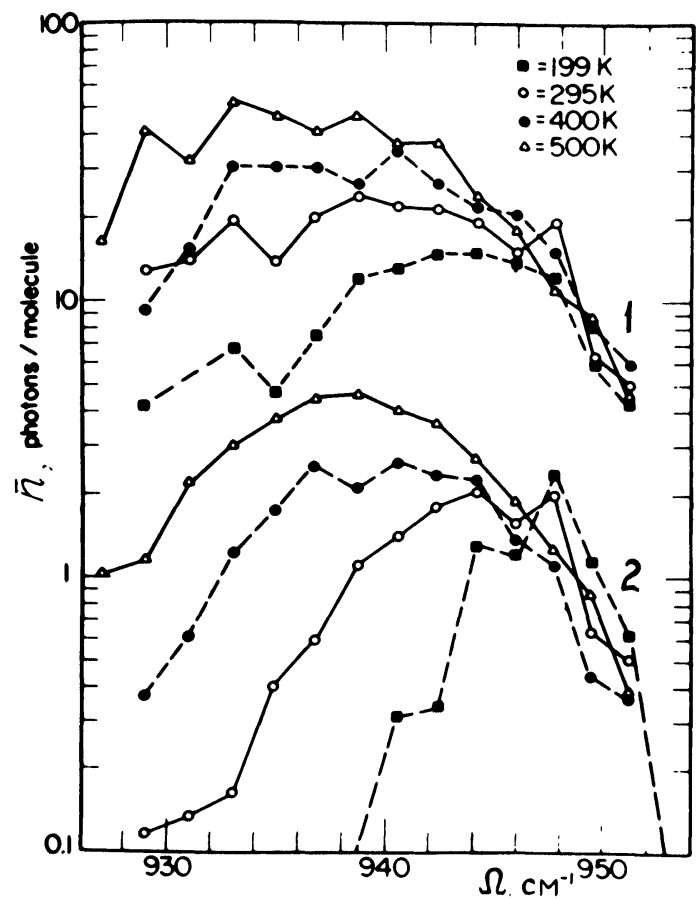

FIGURE 5.8 Spectra of MP absorption for $\mathrm{SF}_{6}$ molecule at various temperatures: $p_{\mathrm{SF}_{6}}=0.5$ Torr; curves (1) $\Phi=0.6 \mathrm{~J} / \mathrm{cm}^{2}$; curves (2) $\Phi=0.02 \mathrm{~J} / \mathrm{cm}^{2}$ (from Ref. 27). 
greatly the MP absorption spectrum at low radiation energy fluences.

The temperature evolution of MP absorption spectra observed (like in case of linear absorption spectra) is caused mainly by the "hot" bands which due to intermodal anharmonicity are displaced towards the long-wave side about the spectrum of the fundamental band $\nu_{3}$ $v=0 \rightarrow v=1$. It is interesting to note here that the integral intensity of MP absorption spectra

$$
S=\int_{\text {Band }} \sigma(\Omega) d \Omega
$$

grows with an increase in $T$ almost exponentially in a range of $\Phi$ from $5 \cdot 10^{-3}$ to $0.6 \mathrm{~J} / \mathrm{cm}^{2}$. At the same time it is $\mathrm{known}^{29}$ that in linear absorption spectra the integral intensity is independent on temperature.

One more effect of temperature and "hot" bands is smoothing of the fine structure of MP absorption spectra. This structure shows up well in the spectra of high-resolution MP absorption $\left(10^{-1}\right.$ to $10^{-2} \mathrm{~cm}^{-1}$ ) of cooled $\mathrm{SF}_{6}{ }^{24}$ According to Ref. 24, a sharp-resonance structure appears due to two-photon absorption at anharmonic splitting of the $\nu_{3}$ mode of $\mathrm{SF}_{6}$. This can be confirmed by the dependence of the energy absorbed in $\mathrm{SF}_{6}$ on $\Phi$ which is faster than the linear one and can be observed at some frequencies. ${ }^{15}$ At room and higher temperatures a weak structure in the MP absorption spectrum of $\mathrm{SF}_{6}$ manifests itself only at low energy fluences $\Phi<0.1 \mathrm{~J} / \mathrm{cm}^{2}$; at higher $\Phi$ it almost disappears.

Here we considered some characteristic features of MP absorption spectra for the $\mathrm{SF}_{6}$ molecules. At present such spectra have been studied for several molecules, such as for example $\mathrm{S}_{2} \mathrm{~F}_{10},{ }^{8} \mathrm{OsO}_{4},{ }^{14}$ $\mathrm{C}_{2} \mathrm{H}_{4}{ }^{4,23}$ The specific shape of MP absorption spectrum strongly depends, of course, on the kind of molecule and the conditions of its excitation. But such features of the spectra as "red" shifts and smoothing of bands with an increase in radiation energy fluence and temperature are quite characteristic of many molecules and result from intramodal and intermodal anharmonicity of molecular vibrations.

\subsection{Spectroscopy of multiple photon excited molecules}

The increase of molecular internal energy resulting from MP absorption may bring about essential changes in the spectroscopic properties 
of molecules. Therefore, important information on different stages of MP excitation process can be obtained using several methods of spectroscopic diagnostics (see Figure 5.9). Below we shall discuss these methods and the results obtained which deal mainly with the passage of lower vibrational levels.

\subsubsection{Linear $I R$ absorption (double $I R-I R$ resonance)}

The method of double IR-IR resonance consists of recording the changes in the IR absorption spectrum as the molecules are excited by IR radiation.

Changes in the IR vibrational-rotational absorption spectra during vibrational excitation of the molecule can take place for quite a number of reasons: the presence of vibration anharmonicity, interaction of vibrations and rotations, interaction of different vibrational modes, a difference in rotational constants for different vibrational levels, variations in symmetry, etc. Therefore the method of double IR-IR resonance (together with the method of double IR-microwave resonance) is widely used in studying different spectroscopic parameters of lower excited vibrational molecular levels. ${ }^{30}$

In one-photon vibrational excitation of a molecule, when the population of the vibrational levels with $v>1$ may be neglected, the vibra-

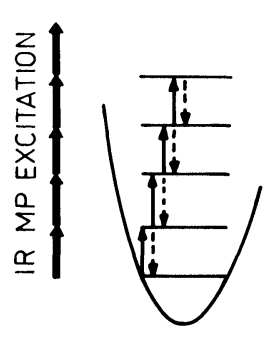

IR

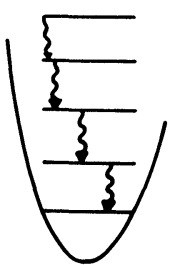

IR

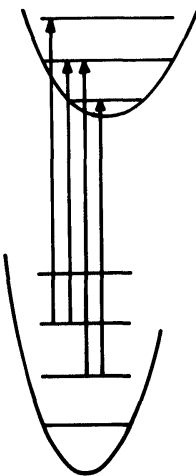

UV ABSORPTION

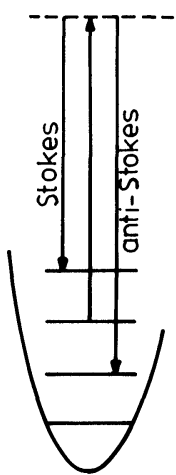

SPONTANEOUS RS

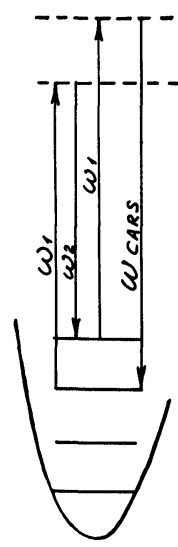

CARS ABSORPTION

FLUORESCENCE

FIGURE 5.9 Methods of spectroscopic diagnostics of MP excited molecules. 
tional-rotational spectrum of double IR-IR resonance is totally determined by the transitions $v=0 \rightleftarrows v=1$ and $v=1 \rightarrow v=2$. In MP excitation when higher vibrational levels of the molecule can be populated effectively the double resonance spectrum is determined by the whole complex of the transitions $v=0 \rightleftarrows v=1 \cdots v=$ $i-1 \rightleftarrows v=i$. Moreover, as the excitation level increases the number of transitions forming the spectrum of double IR-IR resonance is increased. When the molecule is excited to the quasicontinuum the double resonance spectrum must be materially affected by anharmonic interaction of different vibrational modes of the molecule. As there is almost no spectroscopic information on the high vibrational levels of polyatomic molecules it is rather difficult to carry out full quantitative interpretation of double IR-IR resonance spectra (as well as the absorbed energy spectra considered in Section 5.1.3) even at low excitation levels. ${ }^{31}$

The greatest number of experiments on double IR-IR resonance in MP excitation has been performed with the $\mathrm{SF}_{6}$ molecule. All these experiments can be conventionally divided into two types. The first type includes experiments on high-resolution spectroscopy of double resonance with tunable diode lasers. ${ }^{31-33}$ These experiments performed usually at low excitation levels are aimed mainly at studying the excitation mechanism of lower vibrational molecular levels and their spectroscopic parameters.

The second type unites experiments on double IR-IR resonance at rather high excitation levels. In these experiments consideration is given to the peculiarities of internal energy distribution under MP excitation, the quasicontinuum spectrum, intramolecular energy distribution, vibrational relaxation of highly excited molecules, etc.

Now we are going to consider in more detail some most important results obtained by the method of double IR-IR resonance.

Figure 5.10 shows the time evolution of the linear absorption spectrum of $\mathrm{SF}_{6}$ after MP excitation studied in work. ${ }^{34}$ It may be seen that right after an excitation pulse there are two maxima formed in the IR absorption spectrum which are transformed then to one cor:mon maximum. As shown in $^{34}$ by correlating the spectra produced with the spectra of $\mathrm{SF}_{6}$ under thermal heating in shock-wave tubes, ${ }^{44}$ the two maxima in the spectrum of $\mathrm{SF}_{6}$ under MP excitation correspond to the formation of two molecular ensembles: "cold" molecules remaining at the lower discrete vibrational levels and "hot" 


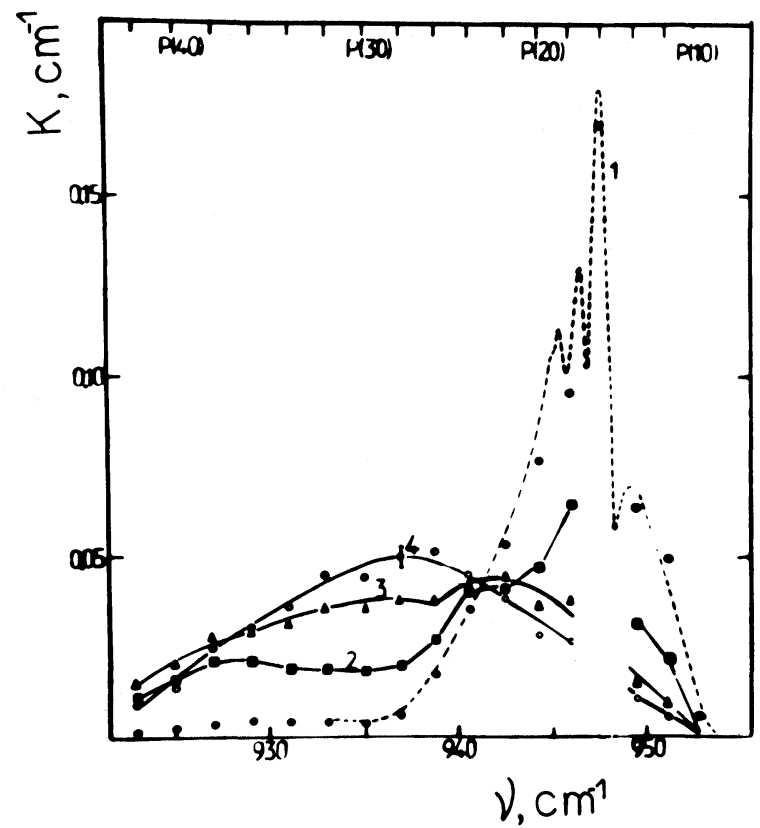

FIGURE 5.10 Evolution of $\mathrm{SF}_{6}$ absorption spectrum after MP excitation: $\Omega=$ $947.74 \mathrm{~cm}^{-1} ; \Phi=0.34 \mathrm{~J} / \mathrm{cm}^{2} ; p=0.18$ Torr, $\tau_{p}=10^{-7} \mathrm{~s} ; \tau_{d}$-moment of probing after the beginning of IR pulse. (1) Liner absorption spectrum, $1-\tau_{d}=0.2-\tau_{d}=100 \mathrm{~ns}$; $3-\tau_{d}=2.5 \mu \mathrm{s} ; 4-\tau_{d}=15 \mu \mathrm{s}$ (from Ref. 34 ).

molecules excited to high vibrational states. The shift of the absorption spectrum of vibrationally excited molecules towards the long-wave side which can be also easily observed under ordinary thermal heating of molecules ${ }^{44}$ is conditioned by vibration anharmonicity. The evolution of the absorption spectrum reflects the evolution of the system due to collisions of "hot" and "cold" molecules to vibrational distribution with one new vibrational temperature $T_{\text {vib}}$, which corresponds to the excess of vibrational energy $\bar{\varepsilon}$ absorbed by the molecules from the IR field. Cooling of $\mathrm{SF}_{6}$ causes the original absorption spectrum to be narrowed. So the double IR-IR resonance in cooled $\mathrm{SF}_{6}$ gas provides us ${ }^{45}$ with a more descriptive spectral picture of formation of two molecular ensembles.

In work ${ }^{38}$ the method of double IR-IR resonance is used to show that during IR photoexcitation of some polyatomic molecules, $\mathrm{SF}_{6}$ in particular, it is possible to perform purely radiative excitation of 
molecules from many rotational states. For example, when the $\mathrm{SF}_{6}$ molecule is excited at the frequency corresponding to the absorption line $P(63)$ the absorption at the $R(109)$ line decreases instantly without any collisions participating. This result as well as the results of measurement of double IR-IR resonance signals at other frequencies of exciting and probing IR radiation led to a conclusion drawn in Ref. 38 on the effect of depletion of many rotational states of the ground vibrational state in $\mathrm{SF}_{6}$. The existence of this effect was supported then in a number of experiments. ${ }^{34,46-49}$

In the above experiments carried out with the use of the method of double IR-IR resonance the second (probe) field was weak enough which provided the linearity of interaction of the probe field with vibrationally excited molecules. There may be another modification of the method of double IR-IR resonance when the second field is strong enough and can also perform MP excitation ${ }^{22}$ and even MP dissociation ${ }^{14}$ of molecules. Such two-frequency MP excitation of molecules is used with success in studying different aspects of MP processes and particularly in measuring the fraction of molecules involved in the process of MP excitation ${ }^{50}$ (see Section 5.3).

\subsection{2. $U V$ absorption (double IR-UV resonance)}

Vibrational excitation of molecules can bring about changes in their electronic absorption spectra ${ }^{51,52}$ and also in the characteristics of different processes related to UV absorption, such as UV predissociation, ${ }^{53} \mathrm{UV}$ dissuciation, ${ }^{52} \mathrm{UV}$ ionization, ${ }^{54} \mathrm{UV}$ isomerization, ${ }^{55} \mathrm{UV}$ luminescence. ${ }^{58}$ The recording of such changes can give important information on the process of MP excitation and on the subsequent relaxation processes of vibrationally excited molecules. ${ }^{51,56,57,11}$ Besides, the method of IR-MP excitation and subsequent electronic excitation is considered at present as one of the most promising techniques to carry out isotopically highly selective dissociation of molecules, especially in case of isotopes of heavy elements.

The method of IR-UV resonance was first applied in Ref. 56 to study the processes of vibrational excitation and $V-T$ relaxation of ammonia molecules. The vibrational excitation of the $\mathrm{NH}_{3}$ molecules under the action of IR $\mathrm{CO}_{2}$ laser pulses was recorded by new absorption lines in the discrete spectrum corresponding to the electronic transitions $\tilde{X} \rightarrow A^{\prime \prime}$ of $\mathrm{NH}_{3}$. 
The qualitative interpretation of this experiment was possible primarily due to the resolved structure of the UV spectrum of $\mathrm{NH}_{3}$. The line $\tilde{X}\left(v_{2}^{\prime \prime}=1\right) \rightarrow A^{\prime \prime}\left(v_{2}^{\prime}=0\right)$ is displaced towards the long-wave side about the spectrum of the electronic transition $\tilde{X}\left(v_{2}^{\prime \prime}=0\right) \rightarrow A^{\prime \prime}$, and the intensity of this line enables us to determine unambiguously the population of the vibrational level $v_{2}=1$ of the ground electronic state of $\mathrm{NH}_{3}$. The value obtained for $V-T$ relaxation of the $\nu_{2}$ vibration of $\mathrm{NH}_{3}$ was $p \tau_{V-T}=2.4 \pm 0.4 \mu \mathrm{s}$ Torr. As these experiments show, there was no effect of MP excitation in $\mathrm{NH}_{3}$ with the laser pulse energy fluence $\Phi \leqslant 2 \mathrm{~J} / \mathrm{cm}^{2}$. It should be noted that the process of laser isotope separation was first realized with IR-UV excitation of $\mathrm{NH}_{3} .{ }^{53}$

Studies on UV absorption spectra at MP excitation have been carried out for the molecules $\mathrm{OsO}_{4},{ }^{51} \mathrm{CF}_{3} \mathrm{I}^{52} \mathrm{C}_{2} \mathrm{H}_{2} \mathrm{Cl}_{2} .{ }^{55}$ etc. Figure 5.11 shows the change of the $\mathrm{UV}$ absorption spectrum of $\mathrm{CF}_{3} \mathrm{I}$ caused by the excitation of its high vibrational levels. In Ref. 52 quantitative interpretation of the results obtained was realized. Consideration is given there to the model which allows for the fact that just a fraction of molecules $(q)$ is excited to high vibrational states. Initially the IR radiation excites the vibrational mode $\nu_{1}$, and UV absorption must change under vibrational excitation of just the mode $\nu_{3}$ corresponding to the bond $\mathrm{C}-\mathrm{I}$. Therefore, full stochastization of vibrational energy in the molecule (see Ref. 1, Section 2.3) was taken to explain the UV absorption variation observed in Ref. 52. The model of Boltzmann distribution of vibrational levels populations in the "hot" molecular ensemble described satisfactorily the experimental data.

It must be also said that research of the time evolution of double IR-UV resonance spectra allows direct measuring of the rate of vibrational energy exchange between excited and unexcited molecules. In ref. 57 for example, the rate constant of $V-V^{\prime}$ exchange between the $\mathrm{CF}_{3} \mathrm{I}$ molecule with an average vibrational energy $E-6300 \mathrm{~cm}^{-1}$ and the unexcited molecules was

$$
K=2 \mu \mathrm{s}^{-1} \cdot \operatorname{Torr}^{-1} \text {. }
$$

Experiments on MP excitation of molecules in molecular beams necessitate the elaboration of methods of spectroscopic diagnostics which can be applied under low molecular concentrations. One of slich methods is UV photoionization of molecules after IR multiphoton excitation in a molecular beam. In ref. 54 this method was used to 


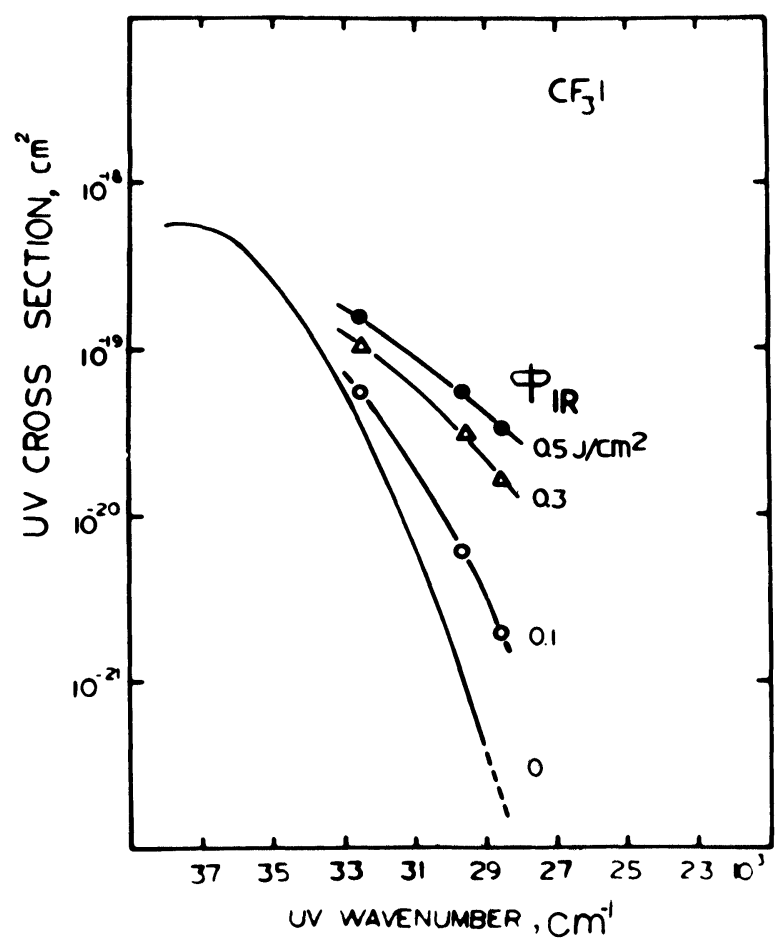

FIGURE 5.11 Spectra of UV absorption of MP excited $\mathrm{CF}_{3} \mathrm{I}$ molecules: $p_{\mathrm{CF}_{3} \mathrm{I}}=$ 0.5 Torr. (1) $\Phi=0 ;$ (2) $\Phi=0.1 \mathrm{~J} / \mathrm{cm}^{2}$; (3) $\Phi=0.3 \mathrm{~J} / \mathrm{cm}^{2}$; (4) $\Phi=0.5 \mathrm{~J} / \mathrm{cm}^{2}$ (from Ref. 52).

determine the properties of vibrational energy distribution of $\mathrm{SF}_{6}$ molecules with the energy fluences being below the dissociation threshold. Figure 5.12 shows the photoionization spectra of $\mathrm{SF}_{6}$ molecules in a beam at several different radiation energy fluences. With an increase in $\Phi$ we can observe a gradual displacement of the photoionization band edge towards the long-wave side which is identical to the displacement of UV absorption bands (see, for example, Figure 5.10) These spectra, however, cannot give quantitative information on the vibrational distribution function. The qualitative estimates obtained from correlating the photoionization spectra of $\mathrm{SF}_{6}$ at multiple photon excitation with the spectra of heated $\mathrm{SF}_{6}$ molecules made it possible to conclude in Ref. 54 that, even though a large fraction 


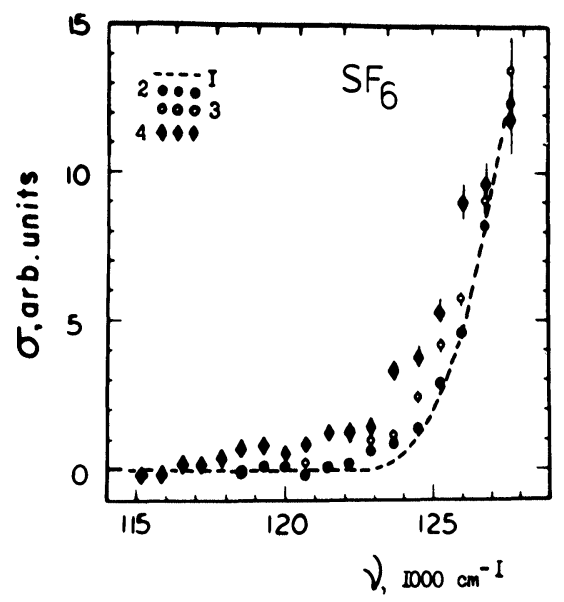

FIGURE 5.12 Frequency dependences of $\mathrm{SF}_{6}$ photoionization cross-section in molecular beam: $\Omega=942.38 \mathrm{~cm}^{-1} ; T=300 \mathrm{~K}$. (1) $\Phi=0$; (2) $\Phi=0.2 \mathrm{~J} / \mathrm{cm}^{2}$; (3) $\Phi=$ $0.4 \mathrm{~J} / \mathrm{cm}^{2}$; (4) $\Phi=1.5 \mathrm{~J} / \mathrm{cm}^{2}$ (from Ref. 54 ).

of molecules in a beam at moderate excitation levels interacts with the field, most of these molecules absorb energy that is insufficient to excite them to the quasi-continuum (about 3 photons, on the average) and remain at discrete levels.

\subsubsection{Raman scattering (double IR-RS resonance)}

The methods of Raman scattering (RS) spectroscopy (see Ref. 1, Section 2.2.5) began to be used just a short time ago to study MP excitation of molecules by IR radiation. This became possible due to advances in the experimental technique of RS spectroscopy in gases and particularly in the technique of RS with laser excitation.

Spontaneous Raman scattering The most important merit of spontaneous RS diagnostics is that it is possible to separate direct (Stokes RS) and reverse (anti-Stokes RS) transitions in the spectrum. In this case, the integrated intensity of RS in the anti-Stokes region is proportional to the vibrational energy $\bar{\varepsilon}_{\nu_{\mathrm{RS}}}$ stored in the mode $\nu_{\mathrm{RS}}$ :

$$
S_{\nu_{\mathrm{RS}}^{\mathrm{AS}}}^{\mathrm{A}} \sim \bar{\varepsilon}_{\nu_{\mathrm{RS}}}
$$


(see Ref. 1, Section 2.7). This simple result has been obtained experimentally in Ref. 60 (Figure 5.13) and has a number of important consequences. For example, by measuring the integral intensities of RS signals corresponding to different modes we can speak about intramolecular distribution of vibrational energy ${ }^{59,63}$ (see Chapter 6.).

Studies on the RS spectra both in the Stokes and anti-Stokes regions with rather high spectral resolution allows obtaining quantitative information on the function of vibrational energy distribution in the process of MP excitation. ${ }^{61} \mathrm{We}$ shall illustrate this fact on the example of $\mathrm{SF}_{6}$. Spectra of anti-Stokes Raman signal for $\mathrm{SF}_{6}$ have been presented

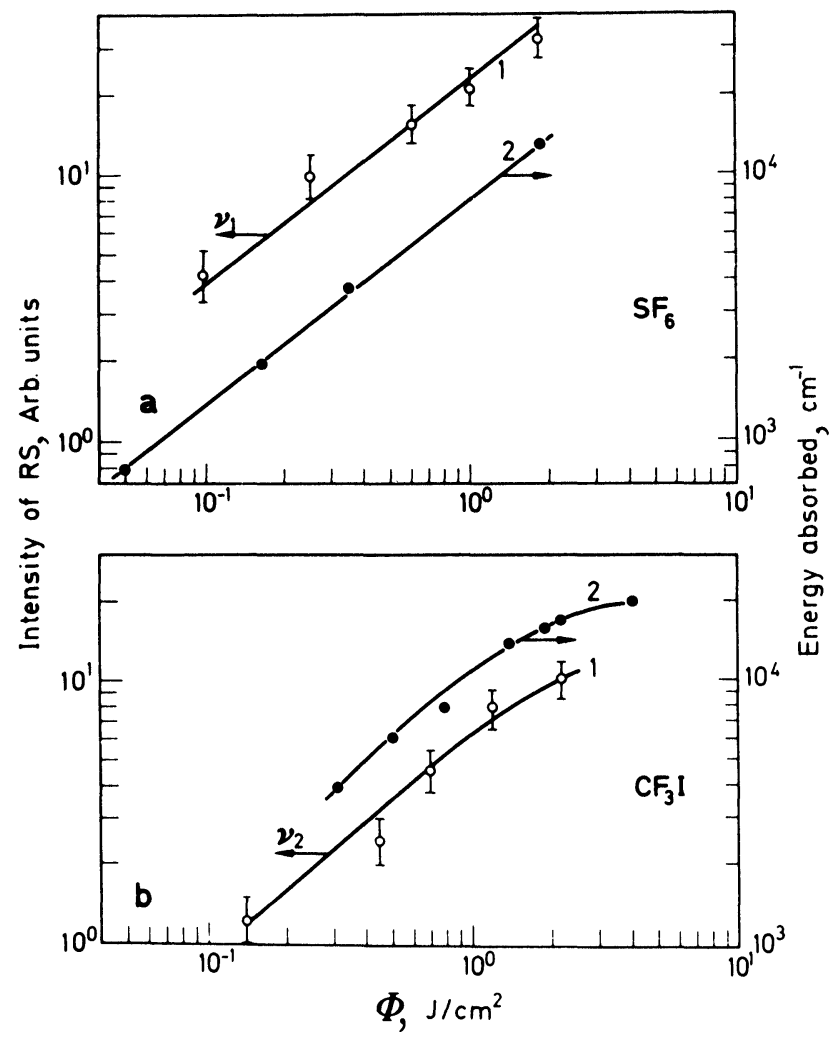

FIGURE 5.13 Dependences of integral intensity of anti-Stokes Raman signal $S^{\text {AS }}$ and average absorbed energy $\bar{\varepsilon}$ on laser energy fluence $\Phi$ : (a) $\mathrm{SF}_{6}$ molecule; (1) $S^{\mathrm{AS}}(\Phi)$; (2) $\bar{\varepsilon}(\Phi)$; (b) $\mathrm{CF}_{3} \mathrm{I}$ molecule; (1) $S^{\mathrm{AS}}(\Phi),(2) \bar{\varepsilon}(\Phi)$ (from Ref. 50 ). 
in Ref. 2 (Figure 4.2). In the absence of the $\mathrm{CO}_{2}$ laser pulse there is one peak observed with its center at $775 \mathrm{~cm}^{-1}$ corresponding to the $\nu_{3}$ mode. The peak width is determined by spectral resolution which in Ref. 61 comes to $10 \mathrm{~cm}^{-1}$. Irradiation of $\mathrm{SF}_{6}$ by $\mathrm{CO}_{2}$ laser pulses materially changes the RS spectrum. There are two maxima in the spectrum which can be interpreted, like in case of double IR-IR resonance (Figure 5.10), as the formation of "cold" and "hot" molecular ensembles. If the delay time between the IR (exciting) and UV (probing) pulses is increased to $3 \mu$ s there will be only one maximum in the RS spectrum which stems from $\mathrm{V}-\mathrm{V}$ exchange, as a result of which two ensembles finally convert to one.

The collisions of the molecules with the buffer gas during an IR pulse also cause the form of vibrational distribution function to change. The signal from the excited molecules in this case rises and that from the unexcited molecules drops. At rather a high pressure of buffer gas the undisplaced peak disappears completely. In this case the RS spectrum of excited molecules does not shift in the presence of buffer gases. It means that under the conditions of the experiment the collisions with the buffer gas $(p \tau=6 \mu \mathrm{s} \cdot$ Torr $)$ do not bring about appreciable changes of average energy in the "hot" ensemble of molecules. This suggests an important conclusion that rotational relaxation involving new $\mathrm{SF}_{6}$ molecules from initial quantum states into the process of MP excitation has no pronounced effect on their subsequent excitation by the IR field.

It should be noted that the method of double IR-RS resonance can be used in studying both the processes of MP excitation and subsequent relaxation processes of molecules in quasicontinuum.

Coherent anti-Stokes Raman scattering CARS spectroscopy of molecular gases (see Ref. 62) is now widely used to get new spectroscopic information and for analytical purposes. A principal advantage of CARS with respect to spontaneous RS is a high spectral resolution, which is determined by spectral width of laser sources, used in CARS spectrometer. For this reason CARS spectroscopy is highly perspective for studying of MP excitation at lower vibrational levels. At the same time, applications of CARS for studying of MP excitation in quasicontinuum is quite difficult, since CARS intensity is inversely proportional to the absorption linewidth in the second power. Besides, CARS signal is proportional to the difference of populations of vibrational levels 
(but not to the population, as in the case of spontaneous RS), and quantitative interpretation of CARS, spectra is more complicated with respect to the spontaneous Raman spectra.

It should be pointed out that CARS probing is quite similar to the high resolution double IR-IR resonance (see Section 5.2.1).

CARS diagnostics of MP excited $\mathrm{SF}_{6}$ molecules is used in Refs. 63-64. Figure 5.14 shows CARS spectra of $\mathrm{SF}_{6} \nu_{1}$ mode at various time delays $\tau_{d}$ between exciting and probing pulses. Changing of CARS spectrum reflects the temporal evolution of vibrational energy distribution due to the collisional energy exchange.

\subsubsection{IR fluorescence}

Observation of spontaneous IR fluorescence in MP excitation of molecules can also yield essential information on the process of MP excitation.

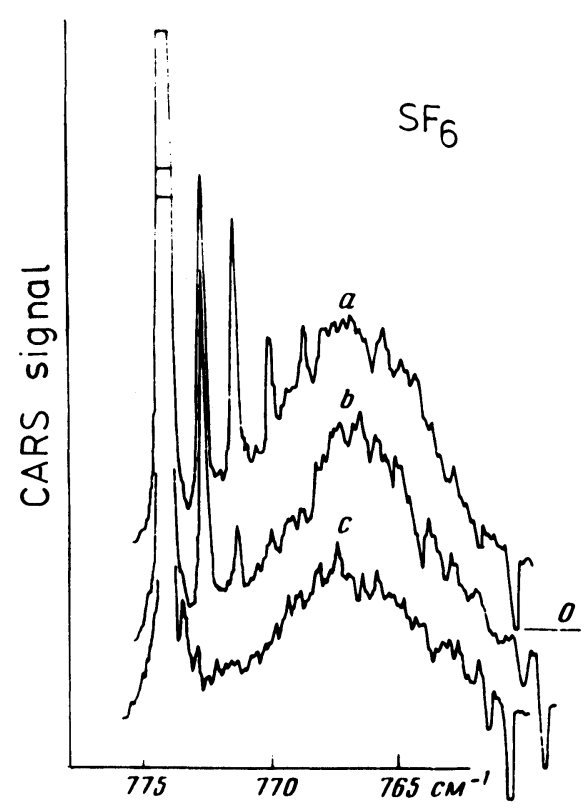

FIGURE 5.14 CARS spectra for $\mathrm{SF}_{6}$ molecule $\nu_{1}$ mode at various delays of time $\tau_{d}$ between exciting and probing pulses: (a) $\tau_{d}=100 \mathrm{~ns}$; (b) $\tau_{d}=200 \mathrm{~ns}$; (c) $\tau_{d}=2 \mu \mathrm{s}$. $\Phi=0.2 \mathrm{~J} / \mathrm{cm}^{2}$ (from Ref. 64). 
In this connection we are going to consider first of all the results of experiments on IR fluorescence of $\mathrm{SF}_{6}$ under ordinary thermal heating. ${ }^{56}$ The measurements with $T=1780 \mathrm{~K}$ show that the fluorescence spectrum maximum is displaced by $40 \mathrm{~cm}^{-1}$ towards the longwave side from the harmonic position of the $\nu_{3}$ mode, and the fluorescence band width, with allowance made for self-absorption in gas, equals $42 \pm 6 \mathrm{~cm}^{-1}$. At such high temperatures the average vibrational energy of $\mathrm{SF}_{6}$ molecules will be $\bar{\varepsilon}=15000 \mathrm{~cm}^{-1}$. In this case most of the molecules are already in the quasicontinuum but, nevertheless, their IR fluorescence spectrum is not continuous but localized near the harmonic position of the $\nu_{3}$ mode.

Much the same behavior of the IR fluorescence spectra of $\mathrm{SF}_{6}$ is observed in MP excitation of the molecule. ${ }^{56}$ With an increase of the value of absorbed energy $\bar{\varepsilon}$ in this case there is a linear shift of the maximum towards the long-wave side and a broadening of the IR fluorescence spectrum band which is related to vibration anharmonicity and an increase in the vibrational distribution width.

Figure 5.15 shows spectral dependences of IR fluorescence in $\mathrm{SF}_{6}$ measured in the case of MP excitation and in the case of thermal heating. Two maxima are clearly observed in the case of MP excited molecules, corresponding to the two molecular ensembles. The fact of existing of short-wave maximum means itself that some fraction of excited $\mathrm{SF}_{6}$ molecules remains at discrete low-lying vibrational levels.

IR fluorescence in the $\mathrm{SF}_{6}$ molecule during its $\mathrm{MP}$ excitation by $\mathrm{CO}_{2}$ laser radiation has been also studied in a spectral region of $16 \mu \mathrm{m}$ where the $\nu_{4}$ vibrational mode lies. ${ }^{67}$ It has been found that the time of IR fluorescence build-up is much shorter than the times of any collisional processes in gas. On the basis of this fact a conclusion has been drawn in Ref. 67 on excitation of $\mathrm{SF}_{6}$ molecules to quantum states possessing the properties of vibrational quasicontinuum. In other words, qualitative observation of vibrational energy stochastization at multiphoton excitation is possible. The spectral-integrated intensity of IR fluorescence $S_{\nu_{\mathrm{IR}}}^{\text {fuuor }}$ corresponding to the $\nu_{\mathrm{IR}}$ mode, similarly to relation (5.5), is proportional to the vibrational energy $\bar{\varepsilon}_{\nu_{\mathrm{IR}}}$ stored in the $\nu_{\mathrm{IR}}$ mode (see Ref. 1, Section 2.7):

$$
S_{\nu_{\mathrm{IR}}}^{\text {fluor }} \sim \bar{\varepsilon}_{\nu_{\mathrm{IR}}}
$$

So, by measuring the integral intensities of IR fluorescence corresponding to different modes of the molecule we can estimate its 

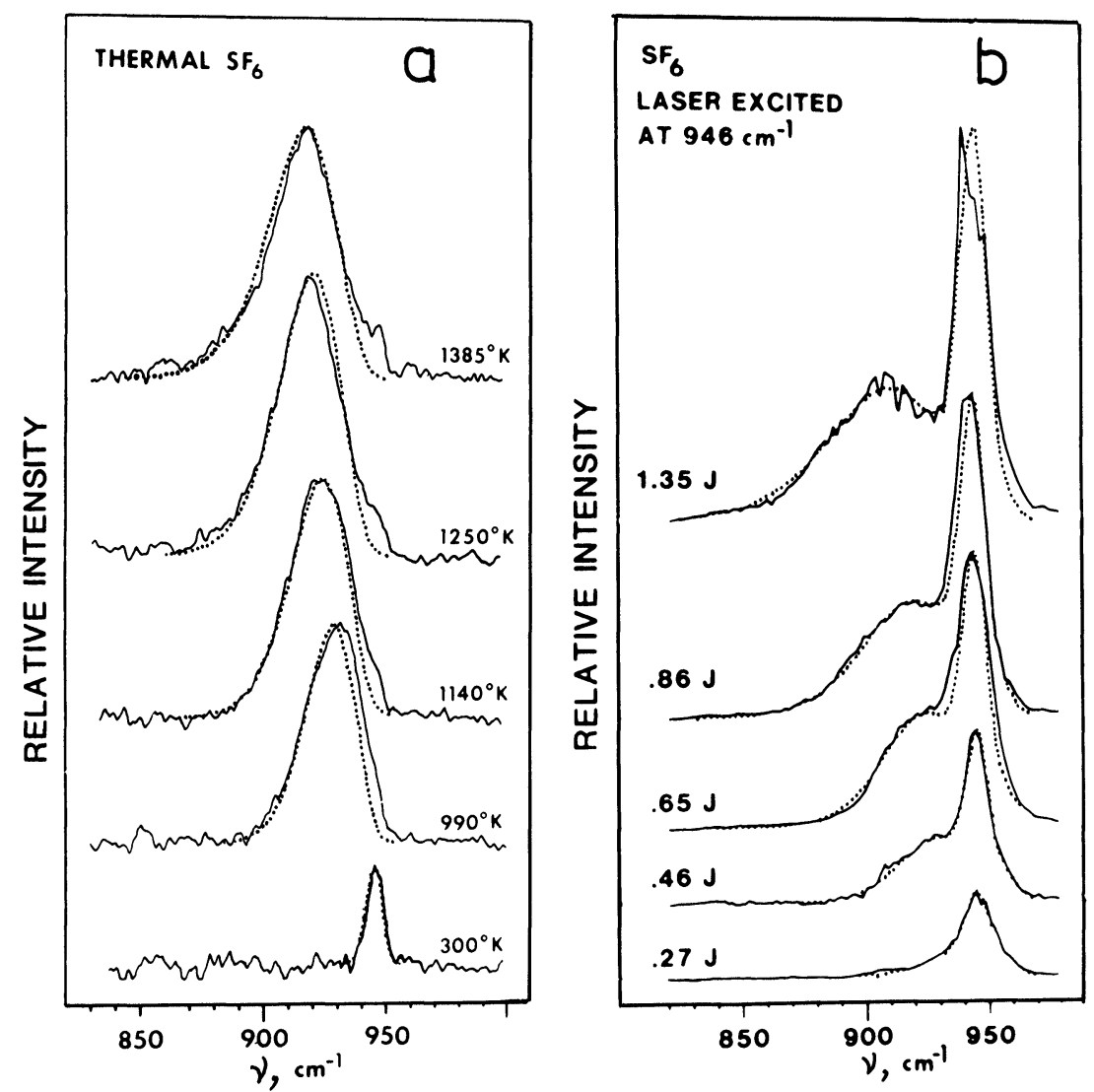

FIGURE 5.15 Spectra of IR fluorescence for $\mathrm{SF}_{6}$ molecule (a) thermal excitation; (b) MP excitation (from Ref. 66).

intramolecular energy distribution. In Ref. 68 , for example, the energy distribution over different modes for the $\mathrm{CH}_{3} \mathrm{~F}$ molecules was studied in the process of collisions during its vibrational excitation by $\mathrm{CO}_{2}$ laser radiation. The laser pulse energy fluence in ref. 68 was insufficient for collisionless MP excitation of $\mathrm{CH}_{3} \mathrm{~F}$. But the technique suggested in Ref. 68 is quite suitable to determine the intramolecular distribution of vibrational energy under MP excitation as well as to study some other parameters of the process, the fraction of molecules $q$, for example, involved in the process of MP excitation. 


\subsection{Fraction of molecules involved in multiple photon excitation}

The fraction of molecules $q$ involved in the process of MP excitation is one of the fundamental questions when the passage of molecules through lower vibrational levels is under consideration. In this section we are going first to discuss the available methods used to measure the $q$ value: the methods based on the inclusion of the influence of collisions on the characteristics of MP processes and the methods based on spectroscopic diagnostics of molecules under MP excitation. Then, in Section 5.3.3 we shall present the known results of measurements of the $q$ value for a number of molecules.

\subsubsection{Role of collisions in passage of lower vibrational levels}

Since the interaction of the molecule with the IR field depends greatly on its initial vibrational-rotational state the collisions which change the molecular state for the IR radiation pulse time $\tau_{p}$ must affect the process of MP excitation. There are several types of collisional relaxation processes in gases (Section 2.5 in Ref. 1). The characteristic cross-sections of these processes depend materially on the type of collisional partners as well as on their initial and final quantum states. The fastest collisional process is rotational relaxation with its crosssection being of the order of gas-kinetic one. It changes the energy of a molecule little, but can significatitly change its interaction with the IR field. This influence is most essential in the region of lower vibrational levels. Other types of collisional relaxation processes- $V-$ $V^{\prime}$-exchange and $V-T, R$-relaxation-have cross-sections which are smaller than gas-kinetic ones. Some influence of these processes on the character of excitation of lower molecular levels manifests itself only at rather high gas pressures.

In Ref. 2 (Section 3.3) a rather simple model for excitation of an ensemble of two-level systems distributed over different rotational states with rotational relaxation in the ground and excited vibrational states have been considered. This model can be apparently realized only in case of IR excitation of sufficiently simple molecules in moderate IR fields when the excitation of vibrational levels with $v>1$ may be neglected. But it is descriptive enough and enables qualitative understanding of the role of rotational relaxation in case of MP excitation of the lower levels of polyatomic molecules, too. 
Let us consider now for the two-level model the dependence of the number of absorbed quanta $\bar{n}$ on $\tau_{p} / \tau_{\text {rot }}$ that is the ratio of pulse duration to rotational relaxation time. It is also assumed that the laser pulse energy fluence $\Phi$ is larger than the energy fluence of vibrational transition saturation $\Phi_{s}\left(\Phi_{s}=\hbar \Omega / 2 \sigma\right)$. In this case, according to ref. 69 , we get the following approximated expression

$$
\bar{n}=\frac{1}{2}\left[1-(1-f) \exp \left(-f \tau_{p} / \tau_{\text {rot }}\right)\right]
$$

where $f$ is the relative population of the rotational states resonant with the IR field. The character of the dependence of the number of absorbed quanta $\bar{n}$ on the value of $\tau_{p} / \tau_{\text {rot }}$ is fully determined by the $f$ value. The maximum increase of $\bar{n}$, when passing from collisionless $\left(\tau_{\text {rot }} \gg \tau_{p}\right)$ to collisional excitation $\left(\tau_{\text {rot }} \ll f \tau_{p}\right)$, will be

$$
\bar{n}_{\text {collisionless }} / \bar{n}_{\text {collisional }}^{\text {max }}=f
$$

The experiments carried out with simple molecules confirm the validity of the two-level model in describing the effect of collisions on absorption saturation. For quantitative description we should know the value of $f$. Having identification of the lines in the absorption spectrum of the molecule and knowing the spectral composition of radiation, we can, in principle, determine this value theoretically. In practice, however, this is rather difficult, particularly in case of molecules with a complex vibrational-rotational spectrum. An approximation for the $q$ value of such molecules is given in ref. 2 by expression (3.78).

The $q$ value can be estimated more accurately experimentally on the basis of the two-level model by studying the saturation of IR radiation absorption in two limiting cases: at a low gas pressure when $\tau_{\text {rot }} \ll \tau_{p}$ and at rather a high pressure of (absorbing or buffer) gas when deliberately $\tau_{\text {rot }} \ll f \tau_{p}$. The $f$ value in this case can be found from (5.8). It depends to a large extent on the type of the molecule being excited and on the properties of IR radiation (intensity $I$, frequency $\Omega$, spectral line width $\delta \Omega$ ). For example, if the molecule is excited by IR radiation pulses of a standard TEA $\mathrm{CO}_{2}$ laser with $\Phi \approx \hbar \Omega / \sigma_{0}$ and $\tau \approx 10^{-7} \mathrm{~s}$, the $f$ factor, according to all estimations, is no higher than $10^{-2}-10^{-3}$.

The description of the process of IR excitation using the two-level model holds true only for rather simple molecules in moderateintensity fields. In case of more complex molecules, even at moderate 
intensities one can observe MP absorption and a corresponding deviation from the two-level model behavior. MP absorption involves the molecules from those initial vibrational-rotational states for which nultiphoton and multistep resonances at the field frequency are possible. The spectrum of these resonances is, as a rule, almost unknown. So it is rather difficult to determine theoretically the population of the rotational states participating in MP absorption. Besides, the cross-sections of excitation of molecules from different initial states at the field frequency $\Omega$ may differ very materially. Therefore, in case of MP absorption it is advisable to average the description of the process of molecular excitation at lower levels with the use of the $q$ factor without specifying the initial vibrational-rotational states at which excitation occurs (see ref. 2, Section 4.2.3). Physically this description is based on experimentally observed division of molecules into two ensembles: "cold" molecules not interacting with the field and "hot" molecules excited to high vibrational states.

It is much more difficult to describe quantitatively MP excitation during collisions than for the two-level model since, generally speaking, rotational relaxation should be considered in initial, final and intermediate vibrational states. The qualitative role of rotational relaxation in MP excitation is similar to that in the two-level model. It leads to population of the rotational states for which multiphoton and multistep resonances are possible and thereby increases the fraction of molecules involved in the process of MP excitation. The influence of rotational relaxation on the passage of lower levels can be understood from the dependence of different characteristics of MP processes, such as average absorbed energy and dissociation yield on absorbing or buffer gas pressures. Below we consider these dependences for some molecules.

The effect of collisions on the characteristics of MP processes has been studied in detail for $\mathrm{SF}_{6}$ molecule. Figure 5.16 shows the dependence of the average number of absorbed quanta $\bar{n}$ on the pressure of $\mathrm{SF}_{6}$ molecules over the range from 0.2 to 4 Torr for different values of laser energy fluence $\Phi$ (from Ref. 25). It may be seen that the dependence of $\bar{n}$ on $p$ varies with $\Phi$ substantially and reaches its maximum over the range $\Phi=10^{-2}-10^{-1} \mathrm{~J} / \mathrm{cm}^{2}$. With further increase in $\Phi$ the rate of increase of $\bar{n}$ drops. It should be also noted that the dependence of $\bar{n}$ on $p$ with $\Phi=0.052 \mathrm{~J} / \mathrm{cm}^{2}$ has the form $\bar{n} \sim p^{1.6}$ even at rather low gas pressures $p=0.2$ Torr. 


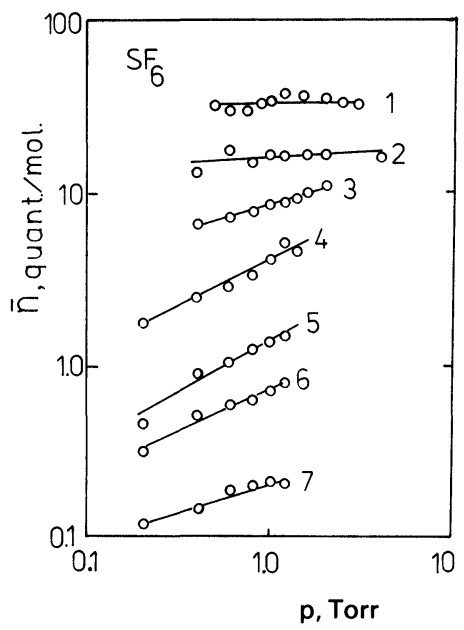

FIGURE 5.16 Dependences of average number of absorbed quanta on $\mathrm{SF}_{6}$ pressure- $p$ (direct calorimetry) $\left(\tau_{p}=50 \mathrm{~ns} ; \Omega=940.55 \mathrm{~cm}^{-1}\right) ;(1) \Phi=1.3 \mathrm{~J} / \mathrm{cm}^{2}$; (2) $\Phi=0.40 \mathrm{~J} / \mathrm{cm}^{2}$; (3) $\Phi=0.18 \mathrm{~J} / \mathrm{cm}^{2}$; (4) $\Phi=0.052 \mathrm{~J} / \mathrm{cm}^{2}$; (5) $\Phi=0.016 \mathrm{~J} / \mathrm{cm}^{2}$; (6) $\Phi=0.0077 \mathrm{~J} / \mathrm{cm}^{2}$; (7) $\Phi=0.0019 \mathrm{~J} / \mathrm{cm}^{2}$ (from Ref. 25).

Molecular photoexcitation is strictly collisionless if the quantum state of the molecule in the process of excitation changes only under the action of radiation field. In other words, the time of molecule-field interaction must be much shorter than the characteristic time of any collisional processes, the time of rotational relaxation in particular. With the pulse duration $\tau_{p}=10^{-7} \mathrm{~s}$ the collisionless conditions of excitation can be evidently realized at gas pressures $<10^{-3} \cdots 10^{-4}$ Torr. At the same time most of the studies in MP absorption have been carried out at present at pressures no lower than 0.1 to 0.5 Torr and $\tau_{p}=10^{-7}-10^{-6} \mathrm{~s}$ when the influence of rotational relaxation during an IR pulse is not eliminated completely.

The method of opto-thermal calorimetry of absorbed energy ${ }^{16}$ (see 5.1.1) at very low pressures has allowed distinct following of the changes in MP absorption in passing from collisionless to collisional excitation. Figure 5.17 presents some dependences of the $\bar{\varepsilon}$ value (in relative units) on the pressure of $\mathrm{SF}_{6}$ gas at several values of $\Phi$ (the excitation conditions are given in the legend for Figure 5.17). In the region of low pressures $p<2 \cdots 5 \cdot 10^{-3}$ Torr the value of $\bar{\varepsilon}$ is independent on $p$, which testifies to the collisionless nature of absorption. As 


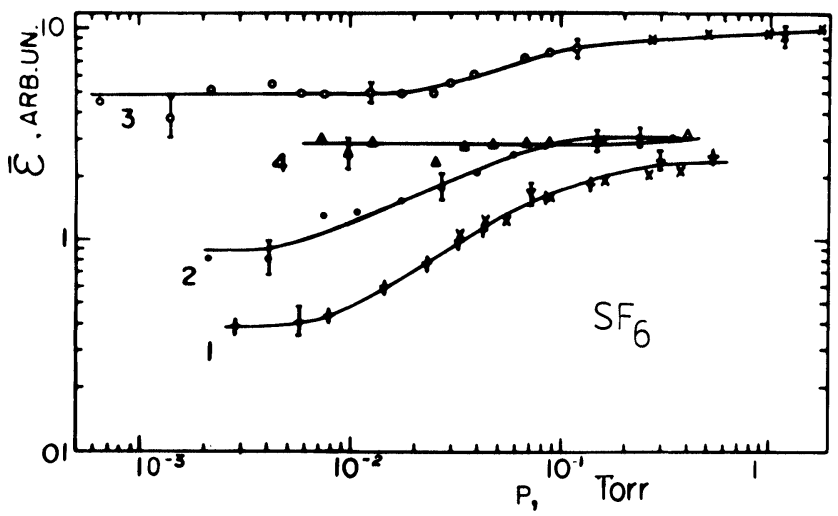

FIGURE 5.17 Dependences of average absorbed energy $\bar{\varepsilon}$ on $\mathrm{SF}_{6}$ pressure $p$ (optothermal calorimetry): (1) $\Phi=0.042 \mathrm{~J} / \mathrm{cm}^{2}$; (2) $\Phi=0.11 \mathrm{~J} / \mathrm{cm}^{2}$; (3) $\Phi=0.35 \mathrm{~J} / \mathrm{cm}^{2}$; (4) $\Phi=0.07 \mathrm{~J} / \mathrm{cm}^{2}\left(\tau_{p}=50 \mathrm{~ns}, \Omega=947.74 \mathrm{~cm}^{-1}\right)$ (from Ref. 16).

$p$ increases one can observe an increase in $\bar{\varepsilon}$ due to the influence of rotational relaxation. Then saturation occurs which is related to the involvement of all the $\mathrm{SF}_{6}$ molecules into the process of excitation.

Dependences similar to these ones in qualitative respect can be observed when a buffer gas is added to $\mathrm{SF}_{6}$. As the pressure of the buffer gas $p_{\text {buff }}$ (for example, $\mathrm{N}_{2}$ or $\mathrm{Xe}^{70}$ ) increases, the value of $\bar{\varepsilon}$ grows and then comes to saturation. Besides, according to ref. 70, with increasing the maximum relative increase of $\bar{\varepsilon}$ drops, and with $2.2-2.3 \mathrm{~J} / \mathrm{cm}^{2}$ the value of $\bar{\varepsilon}$ does not depend on $p_{\text {buff }}$ at all.

Let us consider here the effect of rotational relaxation on the characteristics of MP dissociation of the $\mathrm{SF}_{6}$ molecule. Effective MP dissociation of $\mathrm{SF}_{6}$ is observed when the $\mathrm{CO}_{2}$ laser energy fluence $\Phi>2 \mathrm{~J} / \mathrm{cm}^{2}$. Under these conditions, according to ref. 70 , rotational relaxation almost does not bring about an increase in absorbed energy. Consequently, the MP dissociation yield $\beta$ must not increase either as the pressure of $\mathrm{SF}_{6}$ or the buffer gas increases. This is supported by the results of direct experiments. In Ref. 13, for example, it has been shown that with a rise in pressure of buffer gas $\left(\mathrm{Ar}, \mathrm{O}_{2}, \mathrm{C}_{2} \mathrm{H}_{4}\right.$ or $\mathrm{NH}_{3}$ ) in the pressure range under study (1-100 Torr) one can always observe only a monotonous exponential decrease of the $\beta$ value with increasing buffer gas pressure, which is quite naturally explained by vibrational deactivation of $\mathrm{SF}_{6}$ due to $V-V$ and $V-T$, $R$ processes. 
The observed dependences of absorbed energy and dissociation yield on absorbed pressure and buffer gas pressure vary greatly. They are governed by the type of the molecule, the conditions of its excitation and the type of the buffer gas. When the radiation interacts with a considerable fraction of molecules $q$, rotational relaxation must not lead to a substantial increase of absorbed energy or dissociation yield (see Figure 5.18). On the contrary in the case of $q \ll 1$ rotational relaxation must lead to the substantional increase of $\bar{\varepsilon}$ and $\beta$ values.

The growth of dissociation yield with an addition of buffer gas is related to the effect of rotational "bottle neck" (see Ref. 2, Section 3.3). The increase of the rotational relaxation rate during a laser pulse causes the rotational states interacting with the radiation to be populated. As a result, the number of molecules excited by the radiation and, probably, the rate of excitation, as they pass through lower vibrational levels, are increased. The approximate calculations carried out on the basis of such an idea of rotational relaxation effect ${ }^{72,73}$ are in good agreement with the experimental dependences of dissociation yield on pressure. The comparison of model with experiment, ${ }^{72}$ for example, results in $p \tau_{\text {rot }}=160 \mathrm{~ns} \cdot$ Torr for the rotational relaxation time in the mixture $\mathrm{C}_{2} \mathrm{H}_{4}: \mathrm{Xe}(1: 40)$. This value is typical for rotational relaxation in collisions with the buffer gas. In case of collisions between $\mathrm{C}_{2} \mathrm{H}_{4}$ molecules value obtained for $p \tau_{\text {rot }}$ was $15-20 \mathrm{~ns} \cdot$ Torr.

As it is experimentally shown a change-over from collisionless to the collisional regime of excitation does not lead to the substantional

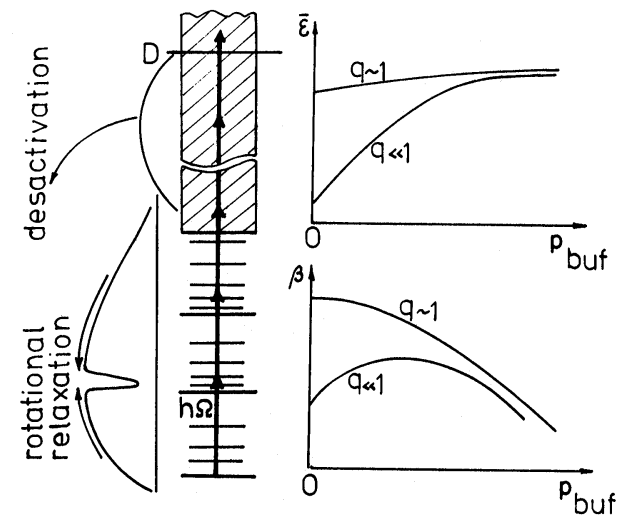

FIGURE 5.18 Effect of rotational relaxation and vibrational deactivation on absorbed energy $\bar{\varepsilon}$ and dissociation yield $\beta$. 
increase of absorbed energy or dissociation yield for heavy and large molecules. At the same time for light few-atom molecules such as $\mathrm{C}_{2} \mathrm{H}_{4}, \mathrm{CH}_{3} \mathrm{OH}, \mathrm{CF}_{2} \mathrm{HCl}, \mathrm{CDF}_{3}$ a significant increase of $\bar{\varepsilon}$ and $\beta$ values is observed. For $\mathrm{CDF}_{3}$ molecule, for example, an increase of $\mathrm{Ar}$ pressure from 0 to 20 Torr leads to the 45 -fold increase of $\beta$ value. $^{71}$

The fraction of molecules interacting with the field may depend on laser radiation fluence. This is supported by the experiments with $\mathrm{SF}_{6}$, $\mathrm{O}_{5} \mathrm{O}_{4}, \mathrm{CF}_{3} \mathrm{I}$ and other molecules (see Section 5.4). Therefore the contribution of rotational relaxation may also depend on the $\Phi$ value. In MP dissociation of the $\mathrm{C}_{2} \mathrm{H}_{3} \mathrm{~F}$ molecule, for example, with 11 Torr of $\mathrm{He}$ added, the relative increase of dissociation yield gradually decreases from 6.5 to 3.2 times as the $\Phi$ value varies from 3 to $45 \mathrm{~J} / \mathrm{cm}^{2}{ }^{78}$

The variation of the laser radiation frequency $\Omega$ may also affect essentially the role of rotational relaxation. The detuning from the maximum of MP absorption spectral band results, as a rule, in a decrease of the fraction of molecules involved into the process of excitation ${ }^{16}$ and hence in an increase of the contribution of rotational relaxation to $\mathrm{MP}$ absorption and dissociation. In case of the $\mathrm{CF}_{3} \mathrm{Br}$ molecule, for example, if the relative increase of dissociation yield at the center of the band is 3.5 times, it comes to 13 times in case of detuning to the far edge of the band $\left(18 \mathrm{~cm}^{-1}\right.$ towards the short-wave side from the center). ${ }^{75}$

It should be noted that it is rather important to perform strict control over the conditions of excitations for proper interpretation of the results of MP excitation. One of the potential causes of errors in interpretation, for example, may be noncorrective allowance for the pulse "tail" of $\mathrm{CO}_{2}$ laser pulse. The spectral width of radiation line, which for the ordinary atmospheric-pressure $\mathrm{CO}_{2}$ laser equals $\delta \Omega=-$ 0.01 to $0.03 \mathrm{~cm}^{-1}$, plays a no less important part especially in excitation of lower vibrational levels and in MP excitation of molecules with a rare vibrational-rotational spectrum. The $\delta \Omega$ value is at least one order higher than the Doppler absorption line width of molecules at room temperature, and IR pulse nonmonochromaticity can be neglected only provided that the power broadening value $\gamma>\delta \Omega$. In case of $\mathrm{SF}_{6}$ molecules, for instance, this corresponds to the radiation intensity $I>10^{5} \mathrm{~W} / \mathrm{cm}^{2}$ when MP absorption begins to play an essential part. 


\subsubsection{Determination of the $q$ value}

Method of collisions As shown in Ref. 61, the average excitation level in quasicontinuum for the $\mathrm{SF}_{6}$ molecule is independent on pressure. This fact allows the value of the $q$ factor to be determined approximately from the dependence of absorbed energy $\bar{\varepsilon}$ and dissociation yield $\beta$ on absorbed gas or buffer gas pressure

$$
\begin{aligned}
& q=\bar{\varepsilon} / \bar{\varepsilon}_{\max } \\
& q=\beta / \beta_{\max }
\end{aligned}
$$

In expressions (5.9) and (5.10) the values $\bar{\varepsilon}$ and $\beta$ are related to the initial gas pressure at which the $q$ value is determined. The values $\bar{\varepsilon}_{\max }$ and $\beta_{\max }$ correspond to maxima of the dependences $\bar{\varepsilon}(p)$ and $\beta(p)$ when it is assumed that $q=1$.

Yet there are two limitations here. First, with addition of a large amount of buffer gas the process of vibrational deactivation may become essential, when the buffer is molecular gas in particular (see Figure 5.18). Therefore the above described determination of the $q$ value will be rigorous enough provided that

$$
\tau_{\text {rot }} / q \ll \tau_{v}
$$

where $\tau_{\text {rot }}$ is the rotational relaxation time, and $\tau_{v}$ is the time of vibrational relaxation of excited molecules by buffer gas molecules. Condition (5.11) can be easily fulfilled if the buffer is monoatomic gas. In this case vibrational deactivation is possible only on account of a very slow process of $V-T$ relaxation. Second, when finding the value of $q$ with the use of relation (5.10) we should take into account the fact that the value of dissociation yield may be essentially acted upon by secondary collisional processes which, in their turn, may depend on pressure. So the data on the $q$ value obtained from measuring dissociation yield is sometimes only estimation by character.

Spectroscopic methods. The spectroscopic methods of determination of the fraction of molecules involved in the process of MP excitation are based on a difference in spectral characteristics of excited and unexcited molecules. The methods of spectroscopic diagnostics considered in Section 5.2 allow recording these variations and measuring 
directly and sometimes in combination with method of collisions the $q$ value and its dependence on the conditions of MP excitation.

The first measurements of the $q$ factor were taken for the $\mathrm{SF}_{6}$ molecule by the method of double IR-IR resonance. ${ }^{34}$ The linear absorption spectrum of $\mathrm{SF}_{6}$ was studied after the action of the IR pulse was over (Figure 5.10, curve 2), and the $q$ value was found from the formula

$$
q=1-S^{R} / S_{0}^{R}
$$

where $S_{0}^{R}$ is the integral intensity of the $R$-branch before the IR pulse is on, and $S^{R}$ is the integral intensity of the $R$-branch after the IR pulse is over. Such determination of the value of the $q$ factor holds for such molecules in which the anharmonic shift is larger than the width of the $R$-branch, for the $\mathrm{SF}_{6}$ molecule in particular (see Table 5.I).

Two-frequency dissociation of molecules is a modification of the method of double IR-IR resonance. The method of two-frequency dissociation can be also used to determine the $q$ value. It consists in the following. ${ }^{50}$ The molecules are excited at the resonant frequency $\Omega_{1}$ and then dissociate under nonresonant radiation at the $\Omega_{2}$ frequency. Let the nonresonant laser fluence be high enough so that the dissociation yield of the molecules originally excited by radiation at the $\Omega_{1}$ frequency is equal to unity. The absolute dissociation yield

TABLE 5.I

Some spectroscopic and energy parameters of $\mathrm{SF}_{6}$ molecule

\begin{tabular}{|c|c|}
\hline 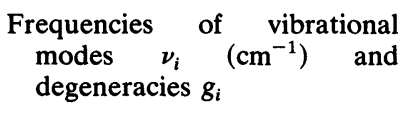 & $\begin{array}{l}\nu_{1}=774.53 ; \quad g_{1}=1 ; \quad \nu_{2}=643.35 ; \quad g_{2}=2 ; \quad \nu_{3}= \\
947.974,3307 ; g=3 ; \nu_{4}=615.03 ; g_{4}=3 ; \nu_{5}= \\
523.5 ; g_{5}=3 ; \nu_{6}=347 ; g_{6}=3\end{array}$ \\
\hline $\begin{array}{l}\text { Constants of intramode anhar- } \\
\text { monicity for } \nu_{3} \text { mode }\left(\mathrm{cm}^{-1}\right)\end{array}$ & $X_{33}=-1.83 ; G_{33}=1.34 ; T_{33}=-0.44$ \\
\hline $\begin{array}{l}\text { Constants of intermode anhar- } \\
\text { monicity for } \nu_{3} \text { mode } X_{3 j} \\
\left(\mathrm{~cm}^{-1}\right)\end{array}$ & $\begin{array}{l}X_{31}=-2.93-0.02 ; \quad X_{32}=-3.2-0.3 \\
\quad(-1.53-0.02) ; \quad X_{35}=(-0.5-1.0)^{\mathrm{a}} \\
-1.0-0.1\end{array}$ \\
\hline Rotational constant $B_{e}\left(\mathrm{~cm}^{-1}\right)$ & 0.09111 \\
\hline Constants of Coriolis splitting & $\begin{array}{r}\rho_{1}=(0)^{\mathrm{a}} ; \quad \rho_{2}=(0)^{\mathrm{a}} ; \quad \rho_{5}=0.63 \pm 0.004 ; \\
-0.213 \pm 0.01 ; \rho_{5}=(-0.5)^{\mathrm{a}} ; \rho_{6}=(-0.5)^{\mathrm{a}}\end{array}$ \\
\hline Dissociation energy & $32000 \pm 1000 \mathrm{~cm}^{-1}$ \\
\hline
\end{tabular}

${ }^{\text {a }}$ Estimation. 
in this case will produce the fraction of molecules $q$ excited by the field at the $\Omega_{1}$ frequency. As a matter of fact, it is necessary here that only the molecules excited by radiation at the $\Omega_{1}$ frequency should dissociate under the action of the $\Omega_{2}$ field. According to Ref. 50, this can be realized with a considerable displacement of $\Omega_{2}$ to the longwave side about the linear absorption band of the molecule.

In spontaneous RS probing of MP excited molecules the $q$ value can be determined by measuring both in the Stokes and anti-Stokes regions. In case of anti-Stokes IR-IR resonance to determine the $q$ value one should study the dependence of the anti-Stokes integral RS signal of any mode active in RS $\mathrm{S}^{\mathrm{AS}}$ on buffer gas pressure $p_{\text {buff }}$. According to Ref. 61, as $p_{\text {buff }}$ increases the value of $S^{\mathrm{AS}}$ grows and then reaches a constant. The increase in $S^{\mathrm{AS}}$ observed is provided by involving an increasing number of molecules into the process of excitation due to acceleration of rotational relaxation during an IR laser pulse. The fact that the dependence reaches its maximum value is conditioned by excitation of all the molecules to the quasicontinuum, and thus the maximum relative increase of $S^{\text {AS }}$ yields the value $q^{-1}$. It must be noted that this method of determination of $q$ is, in essence, fully identical to the method of collisions (expression (5.9)), since the integral intensity $S^{\mathrm{AS}}$ is proportional to the absorbed energy $\bar{\varepsilon}$ (see (5.5)).

In case of Stokes IR-IR resonance the $q$ value can be determined by observing two ensembles of molecules in the RS spectrum ${ }^{61}$ (like in case of double IR-IR resonance). It might be seen from Figure 4.2 in Ref. 2 for example, that the decrease in intensity of the nonshifted peak in the spectrum of Stokes RS signal of $\mathrm{SF}_{6}$ is conditioned by MP excitation of molecules. Therefore the $q$ value can be found from the formula

$$
q=1-S^{\mathrm{S}} / S_{0}^{\mathrm{S}}
$$

where $S_{0}^{\mathrm{S}}$ is the Stokes signal intensity before the IR pulse is on and $S$ is the nonshifted peak intensity of Stokes signal after the IR pulse is over. In studying of spontaneous IR fluorescence the $q$ value can be measured by a method completely similar to the one just described.

In case of IR-UV resonance the $q$ value can be determined with the use of the method of collisions. As shown in Ref. 52 with the $\mathrm{CF}_{3} \mathrm{I}$ molecule under study, the value of UV absorption induced by IR radiation from the long-wave side of the spectrum (Figure 5.11) 
depends on buffer gas pressure $\left(\mathrm{N}_{2}\right)$ in the following way: with increasing $p_{\text {buff }}$ the induced UV absorption grows at first and then reaches a constant. The maximum relative increase of induced UV absorption yields the value $q^{-1}$. Like in other methods of determination of $q$ based on the effect of collisions, it is assumed here that in the process of MP excitation two ensembles of molecules are formed and that the excitation level of the upper ensemble is independent of buffer gas pressure.

In conclusion, we may say the following about the methods of diagnostics of the process of passage of lower vibrational levels. These methods make it possible now to measure just the $q$ factor, that is an integrated characteristic of the process, but they cannot give any information yet on the distribution of the $q$ value over the initial quantum states of a molecule.

Besides, the question on the distribution in the lower, cold ensemble $F_{\text {cold }}\left(E_{\text {vibr }}\right)$ is open. Several experiments show, that some fraction of vibrationally excited molecules, for example $\mathrm{SF}_{6}{ }^{54,63,66}$ remain at lower discrete vibrational levels and do not pass into the quasicontinuum. For small molecules with high limit of quasicontinuum (for example $\left.\mathrm{SF}_{6}\right)$ this fraction may be significant. The information on $F_{\text {cold }}\left(E_{\mathrm{vibr}}\right)$ would make it possible to specify the mechanisms of passage of molecular lower vibrational states in MP excitation.

\subsubsection{Experimental results for different molecules}

Here we are going to present the results of measurements of the fraction of molecules involved into the process of MP excitation for some molecules. This data has been obtained mainly with the use of the above-described methods of collisions and spectroscopic methods. It should be pointed out that sufficiently complete information on the $q$ value and its dependence on the conditions of excitation has been obtained just for several molecules:

$S_{6}$ molecule. For this molecule the $q$ value has been measured by several different methods. The most comprehensive measurements have been carried out in exciting the $\mathrm{SF}_{6}$ molecule at room temperature in the region of the $Q$-branch of the $\nu_{3}$ band (the $P(16)$ line of the transition $00^{\circ} 1-10^{\circ} \mathrm{C}$ of $\mathrm{CO}_{2}$ laser). The results of these measurements are presented in Figure 5.19. The curve 1 has been produced by the 
method of double IR-IR resonance ${ }^{34}$. The value of $q$ increases slowly with increasing $\Phi$ in the range under measurement, and with $\Phi=$ $1.8 \mathrm{~J} / \mathrm{cm}^{2}$ it will be $q=0.7$. Such a slow dependence of $q$ on $\Phi$ follows also from the results of measurement of double IR-IR resonance spectra carried out in Ref. 39 with $\mathrm{SF}_{6}$ excited at another laser line- $P(20)$.

Figure 5.19 presents also the results of measurement of the $q$ value for $\mathrm{SF}_{6}$ by the method of double IR-RS resonance both in the Stokes and anti-Stokes regions. These data are in good agreement with the results of measurement by the method of double IR-IR resonance. In Figure 5.19 we can also see the value of $q$ obtained from comparing the values of absorbed energy in $\mathrm{SF}_{6}$ excited by pulses of different duration. ${ }^{9}$ The pulse with its duration $\tau_{p}=0.5 \mathrm{~ns}$ with $\Phi=1 \mathrm{~J} / \mathrm{cm}^{2}$ due to power broadening provides deliberately excitation of all the molecules, whereas with $\tau_{p}=100 \mathrm{~ns}$ and $\Phi=1 \mathrm{~J} / \mathrm{cm}^{2}$ not all the molecules but just their fraction $q$ is excited to the quasicontinuum. From this it follows that the value of the $q$ factor with $\tau_{p}=100 \mathrm{~ns}$ is equal to the ratio of the energy values absorbed from the $100 \mathrm{~ns}$ and $0.5 \mathrm{~ns}$ pulses. With $\Phi=1 \mathrm{~J} / \mathrm{cm}^{2}$, according to Ref. $9, q=0.5$ and it grows slowly with increasing laser fluence which agrees well with the results of other measurements. In Figure 5.19 the curve 2 is the dependence of $q$ on $\Phi$ obtained from relation (5.9) in measuring the value of $\bar{\varepsilon}$ as a function of pressure of $\mathrm{SF}_{6}$ gas by the method of opto-thermal calorimetry ${ }^{16}$ (Figure 5.17). This data corresponds to the collisionless conditions of excitation $\left(p=10^{-2}\right.$ Torr, $\left.\tau_{p}=100 \mathrm{~ns}\right)$.

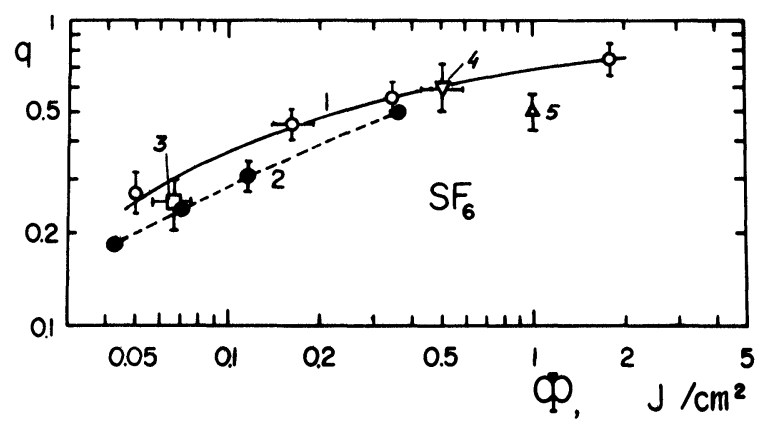

FIGURE 5.19 Dependence of $q$ value on laser energy fluence for $\mathrm{SF}_{6}$ molecule: (1) Ref. 34; (2) Ref. 16; (3) Ref. 59; (4) Ref. 60; (5) Ref. 9 (see text). 
TABLE 5.II

Certain results on double IR-IR-resonance for $\mathrm{SF}_{6}$ molecule

\begin{tabular}{|c|c|c|}
\hline Results & $\begin{array}{l}\text { Conditions of } \\
\text { MP excitation }\end{array}$ & Reference \\
\hline Rate of $V-T$ relaxation $p \tau_{V-T}=122 \mu \mathrm{S} \cdot$ Torr & $\begin{aligned} \Phi & =10^{-2} \mathrm{~J} / \mathrm{cm}^{2} \\
p & =0.2-4 \text { Torr }\end{aligned}$ & 35 \\
\hline Value of $p \tau_{V-T}$ is decreased with increasing of $\Phi$ & $\begin{array}{c}I<10^{6} \mathrm{~W} / \mathrm{cm}^{2} \\
P=1 \text { Torr }\end{array}$ & 36 \\
\hline Rate of rotational relaxation $p \tau_{\mathrm{rot}}=36 \mathrm{~ns} \cdot$ Torr & $\begin{array}{l}I=10^{-4} \mathrm{~J} / \mathrm{cm}^{2} \\
p=0.05-3 \text { Torr }\end{array}$ & 32 \\
\hline Direct measurement of power broadening & $\begin{array}{c}I \leqslant 10^{4} \mathrm{~W} / \mathrm{cm}^{2} \\
p=0.08 \text { Torr }\end{array}$ & $\begin{array}{l}32 \\
33\end{array}$ \\
\hline Observation of intramolecular relaxation & $\begin{array}{l}\Phi=0.02 \mathrm{~J} / \mathrm{cm}^{2} \\
p=0.1-1 \text { Torr }\end{array}$ & 37 \\
\hline Radiative depletion of many rotational states & $\begin{array}{c}\Phi>0.3 \mathrm{~J} / \mathrm{cm}^{2} \\
p=0.03-0.7 \text { Torr }\end{array}$ & 38 \\
\hline Red shift of MP induced absorption band & $\begin{array}{c}\Phi=0.1-2 \mathrm{~J} / \mathrm{cm}^{2} \\
p=0.05-0.8 \text { Torr }\end{array}$ & $\begin{array}{l}34,39 \\
40,41\end{array}$ \\
\hline $\begin{array}{l}\text { Existence of two ensembles at MP excitation. } \\
\text { Measurement of } q \text {-values }\end{array}$ & $\begin{array}{c}\Phi=0.16-1.8 \mathrm{~J} / \mathrm{cm}^{2} \\
P=0.18 \text { Torr }\end{array}$ & 34 \\
\hline $\begin{array}{l}\text { MP induced absorption at } 100 \mathrm{~cm}^{-1} \text { blue shift } \\
\text { with respect to } \nu_{3} \text { band }\end{array}$ & $\begin{array}{l}\Phi \leqslant 1 \mathrm{~J} / \mathrm{cm}^{2} \\
p=0.25 \text { Torr }\end{array}$ & 42 \\
\hline $\begin{array}{l}\text { Estimation of intermode interaction time } 1 \mathrm{~ns}< \\
\tau_{i}<30 \mathrm{ps}\end{array}$ & $\begin{array}{c}I<150 \mathrm{MW} / \mathrm{cm}^{2} \\
\tau_{p}=30 \mathrm{~ns}\end{array}$ & 43 \\
\hline
\end{tabular}

Therefore, the values of $q$ obtained are somewhat smaller than, for example, those obtained by other methods under excitation when collisions are not excluded completely.

$\mathrm{CF}_{3}$ I molecule Figure 5.20 presents some data on the dependence of the $q$ factor at MP excitation of the $\mathrm{CF}_{3} \mathrm{I}$ molecule on radiation fluence at the laser line corresponding to the $Q$-branch of the $\nu_{1}$ band of linear absorption. The curve 1 in Figure 5.20 obtained in Ref. 76 from comparing the results of the semiempirical model of MP excitation of $\mathrm{CF}_{3} \mathrm{I}$ (see Chapter VI) with the results of measurement of primary dissociation yield and absorbed energy in this molecule. In this case it is assumed in Ref. 76 that in MP excitation of $\mathrm{CF}_{3} \mathrm{I}$ like in case of $\mathrm{SF}_{6}$, there are two molecular ensembles formed: an ensemble of molecules remaining at lower vibrational levels and an ensemble of molecules excited to the vibrational quasicontinuum. The curve 2 
in Figure 5.20 is the dependence of $q$ on $\Phi$ obtained in Ref. 77 under definite simplifying assumptions of the type of dependence of primary dissociation yield on absorbed energy. Figure 5.20 also presents the values of $q$ at MP excitation of $\mathrm{CF}_{3} \mathrm{I}$ obtained by the methods of IR-UV and IR-RS resonances (see Section 5.2).

As seen from Figure 5.20, the value of the $q$ factor grows slowly with increasing laser fluence (like in case of $\mathrm{SF}_{6}$ ) and reaches almost unity with $\Phi=2-4 \mathrm{~J} / \mathrm{cm}^{2}$. The $q$ factor has maximum value in the region of maximum of linear absorption spectrum of $\mathrm{CF}_{3} \mathrm{I}$ (Figure 5.21) and is decreased by several times only at the far wings of the band.

$\mathrm{OsO}_{4}$ molecule The dependence of the $q$ value on IR laser fluence and frequency has been obtained for the $\mathrm{OsO}_{4}$ molecule in Ref. 50 by the method of two-frequency excitation. Figure 4.6 in Ref. 2 shows the dependence of the $q$ factor on laser frequency at the fixed value of IR pulse fluence $\Phi=1.2 \mathrm{~J} / \mathrm{cm}^{2}$ at the $\Omega_{1}$ frequency. In the region of absorption band maximum $q$ equals 0.6 and drops drastically as $\Omega_{1}$ shifts to the long-wave band edge. With increasing $\Phi$ there is an increase in $q$ which is much faster than in $\mathrm{SF}_{6}$ and $\mathrm{CF}_{3} \mathrm{I}$ (Figure 5.22).

Other molecules Table 5.III presents some known information on the value of the $q$ factor for a number of polyatomic molecules under conditions close to collisionless ones. It has been obtained mainly from the dependences of absorbed energy and dissociation yield on buffer gas pressure. It may be seen that for light few-atomic molecules the value of $q$ is much less than unity even in sufficiently intense IR fields.

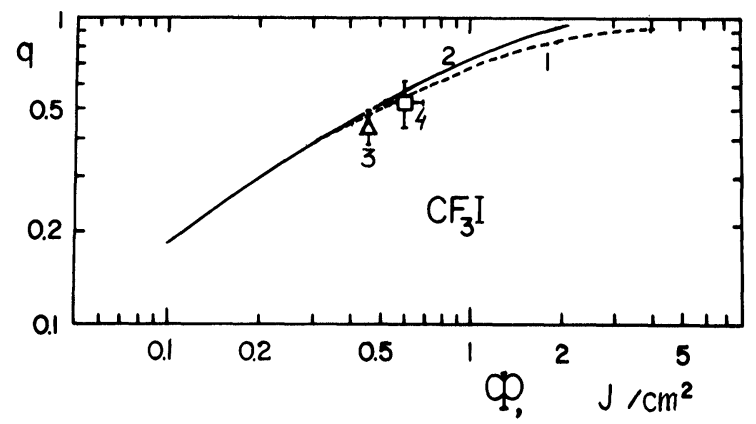

FIGURE 5.20 Dependence of $q$-value on radiation energy fluence for $\mathrm{CF}_{3} \mathrm{I}$-molecule: (1) Ref. 76; (2) Ref. 77; (3) Ref. 52; (4) Ref.. 60 (see text). 


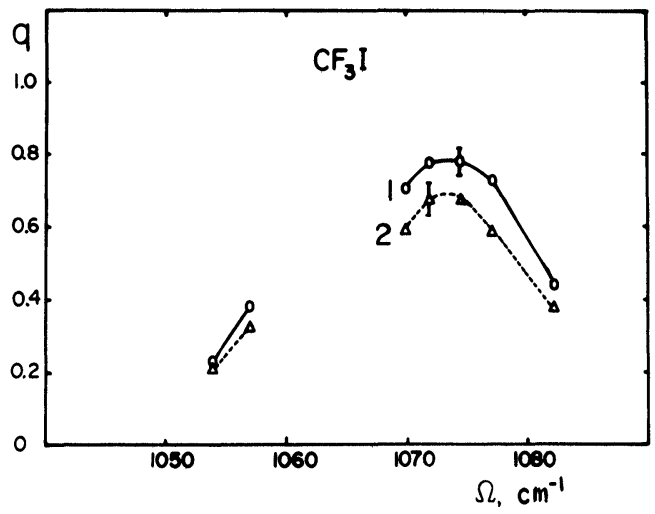

FIGURE 5.21 Frequency dependence of $q$-value for $\mathrm{CF}_{3} \mathrm{I}$ molecule: (1) Ref. 76; (2) Ref. 77 (see text).

At the same time, in case of more complex polyatomic molecules there is a considerable fraction of molecules involved in the process of MP excitation even in moderate-intensity fields.

It is possible to estimate rather roughly the value of the $q$ factor from comparing the value of absorbed energy at the dissociation threshold $\bar{\varepsilon}^{\text {thresh }}$ with the dissociation energy $D$. At the dissociation threshold the average energy of the molecules excited to the quasicontinuum $\bar{\varepsilon}_{q}^{\text {thresh }}$ should correspond to the dissociation energy by order of magnitude. It is the parameter $\chi$

$$
\chi=\bar{\varepsilon}^{\text {thresh }} / D
$$

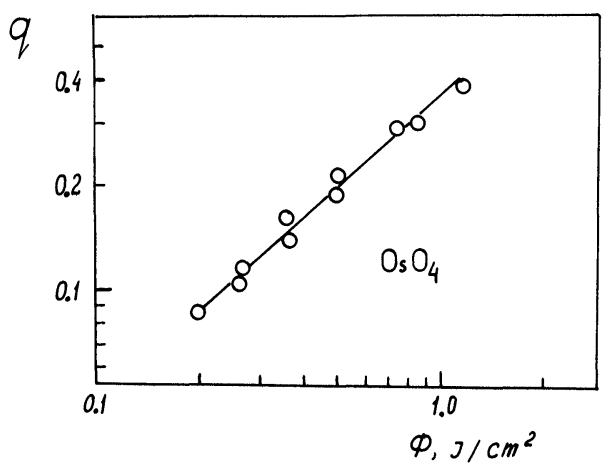

IGURE 5.22 Dependence of $q$ value on radiation energy fluence for $\mathrm{OsO}_{4}$ molecule: $\Omega=947.74 \mathrm{~cm}^{-1} ; p_{\mathrm{OsO}_{4}}=0.03$ Torr (from Ref. 50 ). 
TABLE 5.III

Values of $q$-factor for some molecules

\begin{tabular}{llcllc}
\hline & \multicolumn{2}{c}{ Conditions of excitation } & & \\
\cline { 2 - 3 } & $P($ Torr $)$ & $\Phi\left(\mathrm{J} / \mathrm{cm}^{2}\right)$ & & Reference \\
\hline $\mathrm{CDF}_{3}$ & 0.065 & 10 & 0.01 & 71 \\
$\mathrm{CF}_{2} \mathrm{HCl}$ & 0.02 & 2.4 & 0.1 & 73 \\
$\mathrm{C}_{2} \mathrm{H}_{3} \mathrm{~F}$ & 0.1 & 3 & 0.16 & 78 \\
$\mathrm{C}_{2} \mathrm{H}_{3} \mathrm{Cl}$ & 0.1 & 3 & 0.16 & 79 \\
$\mathrm{C}_{2} \mathrm{H}_{4} \mathrm{~F}_{2}$ & 0.05 & 40 & & 0.5 & 78 \\
$\mathrm{C}_{2} \mathrm{H}_{2} \mathrm{~F}_{2}$ & 0.05 & 40 & & 0.2 & 78 \\
$\mathrm{C}_{2} \mathrm{~F}_{3} \mathrm{Cl}$ & 0.1 & 4.4 & & 0.35 & 80 \\
& & 9.0 & 0.46 & \\
$\left(\mathrm{CF}_{3}\right)_{3} \mathrm{CBr}$ & 0.14 & 28 & 0.80 & \\
$\mathrm{Cyclobutan}_{2}$ & 0.1 & $0.1-80$ & $0.7-0.9$ & 81 \\
$\mathrm{C}_{2} \mathrm{~F}_{4} \mathrm{~S}_{2}$ & 0.1 & 1.0 & & 1.0 & 82 \\
\hline
\end{tabular}

that allows a very rough estimate of the $q$ value. Since at the dissociation threshold the average energy of the "hot" molecular ensemble $\bar{\varepsilon}_{q}^{\text {thresh }}$ is smaller than the dissociation energy, the $\chi$ parameter is the lower estimate for the value of the $q$ factor. Table 5.IV contains some estimates of $\chi$ parameter for a number of molecules. It follows from comparing these estimates with the results of direct measurement of the $q$ value that in all cases

$$
\chi \leqslant q
$$

TABLE 5.IV

Estimations of $\chi$-parameters for some molecules

\begin{tabular}{lcccl}
\hline \multicolumn{1}{c}{ Molecule } & $\bar{\varepsilon}^{\text {thresh }}\left(\mathrm{cm}^{-1}\right)$ & $D\left(\mathrm{~cm}^{-1}\right)$ & $\chi=\bar{\varepsilon}^{\text {thresh }} / D$ & \\
\hline $\mathrm{SF}_{6}$ & $15000^{84}$ & 32000 & 0.47 & \\
$\mathrm{SF}_{5} \mathrm{NF}_{2}$ & $2000^{19}$ & 17500 & 0.12 & \\
$\mathrm{~S}_{2} \mathrm{~F}_{10}$ & $3000^{8}$ & 20000 & 0.15 & \\
$\mathrm{OSO}_{4}$ & $1000^{85}$ & 27000 & 0.04 & At $\Omega=954 \mathrm{~cm}^{-1}$ \\
$\mathrm{CF}_{3} \mathrm{~J}$ & $4000^{77}$ & 18865 & 0.22 & \\
$\mathrm{CCl}_{4}$ & $28000^{86}$ & 35600 & 0.80 & For $\nu_{1}+\nu_{2}+\nu_{4}$ \\
$\mathrm{C}_{2} \mathrm{H}_{4}$ & $4000^{87}$ & 40000 & 0.10 & Value of $D$ is \\
$\mathrm{COC}_{2} \mathrm{~F}_{6}$ & $5000^{88}$ & 33000 & 0.14 & estimated in \\
& & & & Ref. 88 \\
\hline
\end{tabular}


In conclusion of this section it should be noted that the above results for the $q$ value enable quantitative comparison of the theoretical models of MP excitation in the quasicontinuum with the results of experiments. Besides, the experimental data on the $q$ value under really collisionless conditions of excitation can serve as one of the criteria for checking the theory of IR photoexcitation of lower vibrational molecular levels.

\subsection{Theoretical models of IR excitation of molecules at lower transitions}

The theory of excitation of lower vibrational molecular levels up to the vibrational quasicontinuum limit has been the subject of a lot of works in which very different models are being considered. There are some difficulties here. First, the available information on lower levels with $v>2$ is very scarce, and it is very difficult to get it. Second, in intense fields we should consider multistep and multiphoton transitions from a great number of vibrational-rotational states. Third, theoretical description must explain not only resonant characteristics of passage of lower levels but also the fraction of molecules coming into the quasicontinuum. The first stage of the process of MP excitation, that is passage of lower levels, is being studied and still far from completion. Here we are going to consider the available models, starting from the simplest ones, and to discuss in Section 5.4.5 what the theoretical models can give us to describe experiments.

\subsubsection{Model of one-dimensional anharmonic oscillator}

The oscillator model, as applied to excitation of molecular vibrations by laser radiation was considered as long ago as in Ref. 89. The oscillator is sure to be the simplest approximation for describing the energy spectrum of the given vibrational mode of a molecule. Unfortunately, even in the simplest case of one-dimensional motion the task cannot be solved precisely in the presence of anharmonicity. Nevertheless, due to numerous works where analytical methods and numerical calculations are applied we have got rather a complete picture at present.

The excitation dynamics of anharmonic oscillator in a monochromatic field is considered in Ref. 2 (Section 3.4). Usually under typical conditions of experiments on MP excitation of lower vibrational levels 
the value of power broadening is small as compared to the value of anharmonicity, i.e.,

$$
\gamma_{01} \ll 2 \omega^{i}\left|\chi_{i i}\right|
$$

(see the notations of Ref. 2). Therefore, in analysis of the model of anharmonic oscillator, as applied to the problem of passage of lower levels, it is sufficient to consider only multiphoton processes and neglect the effects of power broadening. To estimate the characteristic time of $n$-photon transitions we may use the results of Ref. 90 where the following parameters values are taken

$$
\mu_{01}=0.3 \mathrm{Deb}, \quad I=3 \cdot 10^{7} \mathrm{~W} / \mathrm{cm}^{2}, \quad 2 \omega^{i}\left|\chi_{i i}\right|=4 \mathrm{~cm}^{-1}
$$

For these parameters values typical of $\mathrm{MP}$ excitation of the $\mathrm{SF}_{6}$ molecule the value of population of $n$th level $\bar{\rho}_{n}=1$ can be obtained with $n=3$ in $1 \mathrm{ns,}$, with $n=4$ in 20 ns and with $n=5$ in 700 ns. Thus, the transition to states with $n \leqslant 4$ are expected to be effective enough for typical laser pulse durations $\tau \approx 100 \mathrm{~ns}$.

It follows from the dependence of the average population of the $n$th levels on exciting radiation frequency $\bar{\rho}_{n}(t)$, that at the frequency corresponding to the exact $n$-photon resonance $\bar{\rho}_{n}=\frac{1}{2}$. The frequency dependence $\bar{\rho}_{n}(t)$ must also develop resonances corresponding to multiphoton transitions to other levels, but at the frequencies of these resonances the value of $\bar{\rho}_{n}(t)$ is much less. This can be illustrated by the numerical calculation. ${ }^{90}$ The results of this calculation are given in Ref. 2 (Figure 3.7). Curve 1 corresponds to the case when $\gamma_{01} / 2 \omega^{i}\left|\chi_{i i}\right|=0.1$, i.e., when condition (5.16) is met. In the frequency dependence a distinct resonance structure manifests itself. As the field amplitude increases (curve 2 corresponds to the ratio $\gamma_{01} / 2 \omega^{i}\left|\chi_{i i}\right|=$ $0.4)$ the resonances are broadened materially.

When the field becomes strong enough so that the resonance halfwidth $\delta^{(n)}$ exceeds the anharmonic shift $2 \omega^{i}\left|\chi_{i i}\right|$ the multiphoton resonance frequency is no longer pronounced. In this case the quantum properties of the system are not so essential, and the classical approach to the solution of the task becomes justified. We do not consider here the classical limit since it does not correspond to the experimental conditions of molecular dissociation in fields with their intensity $I=$ $10^{7}-10^{9} \mathrm{~W} / \mathrm{cm}^{2}$. Here we should note only that full mathematical description of the theoretical methods in use can be found in Refs. 91 and 92 and specific estimates in Refs. 93 and 94. 


\subsubsection{Mechanisms of compensation of anharmonic shift at lower transitions}

Rotational compensation The possibility of compensation of anharmonicity becomes rather obvious, in principle, if the rotational motion of the molecule is taken into account. In the simplest approximation for spherical top molecules the energy levels of rotating harmonic oscillator can be presented in the form (2.33) (see Ref. 1). From the expressions for the frequencies of vibrational-rotational transition (2.48) and (2.49) allowed by the selection rules (2.39) we can have a unique possibility of resonant excitation of high-lying vibrationalrotational levels, if the laser frequency is tuned to any vibrationalrotational transition and the rotating anharmonic oscillator parameters satisfy the condition

$$
B_{v_{i}} \approx B=\omega^{i}\left|\chi_{i i}\right|
$$

In this case multistep resonances are possible which can excite the oscillator in sequence of $R$-transitions

$$
\begin{aligned}
&\left|v_{i}=0, J_{0}\right\rangle \stackrel{R\left(J_{0}\right)}{\longrightarrow}\left|1, J_{0}+1\right\rangle \\
& \stackrel{R\left(J_{0}+1\right)}{\longrightarrow}\left|2, J_{0}+2\right\rangle \cdots\left|n, J_{0}+n\right\rangle
\end{aligned}
$$

or $P$-transitions

$$
\begin{aligned}
&\left|v_{i}=0, J_{0}\right\rangle \stackrel{P\left(J_{0}\right)}{\longrightarrow}\left|1, J_{0}-1\right\rangle \\
& \stackrel{P\left(J_{0}-1\right)}{\longrightarrow}\left|2, J_{0}-2\right\rangle \cdots\left|n, J_{0}-n\right\rangle
\end{aligned}
$$

with exact compensation of anharmonic shift. Condition (5.18) for polyatomic molecules cannot be fulfilled even approximately, and usually

$$
B \ll \omega^{i}\left|\chi_{i i}\right|
$$

In the general case, when considering a sequence of $R$ - or $P$-transitions, we should simply use the effective anharmonicity $2\left(\omega^{i}\left|\chi_{i i}\right|-B\right)$ (see, for instance Refs. 93 and 94). In this case, with allowance made for anharmonicity variation, the estimates made for the case of onedimensional anharmonic oscillator without rotations remain valid. 
Yet, the situation changes if we consider a sequence of successive transitions in different branches. ${ }^{95}$ Indeed, when the radiation frequency is tuned to the $Q$-branch of the transition $v_{i}=1 \rightarrow v_{i}=2$, three successive vibrational-rotational transitions are possible (see Fig. 5.23)

$$
\begin{aligned}
\left|v_{i}=0, J=J_{r}\right\rangle \stackrel{P\left(J_{r}\right)}{\longrightarrow}\left|1, J_{r}-1\right\rangle \stackrel{Q\left(J_{r}-1\right)}{\longrightarrow}\left|2, J_{r}-1\right\rangle \\
\stackrel{R\left(J_{r}-1\right)}{\longrightarrow}\left|3, J_{r}\right\rangle
\end{aligned}
$$

Such a sequence of transitions, however, is possible only for molecules with a definite "resonant" value of rotational quantum number $\tau_{r}$ satisfying the condition

$$
2 B \tau_{r}=2 \omega^{i}\left|\chi_{i i}\right|
$$

It should be also noted that this process can take place if the initial state $\left|v_{i}=0, J_{0}=J_{r}\right\rangle$ is populated sufficiently. In the spectroscopic language this means that following condition ${ }^{95}$ must be fulfilled

$$
2 \omega^{i}\left|x_{i i}\right| \ll \delta \nu_{P}, \delta \nu_{R}
$$

where $\delta \nu_{P}, \delta \nu_{R}$ are the widths of the $P$ - and $R$-branches of the vibrational band under excitation. The possibility of triple stepwise resonance with rotational compensation of anharmonicity is rather

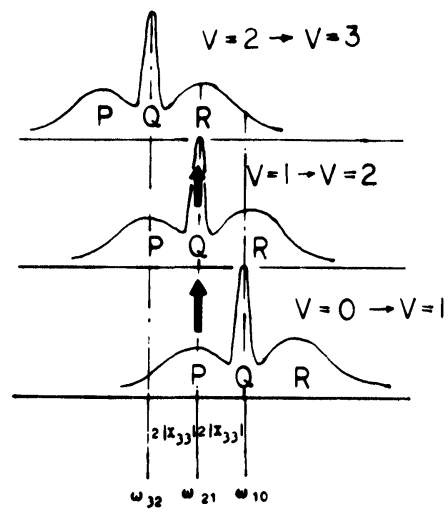

FIGURE 5.23 Triple $P Q R$ resonance on the three vibrational-rotational transitions. Field frequence coincides with $Q$-branch of $v=1 \rightarrow v=2$ transition. 
common but it is concerned only with excitation of the molecules from one rotational state at a rather strictly fixed frequency.

Rotational compensation of anharmonicity can also bring about an increase in efficiency of multiphoton resonances ${ }^{96}$ as compared to the case of an anharmonic oscillator without rotations. The point is that, with rotations allowed for, combinations of intermediate levels for multiphoton transitions with essentially smaller detunings from intermediate resonances become possible. The three-step $P Q R$ resonance considered above is in particular a degenerate case, when the detuning values at intermediate transitions are equal to zero. If at the same frequency $\Omega^{(3)}$ we consider the $P Q R$-transitions for molecules with $J \neq J_{r}$ it turns out (see Figure 5.24) that with $B \nu_{i}=B=$ const the $\Omega^{(3)}$ frequency is exactly equal to the three-photon resonant transition frequency. In Ref. 96 attention is drawn to the fact that the efficiency of such resonances as $P P R R$ and $P Q Q R$ is rather high. The Raby frequencies corresponding to such four-photon transitions for various values of $J$ can be found from the following formulas.

$$
\begin{aligned}
\gamma_{\text {eff }}^{(4)} & =\frac{\sqrt{6} \gamma_{01}^{4}}{8\left(3 \omega^{i}\left|\chi_{i i}\right|-2 B J\right)^{2}\left|2 \omega^{i}\right| \chi_{i i}|-B(2 J-1)|} \\
\gamma_{\text {eff }}^{(4)} & =\frac{\sqrt{6} \gamma_{01}^{4}}{8\left(3 \omega^{i}\left|\chi_{i i}\right|-2 B J\right)^{2}\left|2 \omega^{i}\right| \chi_{i i}|-B J|}
\end{aligned}
$$

According to the estimate made in Ref. 96 for the $\mathrm{SF}_{6}$ molecule, the Raby frequencies in a field with its intensity $I=2.5 \cdot 10^{7} \mathrm{~W} / \mathrm{cm}^{2}$ are $0.03 \mathrm{~cm}^{-1}$ or higher for most of the populated rotational sublevels of the ground vibrational state.

It follows from formulas $(2.48,2.49)$ in Ref. 1 that the frequency of any multiphoton transition in which the rotational quantum number is the same for the initial and final states, on condition that

$$
B_{n}=B=\text { const }
$$

does not depend on $J$. The frequency dependence of the absorption by $\mathrm{SF}_{6}$ calculated in Ref. 97 is concerned with assumption (5.27). The results of these calculations are presented in Figure 5.25. There are narrow resonances corresponding to multiphoton transitions of different multiplicity without variations in $J$. It should be noted, however, that equality (5.27) is not quite realistic for molecules. Indeed, from the linear absorption spectrum of the $\mathrm{SF}_{6}$ molecule (see Figure 2.4 


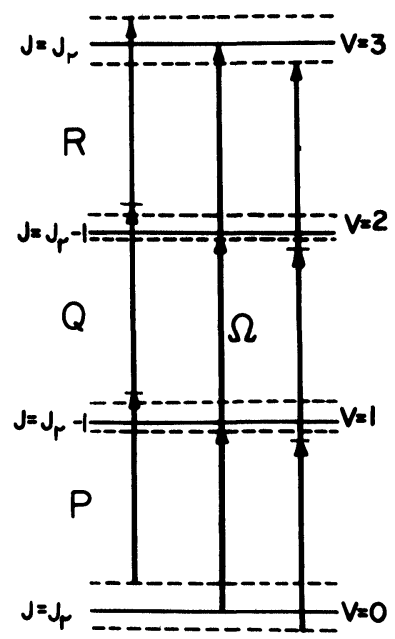

FIGURE 5.24 Three-photon $P Q R$-resonance. For resonance value $J_{r}$ the detunings at intermediate levels are equal to zero. For the others $J$-values the detunings are not equal to zero.

in Ref. 1) one can see that the $Q$-branch of the absorption spectrum has a final width $\geqslant 1 \mathrm{~cm}^{-1}$. The final width of the $Q$-branch is related to the variations in rotational constant (see formula (2.48), Ref. 1). The variations in rotational constant must cause, of course, the $Q$ branch of multiphoton transitions (i.e., multiphoton transitions with constant $J$ ) to be broadened, this broadening exceeding considerably the Raby frequency of multiphoton transitions in field with their intensities $I \approx 10^{7}-10^{9} \mathrm{~W} / \mathrm{cm}^{2}$. Therefore, we should conclude that in the model concerned, like in case of three-step PQR-resonance, at the given fixed frequency the field is able to excite effectively just a small fraction of lower rotational sublevels.

Anharmonic splitting There is another potential mechanism of compensation of anharmonicity at lower transitions for spherical top molecules (see, for example Refs. 98-100). It is well known that in these molecules in IR absorption there may be only triple-degenerate modes (the $\nu_{3}$ mode of $\mathrm{SF}_{6}$, for example). Inclusion of anharmonic terms in the Hamiltonian corresponding to the triple-degenerate mode brings about not only a usual shift of levels but also partial removal 


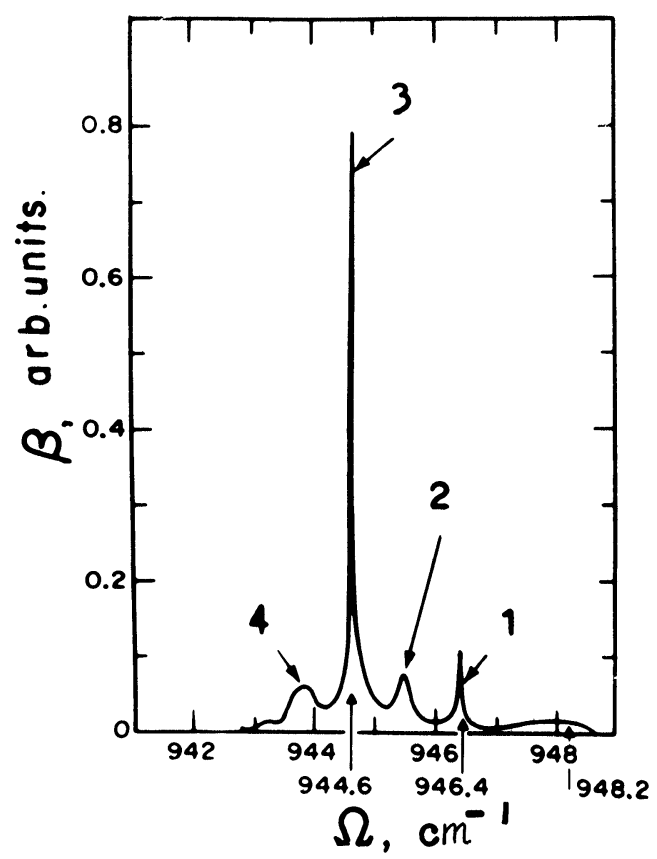

FIGURE 5.25 Frequency dependence of dissociation yield for $\mathrm{SF}_{6}$ molecule in the model of one dimensional anharmonic oscillator: (1) $Q$-branch of two-frequency transition $v=0 \rightarrow v=2$; (2) $R$-branch of three frequency transition $v=0 \rightarrow v=3$; (3) $Q$-branch of three-frequency transitions; (4) $P$-branch of three frequency transition. Raby frequency $\gamma_{01}=0.27 \mathrm{~cm}^{-1}$ (from Ref. 97).

of degeneration (see Section 2.2 in Ref. 1). It can be seen, for example, from the calculation of anharmonic splitting components for the vibrational levels of the $\mathrm{SF}_{6}$ molecule (see Figure 2.2 in Ref. 1) that the splitting value can be compared with the anharmonic shift value. Therefore, the effect of anharmonic shift can promote compensation of anharmonicity. It is also expected that new multiphoton resonances can appear due to anharmonic splitting. To understand to what extent the effect of anharmonic splitting complicates the spectral structure of the transitions between vibrationally excited states we should consider the selection rules for electric dipole transitions. With spherical symmetry preserved (i.e., with the anharmonic constant $T_{33}=0$ ), the following selection rules take place

$$
\Delta n= \pm 1, \quad \Delta l= \pm 1
$$


i.e., two upward transitions are possible for each component with $l \neq 0$ and one transition for components with $l=0$. With $T_{33} \neq 0$ the possibility of transition from the octahedral substate $p^{\prime}$ of the vibrational state $\left[n \omega_{3}\right]$ to the octahedral substate $p^{\prime \prime}$ of the vibrational state $\left[(n+1) \omega_{3}\right]$ is determined by the standard rule: the direct product $p^{\prime} \times p^{\prime \prime}$ must contain the representation $F_{1 u}$ of the $O_{h}$ group. This rule leads to allowed transitions (2.58) (see Ref. 1, Section 2.2.3). It is of interest to compare the frequency dependences of oscillator excitation without and with anharmonic splitting. Such a type of calculation is illustrated in Figure 5.26. One can observe an essential difference in the spectral structure that is related to a much richer structure of multiphoton resonances for the case of anharmonic splitting.

This structure in the spectrum, as well as in the above case of oscillator without allowance of anharmonic splitting, may be camouflaged by the rotational motion of molecules. Rotational motion causes (see Ref. 10) each component of anharmonic splitting to get a rotational structure. For the $A$ - and $E$-sublevels the contribution of rotations is similar to the rotational energy component in expression (2.33) in Ref. 1. For the $F$-sublevels Coriolis interaction takes place

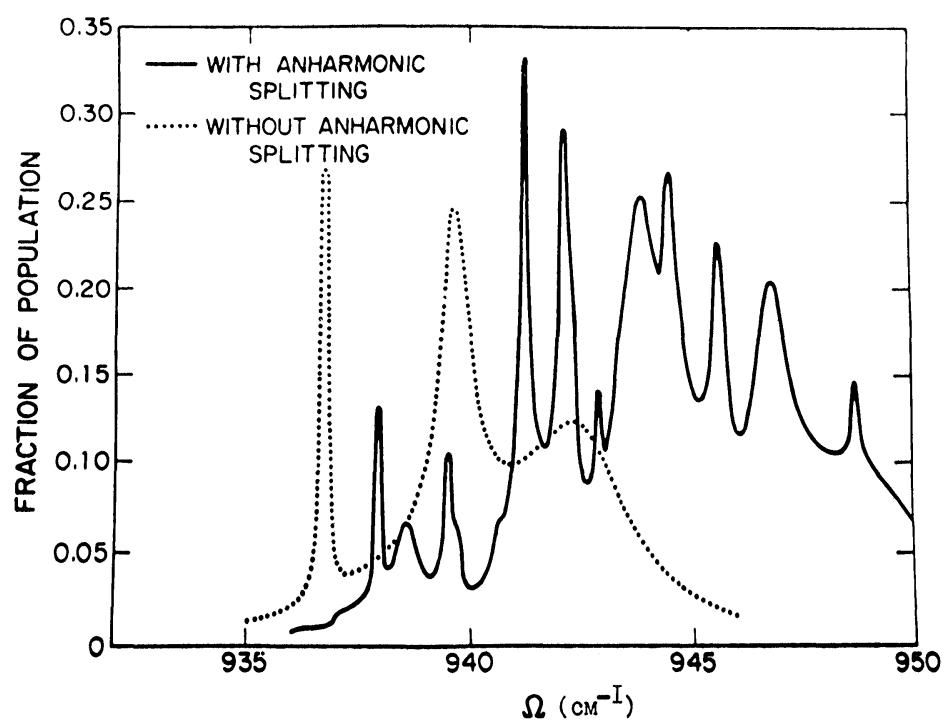

FIGURE 5.26 Frequency dependence of populations the $v=4 ; 5$ levels for $\mathrm{SF}_{6}$ molecule. Raby frequency $\gamma_{01}=3 \mathrm{~cm}^{-1}$. 
which causes the level with the given value of total angular momentum $J$ to split into three components characterized by the values of quantum number $R=J+1, J, J-1$. The expression for vibrational level energies can be written in an analytical form for the case of spherically symmetric anharmonicity (i.e., with $T_{33}=0$ ):

$$
\begin{aligned}
E(n, l, J, R)= & n \omega_{3}^{(0)}+n(n-1) X_{33}+\left[l(l+1)-2 n G_{33}+B J(J+1)\right. \\
& +B \xi[R(R+1)-J(J+1)-l(l+1)+2 n]
\end{aligned}
$$

The notations used here are similar to those in Ref. 1 . The additional selection rules for electric dipole transitions relative to rotational quantum numbers are following:

$$
\Delta J=0, \pm 1 ; \quad \Delta R=0 .
$$

The dipole moments of allowed transitions calculated in Ref. 102 have been used to estimate the frequency dependence of excitation of the $\mathrm{SF}_{6}$ molecule in Refs. 103-105. Figure 5.27 compares the results of calculations in Ref. 103 for the model taking no account of anharmonic splitting and the model of spherically symmetric splitting. The authors state that at the gas temperature $T=300 \mathrm{~K}$ the results are not responsive to anharmonic splitting. However, the experiment in Ref. 24 on measuring the frequency dependence of absorption in $\mathrm{SF}_{6}$ with a tunable $\mathrm{CO}_{2}$ laser at a low temperature, $T=170 \mathrm{~K}$, has revealed a structure in the multiphoton absorption spectrum related by the authors to anharmonic splitting. So further studies are necessary here.

Symmetrical top The possibility of compensation of anharmonicity considered here which is connected with anharmonic splitting relates to the triple-degenerate modes of spherical top molecules. There has been considered one more potential mechanism of compensation anharmonicity relating to the perpendicular absorption bands of symmetrical top molecules. ${ }^{4}$ A symmetrical top has two rotational constants $B_{v_{i}}$ and $A_{v_{i}}$, and its energy in a simplest approximation are given by expression (2.10) in Ref. 1 , where $K$ is the projection of angular momentum onto the molecular axis $(-J \leqslant K \leqslant J)$. The selection rules for electric dipole transitions for perpendicular bands allow variations not only in $J$ but also in $K: \Delta J=0, \pm 1 ; \Delta K= \pm 1$. Therefore, there are some more possibilities for variations in rotational energy during vibrational transitions. From (2.51) in Ref. 1 it follows, in 


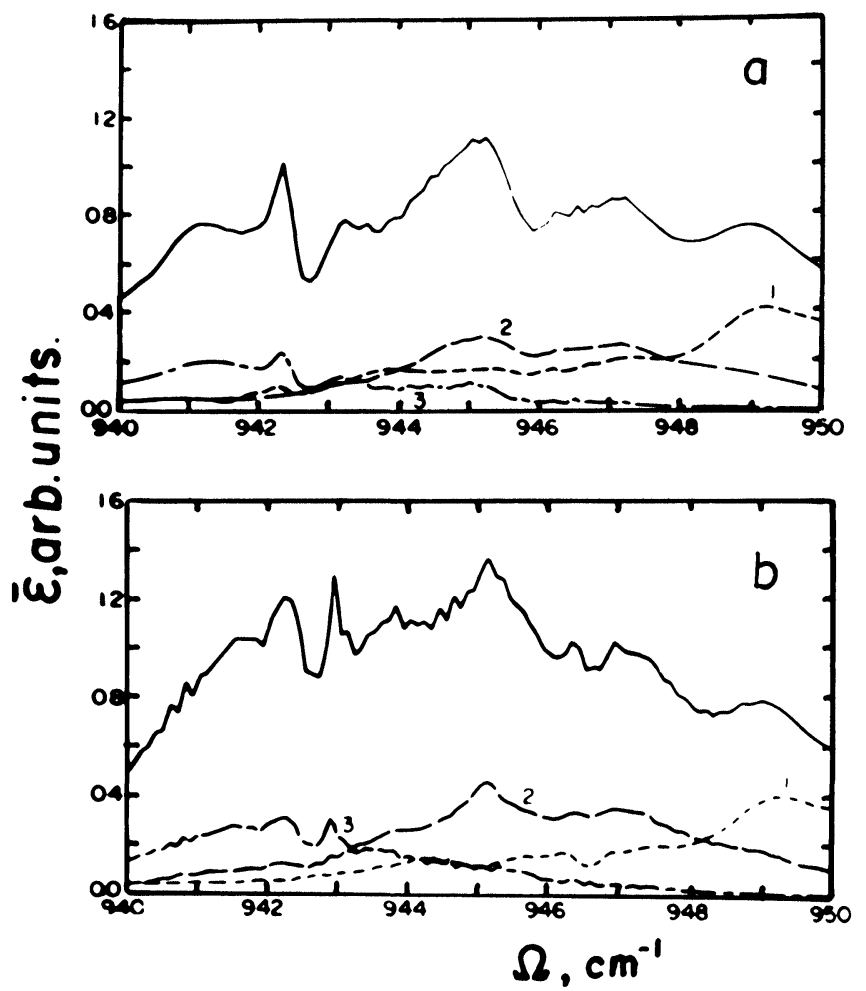

FIGURE 5.27 Frequency dependence of absorbed energy in $\mathrm{SF}_{6}$, calculated for really excited vibrational-rotational distribution at $T=300 \mathrm{~K}$ : (a) without anharmonic splitting of $\nu_{3}$ mode; (b) in the model of spherical symmetric splitting (from Ref. 103).

particular, that when the condition

$$
A-B=\left|\chi_{i i}\right| \omega^{i}
$$

is fulfilled, the following multiple resonance on the field frequency with a sequence of vibrational transitions can occur (see Figure 5.28)

$$
\begin{aligned}
\left|v_{i}=0, J_{0}, K_{0}\right\rangle & \stackrel{P Q_{J_{0}}}{\longrightarrow}\left|1, J_{0}, K_{0}-1\right\rangle \stackrel{P Q_{J^{-1}}}{\longrightarrow}\left|2, J_{0}, K_{0},-2\right\rangle \\
& \longrightarrow \cdots\left|K_{0}, J_{0}, 0\right\rangle \stackrel{R Q_{0}}{\longrightarrow}\left|K_{0}+1, J_{0},-1\right\rangle \\
& \longrightarrow \cdots\left|2 F_{0}, J_{0},-K_{0}\right\rangle
\end{aligned}
$$




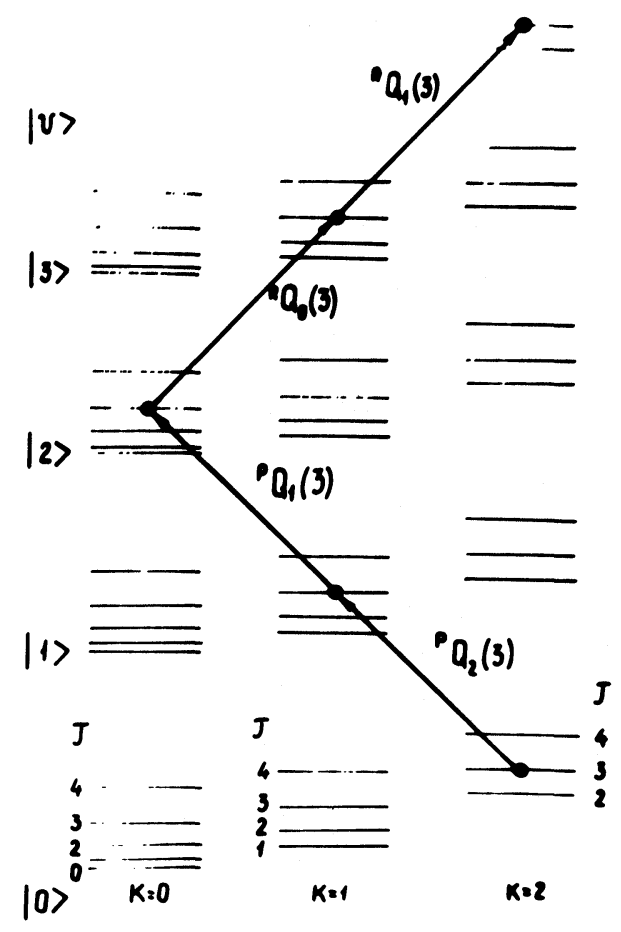

FIGURE 5.28 Illustration for the possibility of rotational compensation of anharmonicity on vibrational-rotational transitions of perpendicular branches for symmetrical top molecules, Eq. (5.31) being valid (from Ref. 4).

For such a $2 K_{0}$-fold vibrational-rotational resonance the field frequency must be tuned to the frequency of the $P_{Q_{K_{0}}}$-transition. It is the value of quantum number $K$ in the initial state that determines the number of possible successive resonances. Condition (5.31) for symmetrical top molecules can be fulfilled with much a higher probability than the similar condition (5.23) for spherical top molecules because the rotational constant $A$ may be much larger than $B$ and can be compared with the anharmonicity constant. Such a multiple resonance may arise at every coincidence of the laser radiation frequency with the center of the $P_{Q_{K_{0}}}$-branch. So the multiphoton absorption spectrum in case of such a mechanism of compensation of anharmonicity must have a periodic structure. Such a structure of multiphoton absorption spectrum has been disclosed for the perpen- 
dicular $\nu_{7}$ band of the $\mathrm{C}_{2} \mathrm{H}_{4}$ molecule ${ }^{4}$ which suggests a possibility of the process of excitation (5.32).

It must be noted, however, that for the parallel bands of symmetrical top molecules (such bands are excited, for example, in experiments with $\mathrm{CF}_{3} \mathrm{I}^{106}$ and $\mathrm{CF}_{3} \mathrm{Br}^{107}$ ) the following selection rule is fulfilled: $\Delta K=0$. Therefore the transition frequencies are described by formulas from $(2.48,2.49)$ in Ref. 1 , and there is just one possibility of compensation anharmonicity, that is a successive $P Q R$-resonance. The parallel bands of symmetrical top molecules have the simplest structure. So we can expect that henceforth the comparison of the results of experiments on excitation of these bands with the results of experiments in more complex cases will enable choosing a mechanism of compensation of anharmonicity for a particular molecule.

\subsubsection{Model of multiphoton transition to the quasicontinuum}

It is already pointed out that the key moment in describing the process of MP excitation is the representation of vibrational quasicontinuum, that is an energy region where stepwise resonant transitions can take place up to the dissociation limit. The presence of a quasicontinuous spectrum of transitions for rather high-lying vibrational levels (in more detail this question is considered in Ref. 1 (Section 2.3)) makes apparently possible a multiphoton resonant transition from the ground state directly into the quasicontinuum. It is interesting to note that in one of the very first works ${ }^{108}$ concerned with the theory of the process of MP excitation consideration was given to multiphoton transitions directly to the quasicontinuum lying above the dissociation limit. This mechanism of molecular excitation (see Ref. 2, Section 3.5.5) can be understood quite easily in the language of quantum-mechanical leakage or tunneling. ${ }^{98,109-112}$

Let us consider the $|a\rangle$ level the energy of which differs from the conventional lower limit of vibrational quasicontinuum by less than the energy of one laser IR radiation quantum. In the general case, when this level is not coupled by resonances with any populated vibrational-rotational molecular level and when the laser intensity is not sufficient to excite highly this level due to power broadening, its population $\rho_{a a} \ll 1$. This population can be estimated within the frameworks of the perturbation theory with due regard for the transitions nearest to resonant ones. But, despite the equality $\rho_{a a} \ll 1$, if 
during a laser pulse many elementary acts of irreversible decay of the level to the quasicontinuum take place, the effect of molecular excitation may be rather appreciable. The rate of "leakage" from lower states to the quasicontinuum is given by the obvious relation

$$
W=\rho_{a a} \sigma_{a} P
$$

where $\sigma_{a}$ is the cross-section of transitions from the $|a\rangle$ level to the quasicontinuum, $P$ is the laser radiation intensity in photon $/ \mathrm{cm}^{2} \cdot \mathrm{s}$. The probability of leakage during a pulse is ${ }^{11}$

$$
\xi=1-\exp \left(-W \tau_{p}\right)=1-\exp \left(-\rho_{a a} \sigma_{a} P \tau_{p}\right)
$$

This mechanism of molecular excitation to the quasicontinuum in some papers ${ }^{113-116}$ was considered in a different way, that is, in terms of decay of the anharmonic mode under excitation to other vibrational degrees of freedom of the molecule.

The essence of the effect, however, is reflected obviously most precisely by considering in terms of direct multiphoton transitions to the quasicontinuum. An important feature of cross-over multiphoton transitions to the quasicontinuous spectrum is that it is possible to excite all molecules irrespective of their initial state. The transition probabilities for different initial states are dissimilar, of course, and as this always takes place in multiphoton processes (see, for example, Refs. 117 and 118), depend on the value of detuning $\Delta_{K}$ relative to intermediate resonances (see Figure 3.14 in Ref. 2). The probability of such a process is determined by formula (3.134) in Ref. 2. Generally speaking, this formula is rigorous for multiphoton transitions to the quasicontinuum. But, if the probability of transition to the continuum is much larger than the distance between the levels in it, formula (3.134) holds true for the case of transition to the quasicontinuum, too.

Let us consider for estimation a four-photon transition to the quasicontinuum through the most advantageous channel PQQR (see Section 5.5.2). Assume that the dipole moment of transition $\mu=$ $0.3 \mathrm{Deb}$ and anharmonicity constant $\omega^{i}\left|x_{i i}\right|=2.5 \mathrm{~cm}^{-1}$. Let, also, the pulse duration $\tau_{p}$ be $100 \mathrm{~ns}$, and the cross-section of transition to the quasicontinuum $\sigma=10^{-18} \mathrm{~cm}^{2}$ (see Chapter 6). Then with the use of formula (3.134) we can have the characteristic intensity of laser radiation $I \simeq 10^{8} \mathrm{~W} / \mathrm{cm}^{2}$ at which all the initial states are excited to the quasicontinuum with a probability close to unity $\left(W \tau_{p} \sim 1\right)$. 
The estimate obtained is quite consistent with experiments at such intensities when some molecules (see Section 5.4) can be excited to the quasicontinuum with a probability close to unity. But, according to formula (3.134) in Ref. 2, as the intensity is reduced just by several times the probability of excitation to the quasicontinuum in a laser pulse becomes much less than unity. This is quite natural since the model of multiphoton transitions to the quasicontinuum gives a fast stepwise dependence (in our case $I^{4}$ ) of absorption on laser radiation intensity which can be illustrated by the results of numerical computations. ${ }^{97112,119}$ We should also point to another characteristic feature of the process of direct multiphoton excitation to the quasicontinuum. The efficiency of this process, according to Refs. 110,112 and 120 is determined mainly by the intensity of the laser pulse rather than by its energy.

\subsubsection{Model of weak transitions}

In some works the effect of MP excitation has been explained with the use of transitions forbidden by the selection rules discussed in Section 5.5.2 but which can be essential in a sufficiently strong laser field.

Transitions with $|\Delta J|>1$ In Ref. 121 consideration was given to vibrational-rotational transitions with $|\Delta J|>1$. It is rather easy to understand why we should allow for such transitions in intense fields. Transitions with $|\Delta J|>1$ may be treated, in essence, as multiphoton transitions (see Figure 5.29) the intermediate stages of which are

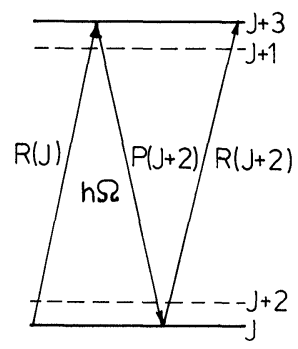

FIGURE 5.29 Three-photon transitions with $\Delta J=-3$ from the ground vibrational state to the first excited vibrational state. The levels $\left|v=1, J=J_{0}-1\right\rangle$ and $\mid v=0$, $J=J_{0}-2$ ) play the role of intermediate state. 
allowed but detuned from exact resonances. The effective Raby frequencies of these transitions can be easily calculated with formulas similar to those given in Section 5.4.2 which include the dipole moments of all intermediate transitions as well as the detunings from intermediate resonances. Let us consider, for example, the transition with $\Delta J=-3$. As it follows from formula (2.33) in Ref. 1, the frequency of this transition is

$$
\omega_{10}^{J_{0}-3}=\omega^{i}-6 B\left(J_{0}-1\right)
$$

where it is assumed that $B_{0}=B_{1}=B$. The transition with $\Delta J=-3$ corresponds to a three-photon transition in which the levels $\mid v_{i}=1$, $\left.J=J_{0}-1\right\rangle$ and $\left|v_{i}=0, J=J_{0}-2\right\rangle$ act as intermediate states. The effective Raby frequency of this transition is expressed as

$$
\gamma_{\mathrm{eff}}^{(3)}=\frac{\gamma_{01}^{3}}{16 B^{2}(2 J-1)(2 J-3)}
$$

where, for simplicity, we neglect the weak dependence of the vibrational-rotational transition dipole moment on the rotational quantum number $J$. If we take the values of transition dipole moment $\mu=$ 0.3 Debye, laser radiation intensity $I=10^{7} \mathrm{~W} / \mathrm{cm}^{2}$ and rotational constant $B=0.1 \mathrm{~cm}^{-1}$ we shall have the Raby frequency

$$
\gamma_{\text {eff }}^{(3)} \geqslant 0.5 \cdot 10^{-4} \mathrm{~cm}^{-1} \text { (or } \gamma_{\text {eff }} \cdot 2 \pi c \geqslant 10^{7} \mathrm{~s}^{-1} \text { ) for } J \leqslant 45 \text {. }
$$

Thus, transitions with $|\Delta J|>1$ must be apparently taken into account with the rest of multiphoton transitions, particularly for small values of the rotational quantum number $J$.

Transitions with $\Delta R \neq 0$ Quite a different approach must be applied in considering the removal of prohibition $\Delta R=0$ on the quantum number $R$ which takes on the values $J+1, J, J-1$ for different components of Coriolis splitting of the $F$-sublevel of spherical top molecules. It is essential here to clear up to what extent the selection rule $\Delta R=0$ is rigorous. Transitions with $\Delta R \neq 0$ have observed in the linear absorption spectra of the $\mathrm{CH}_{4}$ molecule. ${ }^{122,123}$ Therefore in Refs. 124, 125 and 116 an assumption has been made on the possible role of such transitions in the process of MP excitation of spherical top molecules. In Ref. 127 the mechanism of removal of prohibition $\Delta R=0$ has been revealed. 
It has been shown that the selection rule $\Delta R=0$ can be disturbed in the highest orders of vibrational-rotational interaction. But, as shown in Ref. 127 , it is impossible at present to carry out exact calculations of the dipole moments of transitions with $\Delta R \neq 0$ for lack of information on many molecular parameters. Nevertheless, according to the approximated estimations in this work, the probabilities of transitions with $\Delta R \neq 0$ for heavy molecules $\left(\mathrm{SF}_{6}, \mathrm{UF}_{6}\right)$ make $\left(10^{-8}\right.$ $\left.10^{-10}\right) J^{4}$ of the probability of allowed transitions with $\Delta R=0$. For the $\mathrm{SF}_{6}$ molecule the upper estimate means that the dipole moments of transitions with $\Delta R \neq 0$ at $J \sim 50$ are expected to be $\mu \simeq$ $0.7 \cdot 10^{-2}$ Deb. Even if the laser radiation intensity $I \simeq 10^{5} \mathrm{~W} / \mathrm{cm}^{2}$ such a value results in the Raby frequency value $\gamma \simeq 10^{-4} \mathrm{~cm}^{-1}$ (or $\gamma 2 \pi c \simeq 2 \cdot 10^{7} \mathrm{~s}^{-1}$ ), i.e., weak transitions with $\Delta R \neq 0$ can be probably saturated even in moderate-intensity fields.

Transitions with $\Delta n \neq 0$ There is one more non-trivial possibility of weak transitions for spherical top molecules. To understand what this possibility is connected with we should consider the fine rotational structure of a sublevel with the given value of $J$. In a spherical symmetry approximation, beside the usual degeneracy with respect to the projection of the angular momentum $M$ onto the given spatial axis, there is also degeneracy with respect to the projection $K$ in the coordinate system rigidly connected with the molecule. Anisotropic vibrationalrotational interaction removes the degeneracy with respect to $K$, which gives birth, in particular, to a structure observed in linear absorption spectra measured with a high resolution (see Figure 2.6 in Ref. 1). As it may be seen from this figure, in the absorption spectrum of $\mathrm{SF}_{6}$ the bands corresponding to different values of $J$ are overlapped rather materially. Each line in the absorption spectrum is classified with the irreducible representation of an octahedral group. This splitting was theoretically studied as far back as in classical Ref. 128 where it was shown that of each octahedral component of the lower vibrationalrotational state in the given branch $(P-, Q-$ or $R$-branches) there was only one transition to a strictly definite octahedral component of the upper vibrational-rotational state that was allowed. In the notations of Ref. 128 the octahedral components of the $C$ symmetry are numerated by the $n$ index and the selection rules $C^{\prime}=C^{\prime \prime}, \Delta n=0$ are valid. But, according to this work, the second rule is not rigorous. Therefore in Ref. 126 it has been assumed that it is transitions with $\Delta n \neq 0$ that 
are essential for the process of MP excitation. The theoretical model of MP excitation including transitions with $\Delta n \neq 0$ has been considered in Ref. 129 too. The physical results obtained from considering transitions with $\Delta n \neq 0$ are quite evident. Quite a large number of transitions $(\sim J)$ becomes possible for the given component of octahedral splitting. This, of course, may cause a considerable fraction of molecules to be involved in the process of resonant interaction with the field at a fixed frequency. Figure 5.30 shows the frequency dependence, estimated in Ref. 126, of the fraction of molecules excited at the transition $v=0 \rightarrow v=1$ with allowance made for weak transitions with $\Delta n \neq 0$ (as well as with $\Delta R \neq 0$ ). The figure also shows the frequency dependence of $q$ for the case when weak transitions are absent. This comparison shows that, with weak transitions allowed for, the value of $q$ may increase by more than an order. For further progress it is apparently to clear up to what extent the selection rule $\Delta n=0$ is rigorous. Theoretical estimations in this direction require that many unknown molecular parameters should be known. So it is apparent that detailed spectroscopic research into transition cross-sections and level positions is essential for the development of a model of weak transitions.

\subsubsection{Experimental testing of theoretical models}

The experimental results of studies into the process of MP excitation have at least three aspects which are most essential for testing the

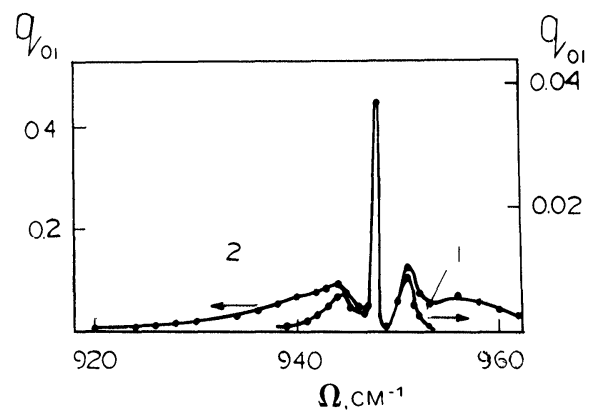

FIGURE 5.30 Frequency dependence of $q$-factor at the excitation of $\mathrm{SF}_{6}$ molecules from the ground to the first excited vibrational state: (1) calculation with $\Delta R=0$, $\Delta n=0$ selection rules taking into account; (2) Calculation with assume the breaking $\Delta R=0, \Delta n=0$ selection rules (from Ref. 126). 
models developed for IR excitation of molecules at lower transitions. The first of these aspects is the frequency dependence of absorbed energy which actually determines the selectivity of MP excitation and are very significant for the practical applications of the effect. To conclude that the theory operates here rather well it is apparently quite sufficient to compare the experimental frequency dependence of absorption in $\mathrm{SF}_{6}$ (Figure 4.4 in Ref. 2) with its associated theoretical dependences (Figure 5.28) calculated for the real gas temperature. The second and third aspects, the observed values of energy absorbed from a laser pulse and the observed values of the fraction of molecules $q$ excited to the quasicontinuum, are closely interrelated. Indeed, the possibilities of excitation of a small fraction of molecules associated with different mechanisms of compensation of anharmonicity (see Section 5.4.2) are rather evident, and these molecules are able to absorb several quanta and then go on with their excitation in the quasicontinuum. The possibility of excitation of a considerable fraction of molecules, however, is non-trivial (according to Section 5.3, the value of $q$ for $\mathrm{SF}_{6}, \mathrm{OsO}_{4}, \mathrm{CF}_{3} \mathrm{I}$ molecules at rather moderate radiation intensities $I \simeq 10^{6} \mathrm{~W} / \mathrm{cm}^{2}$ ranges from $0.1-0.5$ ). The mechanism of compensation of anharmonicity considered in Section 5.4.2 are not able apparently to provide so high values of the $q$ factor. In Refs. 103-105, for example, in which the process of MP excitation in the $\mathrm{SF}_{6}$ molecule is calculated in detail, there is no quantitative correlation with the experimental dependences $\bar{\varepsilon}(\phi)$ and $q(\phi)$. This fact enables us to assume that the authors have not managed to solve the problem but, nevertheless, the results of their calculations are of great interest.

Since the models of compensation of anharmonicity considered in Section 5.4.2 seem to be insufficient to describe experiments we had to use other techniques considered in Sections 5.4.3 and 5.4.4. The mechanism of direct multiphoton transitions to the quasicontinuum discussed in Section 5.4.3 reflects properly an important effect of the process of MP excitation, that is the possibility of excitation of any initial molecular state when the power broadening is still relatively small. But, as the estimates given above show, this mechanism results in a fast dependence of $q$ on laser radiation intensity which differs completely from those observed in experiments. The same refers to the corresponding dependence of absorbed energy.

The dependences $\bar{\varepsilon}(\phi)$ and $\beta(\phi)$ formally look as if stepwise resonant transitions would be possible for many initial molecular states. 
It is because of this that weak transitions have been considered (see Section 5.4.4). We can state that the most cardinal assumption here is distortion of the selection rule $\Delta n=0$ that gives rise to excitation channels the number of which exceeds at least by an order the number of channels considered above. The model of weak transitions with $\Delta R \neq 0, \Delta n \neq 0$ has not been developed properly yet. Besides, it should be noted that this model is rather specific since it deals only with excitation of triple-degenerate modes of spherical top molecules. But, as far as other molecules and particularly asymmetric tops are concerned, most of the scientists believe that the difficulties of the theory in describing the excitation of lower transitions are caused by lack of spectroscopic information. Further studies will show to what extent this opinion is true.

\section{References}

1. V. N. Bagratashvili, V. S. Letokhov, A. A. Makarov and E. A. Ryabov, Laser Chemistry (in press).

2. V. N. Bagratashvili, V. S. Letokhov, A. A. Makarov and E. A. Ryabov, Laser Chemistry (in press).

3. V. P. Zharov and V. S. Letokhov, Laser Opto-Acoustical Spectroscopy, in: Springer Series in Optical Sciences (Springer, Berlin, Heidelberg, New York, in press).

4. V. N. Bagratashvili, I. N. Knyazev, V. S. Letokhov and V. V. Lobko, Optics Commun. 18, 525 (1976).

5. V. N. Bagratashvili, V. P. Zharov and V. V. Lobko, Kvantovaya Electron. (Russian) 5, 637 (1978).

6. K. Hartung and R. Yurgeit, Kvantovaya Electron. (Russian) 5, 1825 (1978).

7. R. V. Ambartzumian, L. M. Dorozhkin, G. N. Makarov and B. A. Chajanov, Appl. Phys. 22, 409 (1980).

8. J. L. Lyman and K. M. Leary, J. Chem. Phys. 69, 1858 (1978).

9. J. G. Black, P. Kolodner, M. J. Shultz, E. Yablonovitch and N. Bloembergen. Phys. Rev. A 19, 404 (1979).

10. A. Yariv, Quantum Electronics (John Wiley and Sons, New York, London, Sydney, 1967).

11. Yu. R. Kolomijsky, V. S. Letokhov and O. A. Tumanov, Kvantovaya Electron (Russian) 3, 1776 (1976).

12. D. Proch and H. Schröder, Chem. Phys. Lett. 61, 426 (1979).

13. R. V. Ambartzumian, Yu. A. Gorokhov, V. S. Letokhov, G. N. Makarov and A. A. Puretzky, Zh. Eksp. Teor. Fiz. (Russian) 71, 440 (1976).

14. R. V. Ambartzumian, I. N. Knyazev, V. V. Lobko, G. N. Makarov and A. A. Puretzky, Appl. Phys. 19, 75 (1979).

15. S. S. Alimpiev, N. V. Karlov, B. G. Sartakov and E. M. Khokhlov, Optics Commun. 26, 45 (1978).

16. R. V. Ambartzumian, G. N. Makarov and A. A. Puretzky, Optics Commun. 34, 81 (1980). 
17. K. H. Leary, J. L. Lyman, L. B. Asprey and S. M. Freund, J. Chem. Phys. 68, 1671 (1978).

18. J. L. Lyman, R. C. Anderson, R. A. Fisher and B. J. Feldman, Optics Lett. 3, 238 (1978).

19. J. L. Lyman, W. C. Danen, A. C. Nilsson and A. V. Novak, J. Chem. Phys. 71, 1206 (1979).

20. O. P. Judd, J. Chem. Phys. 71, 4515 (1979).

21. V. V. Lobko, Ph.D. Dissertation, Institute of Spectroscopy Acad. Sci., USSR, Moscow (1979) (Russian).

22. P. Kolodner, C. Winterfeld and E. Yablonovitch, Optics Commun. 20, 119 (1979).

23. I. N. Knyazev, N. P. Kuzmina, V. S. Letokhov, V. V. Lobko and A. A. Sarkisian, Appl. Phys. 22, 429 (1980).

24. S. S. Alimpiev, N. V. Karlov, B. G. Sartakov, S. M. Nikiforov, V. M. Orlovsky, A. M. Prokhorov, E. M. Khokhlov and A. L. Shtarkov, Pis'ma Zh. Eksp. Teor. Fiz. (Russian) 30, 279 (1979).

25. D. O. Ham and M. Rotshild, Optics Lett. 1, 28 (1977).

26. S. S. Alimpiev, Izv. Akad. Nauk SSSR (Russian) 45, 1070 (1981).

27. W. Tsay, C. Riley and D. O. Ham, J. Chem. Phys. 70, 3358 (1979).

28. V. Yu. Baranov, E. P. Velikhov, Yu. R. Kolomiisky, V. S. Letokhov, V. G. Niz'ev, V. D. Pismenny and E. A. Ryabov, Kvantovaja Electron. (Russian) 6, 1062 (1979).

29. R. F. Barrov, T. C. Clark, J. A. Coxon and K. K. Yee, J. Mol. Spectrosc. 51, 428 (1974).

30. J. I. Steinfeld and P. Houston, in: Laser and Coherence Spectroscopy (Plenum Press, New York, London, 1980).

31. P. F. Moulton and A. Mooradian, in: Laser Induced Processes in Molecules, eds. K. L. Kompa and S. D. Smith, Springer Series in Chemical Physics, Vol. 6 (Springer Verlag, Berlin, Heidelberg, New York, 1979) p. 37.

32. P. F. Moulton, D. M. Larsen, J. N. Walpole and A. Mooradian, Optics Lett. 1, 57 (1977).

33. J. I. Steinfeld, C. C. Jensen, T. G. Anderson and Ch. Reiser, Laser Spectroscopy $I V$, eds. H. Walther, K. W. Rothe, Springer Series in Optical Sciences, Vol. 21 (Berlin, Heidelberg, New York, Springer-Verlag, 1979) p. 319.

34. V. N. Bagratashvili, V. S. Doljikov and V. S. Letokhov, Zh. Eksp. Teor. Fiz. (Russian) 76, 18 (1979).

35. J. I. Steinfeld, J. Burak, D. G. Sutton and A. V. Novak, J. Chem. Phys. 52, 5421 (1970).

36. S. A. Akhmanov, V. M. Gordienko, A. V. Mikheenko and V. Ya. Panchenko, Pis'ma Zh. Eksp. Teor. Fiz. (Russian) 26, 603 (1977).

37. D. S. Frankel, Jr., J. Chem. Phys. 65, 1696 (1976).

38. S. S. Alimpiev, V. N. Bagratashvili, N. V. Karlov, V. S. Letokhov, V. V. Lobko, A. A. Makarov, B. G. Sartakov and E. M. Khokhlov, Pis'ma Zh. Eksp. Teor. Fiz. (Russian) 25, 582 (1977).

39. W. Fuss, J. Hartman and W. E. Schmidt, Appl. Phys. 15, 297 (1978).

40. T. F. Deutch and S. R. J. Brueck, Chem. Phys. Lett. 54, 258 (1978).

41. H. Statfast, W. E. Schmidt and K. L. Kompa, Optics Commun. 21, 121 (1977).

42. T. F. Deutch and S. R. J. Brueck, J. Chem. Phys. 70, 2063 (1979).

43. H. S. Kwok and E. Yablonovitch, Phys. Rev. Lett. 41, 745 (1978).

44. A. V. Novak and J. L. Lyman, J. Quant. Spectr. Rad. Transf. 15, 1945 (1975).

45. J. L. Lyman, L. J. Radziemski and A. C. Nilsson, J. Quant. Electr. QE-16, 1174 (1980).

46. V. D. Klimov, V. A. Kuzmenko and V. A. Legasov, Zh. Prikl. Spectr. (Russian) 31, 725 (1979). 
47. A. S. Akhmanov, V. N. Bagratashvili, V. Yu. Baranov, Yu. R. Kolomiisky, V. S. Letokhov, V. D. Pismenny and E. A. Ryabov, Optics Commun. 23, 357 (1977).

48. G. P. Quigley, J. Opt. Soc. Am. 68, 164 (1978).

49. V. A. Apatin, V. M. Krivtzun, Yu. A. Kuritzyn, G. N. Makarov, I. Pak, I. I. Zasavitsky and A. P. Shotov, Pis'ma Zh. Eksp. Teor. Fiz. (Russian) 38, 365 (1983).

50. R. V. Ambartzumian, G. N. Makarov and A. A. Puretzky, Pis'ma Zh. Eksp. Teor. Fiz. (Russian) 28, 246 (1978).

51. R. V. Ambartzumian, Yu. A. Gorokhov, V. S. Letokhov and G. N. Makarov, Pis'ma Zh. Eksp. Teor. Fiz. (Russian) 22, 96 (1975).

52. I. N. Knyazev, Yu. A. Kudryavtsev, N. P. Kuzmina and V. S. Letokhov, $Z h$. Eksp. Teor. Fiz. (Russian) 76, 1281 (1979).

53. R. V. Ambartzumian, V. S. Letokhov, G. N. Makarov and A. A. Puretzky, Pis'ma Zh. Eksp. Teor. Fiz. (Russian) 17, 91 (1973).

54. Aa. S. Sudbo, P. A. Schulz, D. J. Krajnovich, Y. Y. Lee and Y. R. Shen, Optics Lett. 4, 219 (1979).

55. I. N. Knyazev, Yu. A. Kudryavtsev, N. P. Kuzmina and V. S. Letokhov, $Z h$. Eksp. Teor. Fiz. 74, 2017 (1978).

56. R. V. Ambartzumian, V. S. Letokhov, G. N. Makarov and A. A. Puretzky, $Z h$. Eksp. Teor. Fiz. (Russian) 68, 1936 (1975).

57. Yu. A. Kudryavtsev, Kvantovaya Electron. (Russian) 7, 1985 (1980).

58. J. L. Lyman, D. Feldmann, J. Laukemper and K. H. Welge, J. Chem. Phys. 74, 6817 (1981).

59. V. N. Bagratashvili, Yu. G. Vainer, V. S. Doljikov, S. F. Koliakov, A. A. Makarov, L. P. Malyavkin, E. A. Ryabov, E. G. Silkis and V. D. Titov, Pis'ma Zh. Eksp. Teor. Fiz. (Russian) 30, 502 (1979).

60. V. N. Bagratashvili, Y.u. G. Vainer, V. S. Doljikov, S. F. Koliakov, A. A. Makarov, L. P. Malyavkin, E. A. Ryabov, E. G. Silkis and V. D. Titov, Appl. Phys. 22, 101 (1980).

61. V. N. Bagratashvili, V. S. Doljikov, V. S. Letokhov, A. A. Makarov, L. P. Malyavkin, E. A. Ryabov, E. E. Silkis and Yu. G. Vainer, Optics Lett. 6, 148 (1981).

62. (a) J. W. Nilber and G. V. Knighten, in: Topics in Current Physics, ed. A. Weber (Springer, Berlin, 1979); (b) M. D. Levenson and J. J. Song, in: Topics in Current Physics, Vol. 21, ed. M. Feld and V. S. Letokhov (Springer, Berlin, 1980) p. 293.

63. R. V. Ambartzumian, S. A. Akhmanov, A. M. Brodnikovsky, S. M. Gladkov, A. V. Evseev, V. N. Zadkov, M. G. Karimov, M. I. Koroteev and A. A. Puretzky, Pis'ma Zh. Eksp. Teor. Fiz. (Russian) 35, 170 (1982).

64. S. S. Alimpiev, S. I. Valjansky, S. H. Nikiforov, V. V. Smirnov, B. G. Sartakov, V. I. Fabelinsky and A. L. Shtarkov, Pis'ma Zh. Eksp. Teor. Fiz. (Russian) 35, 291 (1982).

65. J. F. Bott, Appl. Phys. Lett. 32, 624 (1978).

66. J. Hudgens and J. D. McDonald, J. Chem. Phys. 76, 173 (1982).

67. D. S. Frankel, Jr. and T. J. Manuccia, Chem. Phys. Lett. 54, 451 (1978).

68. R. E. McNair, S. F. Fulghum, G. W. Flynn, M. C. Feld and B. J. Feldman, Chem. Phys. Lett. 48, 241 (1977).

69. V. S. Letokhov, A. A. Makarov and E. A. Ryabov, Dokl. Akad. Nauk. (Russian) 212, 75 (1973).

70. G. P. Quigley, Optics Lett. 3, 106 (1978).

71. L. P. Herman and J. B. Marling, Chem. Phys. Lett. 64, 75 (1979).

72. N. V. Chekalin, V. S. Letokhov, V. N. Lokhman and A. N. Shibanov, Chem. Phys. 36, 415 (1979). 
73. J. C. Stephenson, D. S. King, M. F. Goodman and J. Stone, J. Chem. Phys. 70, 4496 (1979).

74. J. L. Lyman, R. C. Anderson, R. A. Fisher and B. J. Feldman, Chem. Phys. 45, $325(1980)$.

75. V. S. Doljikov, Yu. R. Kolomiisky and E. A. Ryabov, Chem. Phys. Lett. 80, 433 (1981).

76. V. N. Bagratashvili, V. S. Doljikov, V. S. Letokhov, A. A. Makarov, E. A. Ryabov and V. V. Tyacht, Zh. Eksp. Teor. Fiz. (Russian) 77, 2238 (1979) [Sov. Phys.JETP 50, 1075 (1979)].

77. V. N. Bagratashvili, V. S. Doljikov, V. S. Letokhov and E. A. Ryabov, Laser Induced Processes in Molecules, eds. K. L. Kompa and S. D. Smith, Springer Series in Chemical Physics, Vol. 6, Springer-Verlag (Berlin, Heidelberg, New York, 1979) p. 179.

78. C. R. Quick, Jr. and C. Wittig, J. Chem. Phys. 69, 4202 (1978).

79. F. M. Lussier, J. I. Steinfeld and T. F. Deutch, Chem. Phys. Lett. 58, 277 (1978).

80. J. Stone, E. Thiele, M. F. Goodman, J. C. Stephenson and D. S. King, J. Chem. Phys. 73, 2259 (1980).

81. V. N. Bagratashvili, V. N. Bourimov, M. V. Kuzmin, V. S. Letokhov and A. P. Sviridov, Laser Chemistry 1, 133 (1983).

82. R. G. Harrison, H. L. Hawkins, R. M. Leo and P. John, Chem. Phys. Lett. 70, 555 (1980).

83. C. N. Plum and P. L. Houston, Appl. Phys. 24, 143 (1982).

84. M. Rotchschild, W. S. Tsay and D. O. Ham, Optics. Commun. 24, 237 (1978).

85. R. V. Ambartzumian, Yu. A. Gorokhov, G. N. Makarov, A. A. Puretzky and N. P. Furzikov, Chem. Phys. Lett. 45, 231 (1977).

86. B. I. Vasiljev, N. A. Vyshujakov, V. T. Galochkin, A. Z. Grasjuk, A. N. Dyad'kin, A. K. Zhigalkin, V. A. Kovalevsky, V. N. Kosinov, A. H. Oraevsky and A. N. Surhanov, Pis'ma Zh. Eksp. Teor. Fiz. (Russian) 30, 29 (1979).

87. O. N. Avatkov, V. N. Bagratashvili, I. N. Knyazev, Yu. R. Kolomijsky, V. S. Letokhov, V. V. Lobko and E. A. Ryabov, Kvantovaya Electron. (Russian) 4, 741 (1977).

88. O. N. Avatkov, E. B. Aslanidi, A. B. Bakhtadze, R. I. Zainullin and Yu. S. Turitshev, Kvantovaya Electron. (Russian) 6, 388 (1979).

89. G. A. Askarian, Zh. Eksp. Teor. Fiz. (Russian) 48, 666 (1965).

90. M. V. Kuz'min, Kvantovaya Electron. (Russian) 5, 759 (1978).

91. V. I. Arnol'd Mathematical Methods of Classical Mechanics, (Nauka, Moscow, 1974) (in Russian).

92. N. N. Bogoljubov and Yu. A. Mitropol'sky, Asymptotical Methods in the theory of Nonlinear Oscillations (Russian) (Nauka, Moscows, 1974).

93. V. I. Gortchakov and V. N. Sazonov, Zh. Eksp. Teor. Fiz. (Russian) 70, 467 (1976).

94. M. V. Fedorov, Zh. Eksp. Teor. Fiz. (Russian) 73, 134 (1977).

95. R. V. Ambartzumian, Yu. A. Gorokhov, V. S. Letokhov, G. N. Makarov and A. A. Puretzky, Pis'ma Zh. Eksp. Teor. Fiz. (Russian) 23, 26 (1976).

96. D. M. Larsen and N. Bloembergen, Optics. Commun. 17, 254 (1976).

97. D. M. Larsen, Optics Commun. 19, 404 (1976).

98. V. M. Akulin, S. S. Alimpiev, N. V. Karlov and B. G. Sartakov, Zh. Eksp. Teor. Fiz. (Russian) 72, 88 (1977).

99. V. T. Platonenko, Pis'ma Zh. Eksp. Teor. Fiz. (Russian) 25, 52 (1977).

100. C. D. Cantrell and H. W. Galbraith, Optics. Commun. 21, 374 (1977).

101. T. Hecht, J. Mol. Spectr. 5, 355 (1960).

102. K. Fox, Optics Lett. 1, 214 (1977). 
103. J. R. Acherhalt and H. W. Galbraith, J. Chem. Phys. 69, 1200 (1978).

104. C. D. Cantrell and K. Fox, Optics Lett. 2, 151 (1978).

105. H. W. Galbraith and J. R. Acherhalt, Optics Lett. 3, 109 (1978).

106. S. Bittenson and P. L. Houston, J. Chem. Phys. 67, 4819 (1977).

107. E. Würzberg, L. J. Kovalenko and P. L. Houston, J. Chem. Phys. 67, 2017 (1977).

108. G. J. Pert, IEEE J. Quant. Electr. QE-9, 435 (1973).

109. M. V. Kuz'min and V. N. Sazonov, Zh. Eksp. Teor. Fiz. (Russian) 73, 422 (1977).

110. V. S. Letokhov and A. A. Makarov, Optics Commun. 17, 250 (1976).

111. V. S. Letokhov and A. A. Makarov, Appl. Phys. 16, 47 (1978).

112. E. S. Medvedev, Chem. Phys. 41, 103 (1979).

113. M. V. Kyz'min and V. N. Sazonov, Kvantovaya Electron. (Russian) 6, 539 (1979).

114. V. M. Agranovitch and V. I. Rupasov, Preprint of the Institute of Spectroscopy USSR Acad. Sci., Moscow, 11/32 (1976).

115. D. P. Hodkinson and J. S. Briggs, J. Phys. B: Atom. Mol. Phys. 10, 2583 (1977).

116. M. Tamir and R. D. Levine, Chem. Phys. Lett. 46, 208 (1977).

117. H. B. Bebb and A. Gold. Phys. Rev. 143, 1 (1966).

118. F. H. Faisal, Optics Comm. 17, 247 (1976).

119. S. Mukamel and J. Jortner, Chem. Phys. Lett. 40, 150 (1976).

120. V. N. Sazonov and V. Yu. Finkelstein, Zh. Eksp. Teor. Fiz. (Russian) 73, 1306 (1977).

121. V. R. Block, G. M. Krochick and Yu. G. Khronopulo, Zh. Eksp. Teor. Fiz. (Russian) 76, 46 (1979).

122. W. L. Barnes, J. Susskind, R. N. Hunt and E. K. Piyler, J. Chem. Phys. 56, 5160 (1972).

123. L. W. Pinkley, K. N. Rao, M. Dang-Nhu and G. Poussique, J. Mol. Spectr. 63, 402 (1976).

124. I. N. Knyazev and V. V. Lobko, Izv. Akad. Nauk SSSR (Russian) 43, 385 (1979).

125. I. N. Knyazev and V. V. Lobko, Zh. Eksp. Teor. Fiz. (Russian) 77, 816 (1979).

126. I. N. Knyazev, V. S. Letokhov and V. V. Lobko, Optics Commun. 25, 337 (1978).

127. M. R. Aliev and V. M. Mikhailov, Pis'ma Zh. Eksp. Teor. Fiz. (Russian) 30, 300 (1979).

128. J. Moret-Bailly, J. Mol. Spectr. 15, 344 (1965).

129. V. M. Akulin, Zh. Eksp. Teor. Fiz. (Russian) 76, 1933 (1979).

\section{MULTIPLE-PHOTON EXCITATION OF MOLECULES IN VIBRATIONAL QUASICONTINUUM}

Many points dealing with excitation of vibrational quasicontinuum have been discussed in Chapters 2 and 3 (Refs. 1,2). In this chapter in Section 6.1 consideration is given primarily to the basic results of experimental research of MP molecular excitation in the region of quasicontinuum. In Section 6.2 the theory of MP excitation in the quasicontinuum will be correlated with experiment. And, finally, in Section 6.3 we shall discuss the vibrational energy distribution. 


\subsection{Experimental study of MP excitation of molecules in the quasi- continuum}

Let us consider the most important characteristics of the process of MP molecular excitation in the region of quasicontinuum obtained with the use of the various experimental methods.

\subsubsection{Average level of excitation in the quasicontinuum}

The methods of absorbed energy calorimetry given in Chapter 5 make it possible to measure the average energy absorbed by all the molecules in the volume under irradiation $\bar{\varepsilon}$. However, not all the molecules but just some fraction of them $q$ is involved in the process of MP excitation. Moreover, the molecules get divided distinctly into two ensembles: "cold" molecules remaining at lower discrete vibrational levels and "hot" molecules excited to higher vibrational states in the quasicontinuum (see Section 5.3) It enables to determine average level of excitation of molecules in quasicontinuum.

$$
\bar{\varepsilon}_{q}=\bar{\varepsilon} / q
$$

where $\bar{\varepsilon}$ and $q$ are experimentally measurable values.

Figure 6.1 presents the dependences of the average excitation level of $\mathrm{SF}_{6}, \mathrm{CF}_{3} \mathrm{I}$ and $\mathrm{OsO}_{4}$ molecules in the quasicontinuum $\bar{\varepsilon}_{q}$ on IR laser energy fluence $\Phi$. These dependences were obtained in Section

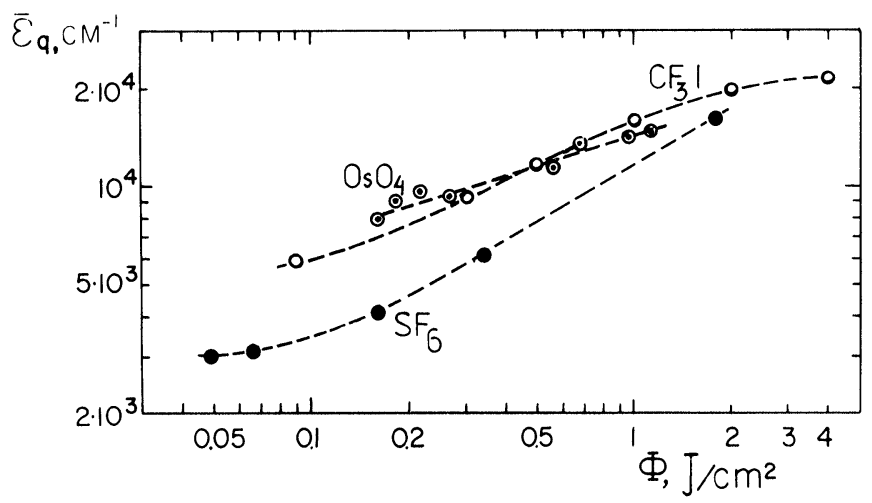

FIGURE 6.1 Dependences of the average level of excitation of $\mathrm{CF}_{3} \mathrm{I}, \mathrm{OsO}_{4}$ and $\mathrm{SF}_{6}$ molecules on radiation energy fluence $\Phi$. (From Section 5, Refs. 50 and 76). 
5(ref. 50, 76) from independent measurements of $\bar{\varepsilon}$ and $q$. With the radiation energy fluence over $0.2 \mathrm{~J} / \mathrm{cm}^{2}$, the value of $\bar{\varepsilon}_{q}$ increases monotonously at first and then (for $\mathrm{CF}_{3} \mathrm{I}$ at least) begins to be saturated. This saturation at high $\Phi$ for $\mathrm{CF}_{3} \mathrm{I}$ is explained by molecular dissociation during a pulse. Indeed, the value $\bar{\varepsilon}_{q}^{\max } \simeq 21000 \mathrm{~cm}^{-1}$ exceeds just a little the dissociation energy of the $\mathrm{CF}_{3} \mathrm{I}$ molecule $D \simeq 19000 \mathrm{~cm}^{-1}$. So little overexcitation of the $\mathrm{CF}_{3} \mathrm{I}$ molecule (no more than 1 or 2 laser quanta) is caused, first of all, by a high rate of unimolecular dissociation due to a small number of atoms in it (see Section 3, ref. 1, Section 4). Besides, the resulting $\mathrm{CF}_{3}$ radical does not make a substantial contribution to MP absorption (Section 5, Ref. 76), and so calorimetric measurements even at effective dissociation of $\mathrm{CF}_{3} \mathrm{I}$ can give information on its average excitation level.

A quite different situation can be realized for rather complex molecules. First of all, in such molecules considerable excitation over the dissociation limit can take place during an IR pulse due to a small rate of unimolecular dissociation. For the $\left(\mathrm{CF}_{3}\right)_{3} \mathrm{CBr}$ molecule, for example, during $\tau_{p}=10^{-6} \mathrm{~s}$ the dissociation energy can be exceeded twice (Section 5, Ref. 81). The radicals formed in MP dissociation of complex molecules can also often absorb effectively IR radiation energy. It presents a serious problem in direct determination of the $\bar{\varepsilon}_{q}$ value since it is difficult to discriminate in calorimetric experiments the MP absorption by original molecules from that by dissociation products. More precise quantitative data of this kind can be obtained from experiments with molecular beams.

Let us consider the effect of IR radiation intensity on the MP excitation of molecules in the vibrational quasicontinuum. Figure 6.2 shows how the average number of absorbed quanta $\bar{n}$ for the $\mathrm{SF}_{6}$ molecule depends on the IR radiation intensity $I$ at fixed energy fluence $\Phi=0.2 \mathrm{~J} / \mathrm{cm}^{2} .{ }^{1}$ The value of $I$ was varied by varying the $\mathrm{CO}_{2}$ laser pulse duration $\tau_{p}$ from standard $\left(\tau_{p}=0.1 \mu \mathrm{s}\right)$ to ultrashort $\left(\tau_{p}=\right.$ $30 \mathrm{ps})$. As the laser intensity is increased by about 4 orders, the value of $\bar{n}$ increases by one and a half orders, the most intense increase observed at high values of $I$ (in a range $10^{9}-10^{10} \mathrm{~W} / \mathrm{cm}^{2}$ ).

In the region of low intensities $I=10^{6}-10^{7} \mathrm{~W} / \mathrm{cm}^{2}$, when $\bar{n}<4$ quanta per molecule, the fraction of molecules involved in the process of MP excitation $q<1$ (see 5.3.3). In this case the increase of $\bar{n}$ can be naturally attributed to the power broadening effects at discrete vibrational levels, which cause $q$ and hence $\bar{n}$ to increase. At the same 


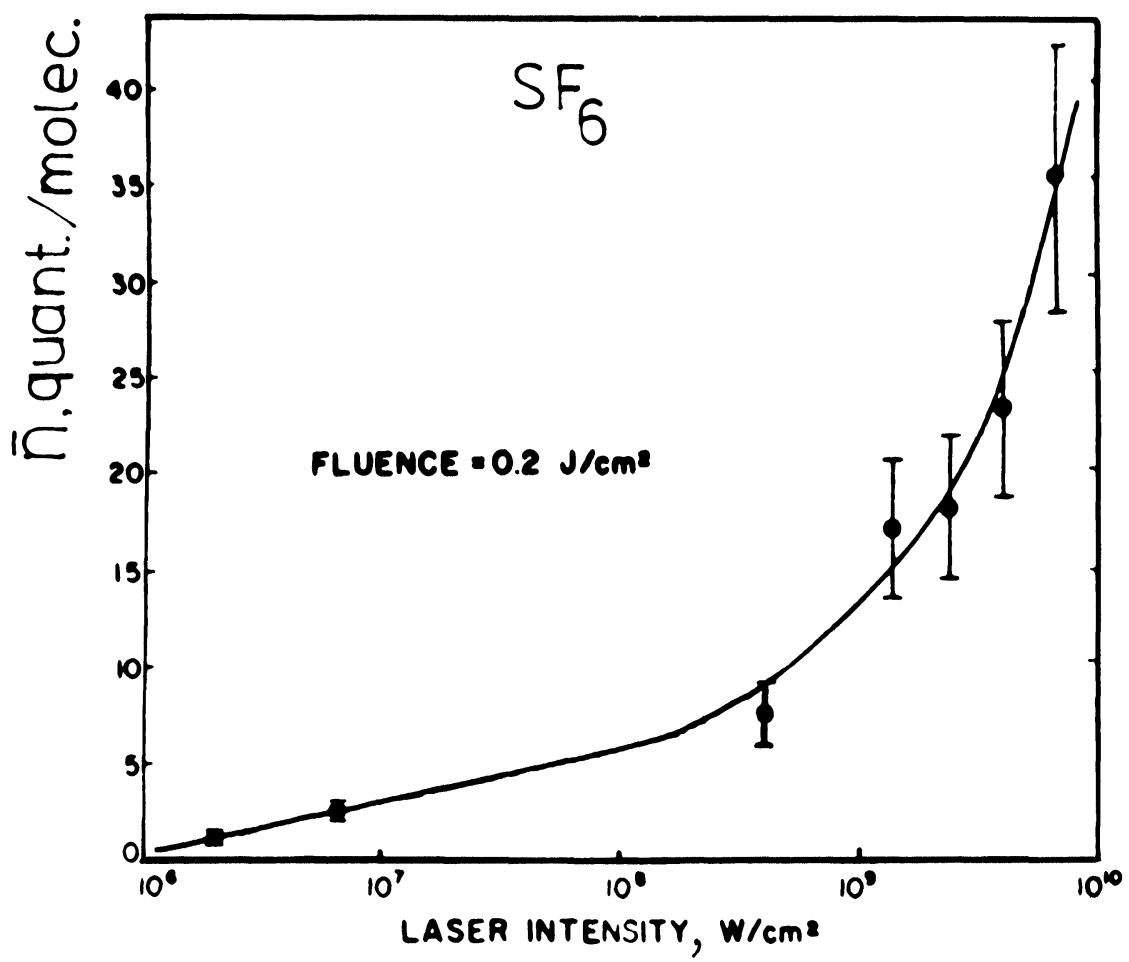

FIGURE 6.2 Dependence of the average number of absorbed quanta in $\mathrm{SF}_{6}$ on IR radiation intensity (From Ref. 1).

time at high radiation intensities $I>10^{9} \mathrm{~W} / \mathrm{cm}^{2}$ and $\Phi=0.2 \mathrm{~J} / \mathrm{cm}^{2}$ the power broadening effects provide excitation of almost all $\mathrm{SF}_{6}$ molecules to the quasicontinuum $q \simeq 1$. In this case, since $\bar{n}_{q}=\bar{n}$, with an increase of $I$ the excitation level of $\mathrm{SF}_{6}$ to the quasicontinuum $n_{q}$ increases. It must be said that such an increase comes about mainly with $I>10^{9} \mathrm{~W} / \mathrm{cm}^{2}$, i.e., under MP excitation by supershort $\left(10^{-9}+\right.$ $10^{-11} \mathrm{~s}$ ) pulses. Really, as many experiments show in which MP absorption and dissociation are studied (see ref. 2, 3, and Section 5, ref. 9), with $I>10^{9} \mathrm{~W} / \mathrm{cm}^{2}$ the level of excitation of molecules in the quasicontinuum is slightly subjected to intensity and depends mainly on laser energy fluence $\Phi$. The authors ${ }^{1}$ explain the increase of absorbed energy with increasing $I$ by the change-over from incoherent excitation of $\mathrm{SF}_{6}$ molecule in the quasicontinuum to coherent excitation. 


\subsubsection{Stochastization of vibrational energy in MP excitation of molecules}

The internal energy distribution of a MP excited molecule over its vibrational degrees of freedom is one of important and very likely, one of the most complex questions for research.

At an early stage of research of MP processes in molecules many investigators hoped for mode (or bond)-selective excitation of polyatomic molecules and associated photochemical reactions. It should be noted that the authors of the first papers in this field pointed to inevitable excitation of many vibrational modes due to the transitions in the vibrational quasicontinuum. In the works which followed it was shown that the process of strong MP excitation involved all or, at least, many vibrational modes of polyatomic molecules. This refers to a great majority of cases under typical experimental conditions with the duration of IR radiation pulses $\tau_{p}=10^{-6}-10^{-8} \mathrm{~s}$. In other words, at sufficiently strong MP excitation of molecules the vibrational molecular energy is subjected to (complete or partial) stochastization. In Section 2.3.3 consideration was given to potential mechanisms of stochastization of molecular vibrational motion. Here we want to consider some experimental approaches to this problem. As the studies into MP dissociation of various molecules show, the statistical theory of unimolecular dissociation (RRKM theory ${ }^{4}$ ) agrees well, as a rule, with experimental results (at least for not very large molecules, such as $\mathrm{SF}_{6}, \mathrm{CF}_{3} \mathrm{I}, \mathrm{CF}_{2} \mathrm{CFCl}$ and others). This means that the vibrational motion near the dissociation limit for such molecules is stochastized and it can be described on the basis of statistical methods.

Search of nonstochasticity There are some experiments when in their interpretation a deflection of the internal energy distribution from the statistically equilibrium one is permitted.

In Ref. 5 the chemical processes induced by exciting the vibration of $\mathrm{CH}$-bond and the deformation vibration of $\mathrm{CH}_{2}$-bond in cyclopropane were compared. Two primary reactions are essential in this case. One of them is an isomerization reaction:<smiles>C/C=C\C</smiles> 
and the other one is a fragmentation reaction:<smiles>C=CCCC</smiles>

Fragmentation reaction (6.3) has a far higher energy barrier and in case of statistical energy distribution over modes must not play any appreciable part.

The $\mathrm{CH}$-bond of cyclopropane at $3106 \mathrm{~cm}^{-1}$ was excited by a high-energy pulse of a powerful optical parametric oscillator and the $\mathrm{CH}_{2}$-bond at $1080 \mathrm{~cm}^{-1}$ was excited by a pulse of a TEA $\mathrm{CO}_{2}$ laser. The reaction products were analyzed with a gas chromatograph. In exciting the $\mathrm{CH}$-bond at the $3.22 \mu \mathrm{m}$ wavelength one could observe isomerization reaction (6.2) with the lowest energy barrier. The collisions of excited molecules with the buffer gas (argon) gave birth to fragmentation products with a small yield. In exciting the $\mathrm{CH}_{2}$-bond at $9.5 \mu \mathrm{m}$ at low buffer gas pressures one could observe a preferential fragmentation reaction that continued making an appreciable contribution even at higher buffer gas pressures. Basing on these results the authors concluded that the reaction product distribution depended on which of the vibrational modes was under excitation. However, this conclusion, to our opinion, cannot be rigorously proved without measuring the energies absorbed by a molecule in each case.

In refs. 6 and 7 the MP dissociation of rather a complex volatile uranium compound $\mathrm{UO}_{2}$ (hfacac) ${ }_{2}$ THF by the radiation of a $\mathrm{CO}_{2}$ laser under continuous and pulsed operation was studied. Photodissociation was done in a molecular beam when collisions and subsequenet thermal heating could be avoided even under continuous irradiation. The $\mathrm{CO}_{2}$ laser radiation was in resonance with the asymmetrical vibration of the uranil group which is sensitive to the isotope composition of uranium and oxygen. In Ref. 7 they could observe the MP dissociation of this molecular compounds by pulsed $\mathrm{CO}_{2}$ laser radiation. MP absorption and dissociation of such a molecule belong to the case of molecules with a very low vibrational quasicontinuum limit considered in section 5, ref. 1 (Chapter 1). A high dissociation yield can be achieved at extremely low pulse energy fluence $\left(0.1 \mathrm{~J} / \mathrm{cm}^{2}\right)$. In the theoretical model of MP absorption and MP dissociation developed in Ref. 7 it is assumed that the absorbed energy is distributed only among a part of vibrational modes. It is obvious that direct experiments 
with the use of shorter (of the order of $1 \mathrm{~ns}$ and shorter) $\mathrm{CO}_{2}$ laser pulses are required to prove this conclusion.

In Ref. 86 of Chapter 5 the dissociation yields of the $\mathrm{CCl}_{4}$ molecule were correlated as the fundamental vibration $\nu_{3}$ was excited by $\mathrm{NH}_{3}$ laser pulses at $771 \mathrm{~cm}^{-1}$ and the compound mode $\nu_{1}+\nu_{2}+\nu_{4}$ by $\mathrm{CO}_{2}$ laser pulses at $980 \mathrm{~cm}^{-1}$. In this work the opto-acoustic method was applied to measure the energy absorbed per one molecule in the volume under irradiation. On the basis of different dissociation yields $\beta$ at the same absorbed energy $\bar{\varepsilon}$ a conclusion was drawn on the presence of "mode-selectivity" during MP dissociation of $\mathrm{CCl}_{4}$ by IR radiation. This conclusion, however, cannot be considered proved without reliable measurement of the fraction of molecules $q$ involved in the process of MP dissociation in every case. If, for example, the fraction of excited molecules during excitation of different bands differ, which seems quite natural, the real excitation energy level of $\mathrm{CCl}_{4}$ molecules is different which can result in different dissociation yields.

In Ref. 26 of Chapter 5 the two-frequency excitation of the $\mathrm{SF}_{6}$ molecule was studied in detail. The MP absorption was measured through direct and opto-acoustic calorimetry. The MP dissociation yield of $\mathrm{SF}_{6}\left(\mathrm{SF}_{6} \rightarrow \mathrm{SF}_{5}+\mathrm{F}\right)$ was determined from the IR luminescence intensity of the vibrationally excited HF molecule $(\lambda=2.5 \mu \mathrm{m})$ resulting from the reaction of atomic fluorine with $\mathrm{H}_{2}$ scavenger. The values of absorbed energy from the second IR field $\left(\Omega_{2}\right)$ and dissociation yield $\beta$ were measured at a fixed frequency of the first IR field. Figure 6.3 shows the dependence of $\bar{\varepsilon}_{2}, \beta$ and the dissociation efficiency $\varphi_{2} \sim \beta / \bar{\varepsilon}$ on $\Omega_{2}$. As $\Omega_{2}$ shifts to the long-wave side, the MP dissociation efficiency increases greatly (by 50 times as varies between 950 and $790 \mathrm{~cm}^{-1}$ ). This result in Ref. 26 of Chapter 5 is explained by a different degree of stochastization, i.e., by different intermode distribution in $\mathrm{SF}_{6}$ during MP excitation at different frequencies. But it can be also explained alternatively, i.e., by a different fraction of molecules preexcited by the first field $\Omega_{1}$, interacting with the second field at different $\Omega_{2}$.

Indirect observation of stochastization There are several experimental works the authors of which report that they have managed to study the effect of vibrational energy stochastization. In Refs. 37,42 and 43 of Chapter 5, for example, the experiment was identical to the experiments on double IR-IR resonance described in Section 


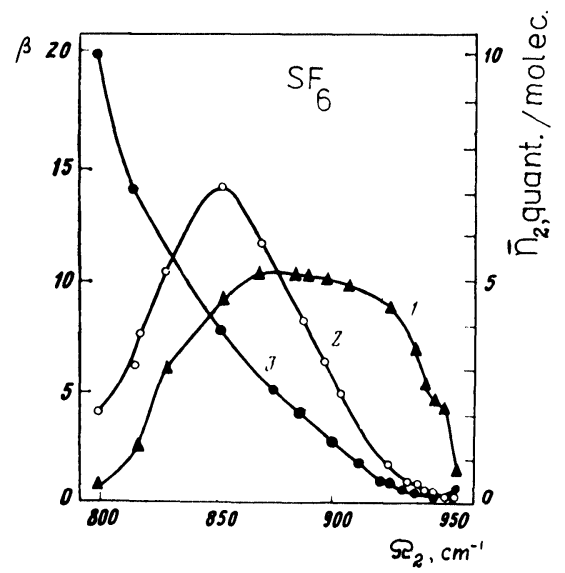

FIGURE 6.3 Dependences of the average absorbed energy $\bar{n}_{2}$ (curve 1), dissociation yield $\beta$ (curve 2) and its quantum efficiency (in relative units) (curve 3) on the frequency of the second field $\Omega_{2}$ for the $\mathrm{SF}_{6}$ molecules preexcited by $\Omega_{1}$ field. $\Omega_{1}=944.2 \mathrm{~cm}^{-1}$, $\phi_{1}=3 \mathrm{~J} / \mathrm{cm}^{2}$. (From Section 5, Ref. 26).

5.2.1. Gaseous $\mathrm{SF}_{6}$ was excited by a laser pulse, and the probe laser beam absorption was measured both during a pulse and after it. The changes in the signal of probe beam transmission were interpreted as a process of energy transfer from the molecular mode under excitation to the rest of vibrational degrees of freedom. In Ref. 37 of Chapter 5 one could observe strong changes in the signal at the rear edge of exciting laser pulse, its duration being $\tau_{p}=2 \mathrm{~ns}$. These changes in the signal, however, cannot be related in any way either to the process of collisional vibrational relaxation or the process of "collisionless" relaxation. Really, as it has been vividly demonstrated in Ref. 32 of Chapter 5, a similar effect of changes in the transmission signal always takes place and is connected with the shift of the transition probed due to the dynamic Stark effect.

In Ref. 42 of Chapter 5 the changes in the absorption spectrum of excited $\mathrm{SF}_{6}$ molecules after the action of a laser pulse with its duration $\tau_{p}=100 \mathrm{~ns}$ were interpreted as a process of vibrational energy stochastization. This spectrum evolution, however, is quite similar to the one illustrated in Figure 5.10. As it follows from the results of Ref. 34 of Chapter 5, this evolution can be easily explained by the process of vibrational energy exchange between the upper and lower molecular ensembles. Therefore, the effect disclosed is hardly probable to be 
related to the process of vibrational energy stochastization (see, also, Section 5, Ref. 43).

In Section 5, Ref. 43 changes in the absorption of excited molecules were disclosed, on a much shorter time scale though. In this work the exciting laser pulse duration was $\tau_{p}=10 \mathrm{ps}$. Such a pulse duration, in principle, does not make it impossible to observe the effect of vibrational energy stochastization in the $\mathrm{SF}_{6}$ molecule.

To our opinion, however, one should carefully approach the quantitative interpretation of any observation of vibrational energy stochastization by the method of double IR-IR resonance. The cause is that the integral absorption in the band concentrated nearby the vibrational mode of molecule active in the IR spectrum must slightly depend on energy and can be related only to anharmonic terms which is not considered at a quantitative level now.

Direct observation of vibrational stochastization The possibility of independent measurement of the vibrational energy stored in different molecular modes is obviously a critical condition for direct experi mental observation of molecular vibration stochastization. In the most of the above-considered experiments the information on intramolecular energy distribution was obtained only indirectly and this made the interpretation of results ambiguous. In Chapter 5, Ref. 1 (Section 2.7) we have already noted that such methods of spectroscopic diagnostics as spontaneous Raman scattering and IR fluorescence can be used for direct measurement of the vibrational energy distribution in MP excited molecules (see, also, Section 5.2). As it is shown in Section 5, Ref. 1 the integral intensity of anti-Stokes Raman scattering as well as of IR fluorescence are proportional to the vibrational energy stored in the mode being probed (see relations (5.9) and (5.10)). From this fact two obvious possibilities of study into inframolecular distribution follow. The first of them is connected with observing the time evolution of integral anti-Stokes signal $S^{\text {AS }}$. It is apparent that if Raman probing is performed after excitation, in the $\tau_{d}$ time that materially exceeds the characteristic time of intermode collisional energy exchange, the integral signal $S^{\mathrm{AS}}$ will be proportional to the equilibrium energy in the $\nu_{i}^{\mathrm{RS}}$ mode under probing. ${ }^{8}$ By measuring the dependence $S^{\mathrm{AS}}\left(\tau_{d}\right)$ it is possible to find at a quantitative level to what extent the energy distribution over modes formed in the very process of MP excitation differs from the statistically equilibrium one. 
The characteristic dependences $S^{\mathrm{AS}}\left(\tau_{d}\right)$ for $\mathrm{SF}_{6}$ and $\mathrm{CF}_{3} \mathrm{I}$ molecules ${ }^{8}$ are given in Figure 6.4. Within the limits of experimental error the value of $S^{\mathrm{AS}}$ is independent of $\tau_{d}$ over a range from $0-15 \mathrm{~ns}$ up to several microseconds. At a pressure of 0.1 Torr this corresponds to the change over from the region where the collisional energy exchange can be neglected to the region where this process is essential. The damping of the signal with $\tau_{d}>5 \mu$ s according to Ref. 8 of Chapter 5 is related to the diffusion of molecules from the region of excitation. The results obtained in Ref. 8 make it possible to conclude that under the given conditions of $\mathrm{MP}$ excitation of $\mathrm{SF}_{6}$ and $\mathrm{CF}_{3} \mathrm{I}$ molecules the energy stored in a molecule is not redistributed between its modes, i.e., the distribution is equilibrium or, at least, approaches it.

Here it should be noted that the conclusion drawn is based on the fact that the anti-Stokes signal is independent of time over a wide
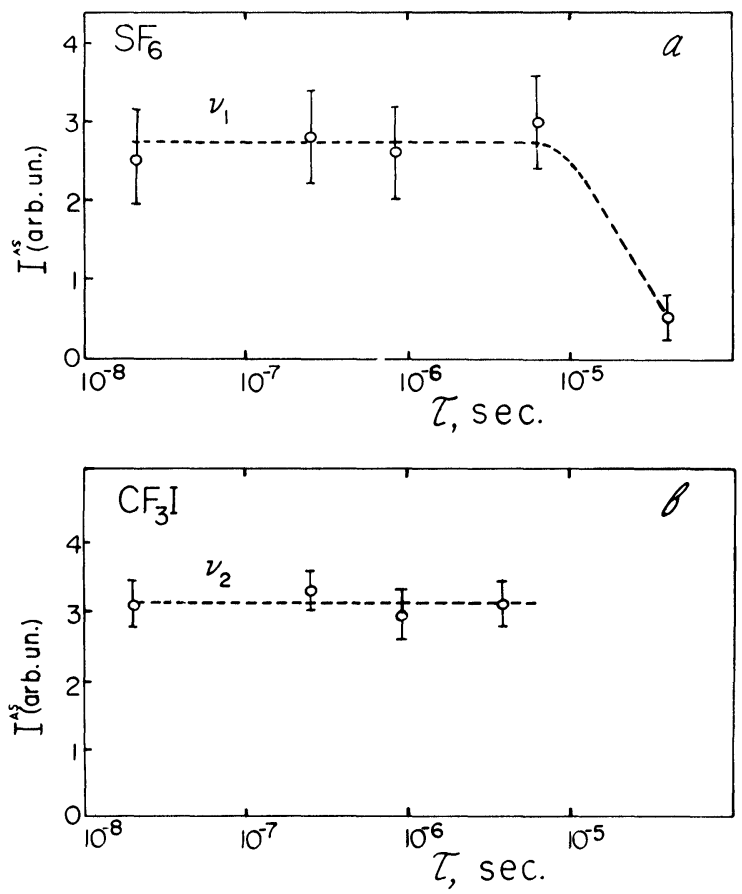

FIGURE 6.4 Dependences of the integral intensity of RS signal in the anti-Stokes region $I^{\mathrm{AS}}$ on the delay time between the exciting (IR) and probing (UV) pulses for $\mathrm{SF}_{6}$ (a) and $\mathrm{CF}_{3} \mathrm{I}$ (b) molecules (From Ref. 8). 
range of values of the $\tau_{p}$ parameter ( $1.5 \mathrm{~ns}$ Torr $-2 \mu \mathrm{s}$. Torr). However, collisions bring about not only equilibrium distribution of molecular internal energy if any was absent. Since during MP excitation of $\mathrm{SF}_{6}$ and $\mathrm{CF}_{3} \mathrm{I}$ molecules there are two ensembles of "cold" and "hot" molecules formed, the average energy of "hot" (excited) molecules during collisions can be reduced so that the quantum character of the heat capacity of vibrational degrees of freedom becomes essential. Because of this the value of energy stored in a mode can vary even if the intermode distribution is equilibrium. In case of $\mathrm{SF}_{6}$ in the above-discussed experiments this effect lies within the limits of measurement accuracy and is not observable. But it imposes certain limits on the potentialities of the technique in use.

The information on the character of intranolecular energy distribution can be also obtained by comparing the results of direct calorimetry of absorbed energy and the results of Raman probing. Such comparison of the dependences $\bar{\varepsilon}=\bar{\varepsilon}(\Phi)$ and $S^{\mathrm{AS}}=S^{\mathrm{AS}}(\Phi)$ has been already performed in Section 5.2.3 under the conditions when the energy distribution in a molecule at the instant of probing is equilibrium due to collisional intermode energy exchange. In accordance with Ref. 8, the value of anti-Stokes signal is proportional to absorbed energy also in case of probing $\mathrm{SF}_{6}$ under the conditions when the intermode exchange during collisions can be neglected ( $p \tau<4$ ns $\cdot$ Torr), i.e., $S_{\text {collisionless }}^{\mathrm{AS}} \sim \bar{\varepsilon}$. Since at the same time $S_{\text {collisional }}^{\mathrm{AS}} \sim S_{\text {collisionless, }}^{\mathrm{AS}}$, this confirms again the fact that the collisions after the IR pulse do not cause changes in the character of distribution of the energy absorbed by a molecule between its vibrational degrees of freedom. Thus, the abovegiven information on Raman probing of MP excited molecules unambiguously points to the fact that under the conditions of the above experiments the MP excitation of $\mathrm{SF}_{6}$ and $\mathrm{CF}_{3} \mathrm{I}$ molecules gives rise to intramolecular distribution with the near-statistically equilibrium distribution of absorbed energy among all or, at least, most modes of these molecules. So the stochastic character of vibrational motion in the molecules concerned is formed in the process of their interaction with the IR radiation.

The results obtained in Ref. 8 make it possible to estimate the stochastization limit. The minimum $\mathrm{CO}_{2}$ laser pulse energy fluence at which the Raman signal could be registered reliably in Ref. 8 (the signal-to-noise ratio was $6: 1$ ) was equal to $0.06 \mathrm{~J} / \mathrm{cm}^{2}$ for $\mathrm{SF}_{6}$ and $0.09 \mathrm{~J} / \mathrm{cm}^{2}$ for $\mathrm{CF}_{3} \mathrm{I}$. From the known values of absorbed energy $\bar{\varepsilon}$ 
and the fraction of molecules involved in the process of MP excitation, according to (6.1), one can find the average absorbed energy for the molecules excited to the upper ensemble $\bar{\varepsilon}_{q}$. If we add the initial average vibrational energy at $293 \mathrm{~K}$ to this value, we can estimate the stochastization limits according to: ${ }^{8}$

$$
\begin{gathered}
\mathrm{SF}_{6} \text { molecule }-E_{\text {stoch }}=3900 \pm 500 \mathrm{~cm}^{-1} \\
\mathrm{CF}_{3} \mathrm{I} \text { molecule }-E_{\text {stoch }}=6000 \pm 500 \mathrm{~cm}^{-1}
\end{gathered}
$$

It should be noted that the values of $E_{\text {stoch }}$ given for $\mathrm{SF}_{6}$ and $\mathrm{CF}_{3} \mathrm{I}$ molecules are the upper estimates which, according to Ref. 8, were possible at the sensitivity level of the system used to record the Raman signal. It should be emphasized here that, as the radiation energy fluence decreases below $\Phi=0.1-0.5 \mathrm{~J} / \mathrm{cm}^{2}$, the absorbed energy $\bar{\varepsilon}$ and hence the value of $S^{\text {AS }}$ are reduced mainly due to a decrease in the fraction of excited molecules $q$ (Figure 6.2). As noted in 6.1.2, the excitation level $\bar{\varepsilon}_{q}$ both for $\mathrm{SF}_{6}$ and $\mathrm{CF}_{3} \mathrm{I}$ decreases here very slowly (Figure 6.2). This means that the transition spectrum changes qualitatively for the molecules involved in the process of MP excitation in the energy ranges given above. The excitation rates of molecules to energies below $E_{\text {stoch }}$ are at least several times higher than the rates of their further excitation to the energy range $E>E_{\text {stoch }}$. This corresponds exactly to the vibrational energy stochastization limit (or the quasicontinuum) near which the structure of real wave functions is varied (Ref. 1, Section 2.3). In the region $E>E_{\text {stoch }}$ the wave functions of the states of the mode interacting with the laser radiation on account of intermode resonances mix with many other wave functions. The potential mechanisms of molecular vibration stochastization are considered in Chapter 5, Ref. 1 (Section 2.3.3).

We have considered a number of experimental data about intramolecular distribution during MP excitation of molecules or, to be more precise, stochastization of molecular vibrational energy. This question is of great interest for molecular spectroscopy, the theory of nonlinear vibrations of systems with many degrees of freedom and, probably, for laser chemistry.

Unfortunately, no experiments have been carried out in which intramolecular nonequilibrium (nonstochasticity) would be reliably assertained under strong MP excitation of molecules. (In any case this refers to experiments with IR pulses with $\tau_{p}=10^{-6}-10^{-7} \mathrm{~s}$ ). Moreover, 
according to direct diagnostic experiments, the stochastization limit for such molecules as $\mathrm{SF}_{6}$ and $\mathrm{CF}_{3} \mathrm{I}$ is very low. The value of $E_{\text {stoch }}$ for the $\mathrm{SF}_{6}$ molecule, for example, does not exceed the value of zero-point vibration energy $E_{Z}$. At first sight this result may seem to be somewhat unexpected. But, as it has been shown in Ref. 8), it is in the energy range $E>4000 \mathrm{~cm}^{-1}\left(4 v_{3}\right)$ that the mechanism of mixing by chain (see Section 5, Ref. 1, Section 2.3) on account of three- and four-frequency Fermi resonances provides effective interaction of the levels of the $\nu_{3}$ mode under excitation with the levels of other modes of the $\mathrm{SF}_{6}$ molecule. At the same time with $E \simeq 3000 \mathrm{~cm}^{-1}\left(3 \nu_{3}\right)$ such interaction is still small.

All this, of course, does not make impossible non-stochasticity for molecules of other types (more complex for example) and especially during their excitation by ultrashort pulses $\tau_{p}=10^{-10}-10^{-11} \mathrm{~s}$. Really, the stochastization time $\tau_{\text {stoch }}$ depends on many conditions: the structure of molecule, the frequencies of its vibrations, the force of intermode interaction (intermode anharmonicities), the excitation leveland probably lies with the picosecond region. If the excitation rate is made higher than the vibrational energy stochastization rate, one can hope, in principle, for observing "mode-selective" effects in MP excitation and subsequent phototransformations of molecules. This possibility has not been studied experimentally yet but its importance is stressed in a number of papers (see Refs. 9, 10).

As far as realization of "bond-selective" IR photochemistry is concerned, of particular interest are large molecules having remote polyatomic functional groups. It is evident that strong vibrations belonging to different remote functional groups will be found rather loosely to each other. So it is not impossible that in MP excitation of the vibrations of one functional group one can attain essentially nonequilibrium energy distribution inside a large molecule during a short time interval and hence a nonstatistical way of photochemical processes.

In connection with this possibility in Ref. 10 an experiment is proposed to observe the nonstatistical behavior of a polyatomic molecule (Figure 6.5) in MP excitation with different functional groups at its ends. Let one functional group $\left(\mathrm{CF}_{3}\right.$, for example) be excited by a powerful $\mathrm{CO}_{2}$ laser pulse. Since this group is not connected directly with the motion of the $R$ radical at the opposite end of the molecular chain, the vibrational perturbation will reach this end in a 


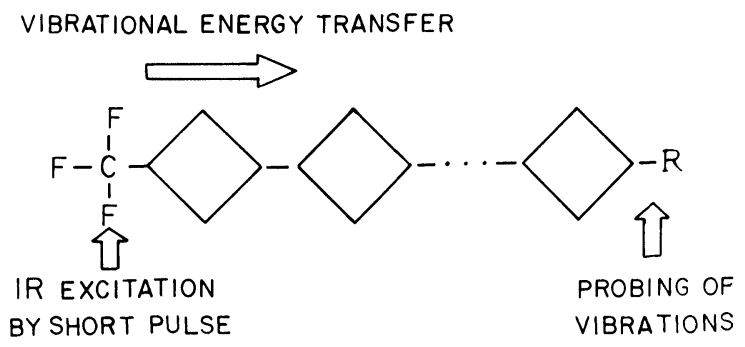

FIGURE 6.5 On the observation of non-equilibrium intramolecular distribution of vibrational energy.

characteristic time $\tau \simeq n / 2 \pi c X$, where $n$ is the number of bonds in the chain which is proportional to the number of atoms, $X$ is the characteristic anharmonicity constant responsible for the interaction of vibrations or the anharmonic coupling of adjacent bonds in the chain. If a molecule is excited by a powerful IR radiation pulse with its duration $\tau_{p}=10^{-10} \mathrm{~s}$, with $X \simeq 1 \mathrm{~cm}^{-1}$ and $n=10$ the results may materially differ from the predictions of the statistical model. Such a difference can be recorded by observing the anti-Stokes RS or IR fluorescence for the functional group at the opposite end of the molecule.

\subsubsection{IR absorption spectrum of a highly excited polyatomic molecules}

The first direct experimental data on the existence of vibrational quasicontinuum for polyatomic molecules as well as on the transition spectrum in it was obtained in experiments ${ }^{11}$ on MP dissociation of molecules in a two-frequency IR field. Comparatively a weak IR field with its $\Omega_{1}$ frequency resonantly excited the molecules to the quasicontinuum, and the second rather intense IR field with its $\Omega_{2}$ frequency detuned from the resonance frequency of unexcited molecules performed MP excitation in the quasicontinuum up to dissociation. The separation of excitation functions between pulses of two different frequencies enables independent research of the molecular transition spectrum in the quasicontinuum. Such a research can be carried out by measuring different physical values, such as absorbed energy, dissociation yield, electronic luminescence intensity. Appropriate experiments were performed with $\mathrm{SF}_{6},{ }^{11-13} \mathrm{SiF}_{4},{ }^{12} \mathrm{OsO}_{4},{ }^{14} \mathrm{CF}_{3} \mathrm{I}^{15}$ The experiments on two-frequency dissociation of the $\mathrm{SF}_{6}$ molecule 
performed in ${ }^{11}$ show that the IR excitation of $\mathrm{SF}_{6}$ gives rise to a quasicontinuous transition spectrum. Quite a similar result was obtained in Refs. 21 and 26 of Chapter 5 from measuring the MP absorption in a two-frequency field. In measuring the absorbed energy in a two-frequency field one should measure first the average energy absorbed separately from the pulses of resonant $\left(\bar{\varepsilon}_{1}\right)$ and nonresonant $\left(\varepsilon_{2}\right)$ steps and then during their joint action $\bar{\varepsilon}_{1+2}$. The value of $\bar{\varepsilon}_{q c}$ determined from the relation

$$
\bar{\varepsilon}_{q c}=\bar{\varepsilon}_{1+2}-\left(\bar{\varepsilon}_{1}+\bar{\varepsilon}_{2}\right)
$$

is the average energy of molecule in the quasicontinuum.

Figure 6.6 shows the frequency dependences of $\bar{\varepsilon}, \bar{\varepsilon}_{1+2}$ and $\bar{\varepsilon}_{q c}$ for the $\mathrm{SF}_{6}$ molecule with the $\Omega_{2}$ frequency shifted to the short-wave side about the $\nu_{3}$ mode band. It may be seen that the value of interest $\bar{\varepsilon}_{q c}$ lies in a wide spectral range that considerably exceeds the band width of the $\nu_{3}$ mode. However, the transition cross-section in the quasicontinuum at the shortwave side is comparatively small $\sigma_{q c} \simeq$ $1-2 \cdot 10^{-21} \mathrm{~cm}^{2}$. It must be noted that the appearance of nonresonant

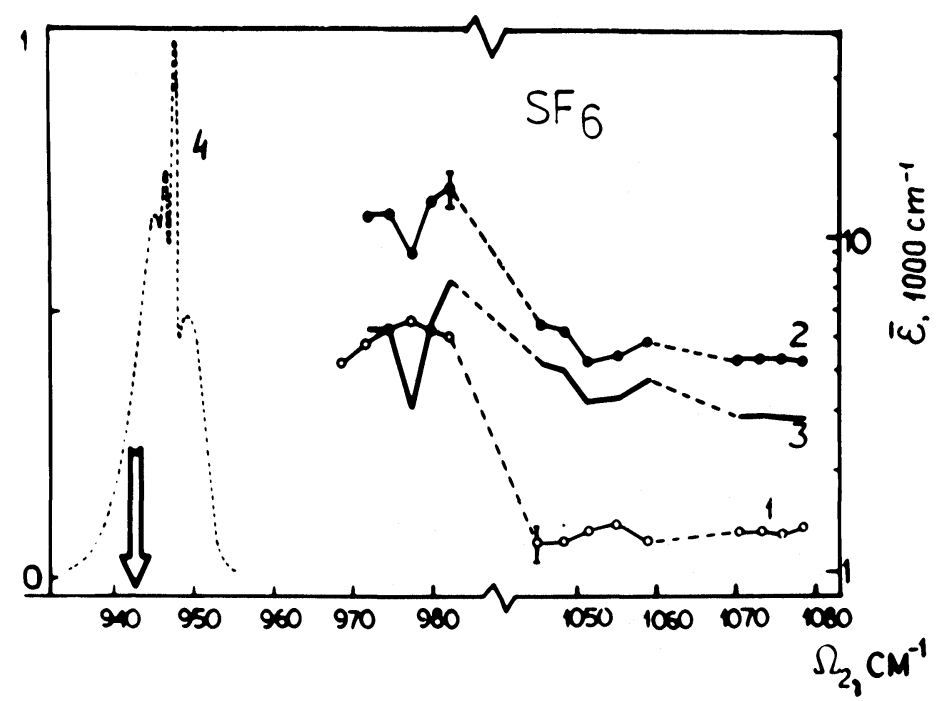

FIGURE 6.6 Dependence of the average number of absorbed quanta in $\mathrm{SF}_{6}$ on nonresonant laser radiation frequency. (1) $\bar{n}_{2} ;$ (2) $\bar{n}_{1+2} ;$; (3) $\bar{n}_{q c}$; (4) linear absorption spectrum of $\mathrm{SF}_{6} ; p_{\mathrm{SF}_{6}}=0.1$ Torr, $\Phi_{1}=0.14 \mathrm{~J} / \mathrm{cm}^{2}, \Phi_{2}=4 \mathrm{~J} / \mathrm{cm}^{2}$. The vertical arrow denotes the resonant radiation frequency $\left(\Omega_{1}=942.38 \mathrm{~cm}^{-1}\right)$. (From Section 5, Ref. 21 ). 
absorption over a wide spectral range during MP excitation of molecules to the quasicontinuum can be also observed in the linearabsorption spectra of MP excited molecules (in spectra of double IR-IR resonance). But because of a small value of $\sigma_{q c}$ the nonresonant absorption from the short-wave side manifests itself in the linear spectra of MP excited molecules very slightly. From the long-wave side the absorption manifests itself more clearly (see Section 6.1.4).

Because of vibration anharmonicity one should expect that the spectrum of the transitions between highly excited levels will be long-wave displaced. For many molecules this manifests itself in IR absorption and IR fluorescence spectra, in Raman spectra (see Chapter 5) and, besides, in frequency dependences of absorbed energy and dissociation yield. Much more clearly the "red" shift manifests itself in two-frequency excitation of molecules. For such small molecules as $\mathrm{SF}_{6}, \mathrm{Oso}_{4}, \mathrm{SiF}_{4}$, for example, this shift may be as large as $50-70 \mathrm{~cm}^{-1}$ with relation to the maxima of the linear absorption spectra (see Figure 6.4). The dependence of the electron luminescence intensity of $\mathrm{OsO}_{4}$ on the second field frequency $\Omega_{2}$ is shown in Figure 6.7. It clearly illustrates that the transition cross-section increases as the frequency is shifted to the long-wave side.

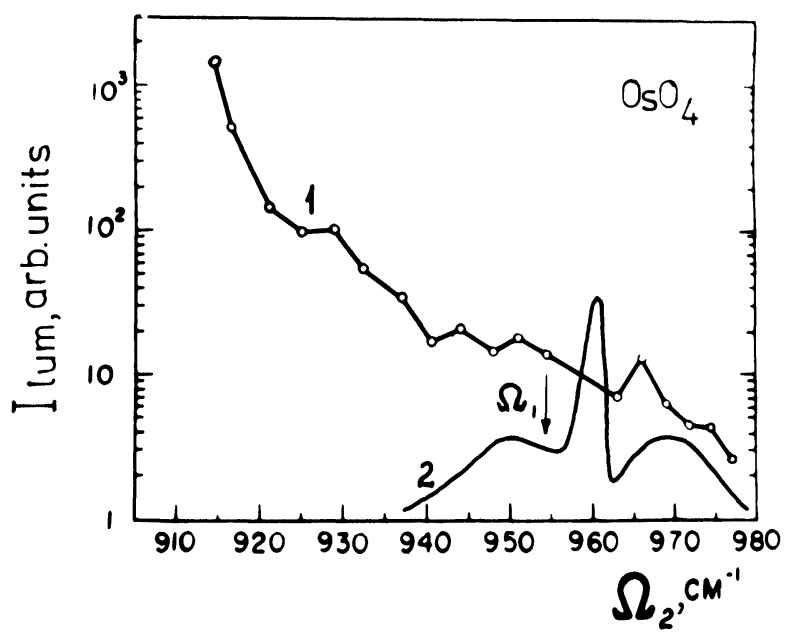

FIGURE 6.7 Dependence of the electronic luminescence intensity of $\mathrm{OsO}_{4}$ on the second field frequency $\Omega_{2}$ (curve 1). $\Phi_{1}=0.24 \mathrm{~J} / \mathrm{cm}^{2} ; \Phi_{2}=0.22 \mathrm{~J} / \mathrm{cm}^{2} ; \Omega_{1}=$ $954.5 \mathrm{~cm}^{-1}$, curve 2-linear absorption spectrum of $\mathrm{OsO}_{4}$. (From Ref. 14). 
Figure 6.8. shows the absorption cross-section in the quasicontinuum for the $\mathrm{SF}_{6}$ molecule as a function of laser energy fluence of resonant field $\Phi_{2}$ at variable values of $\Phi_{1}$ of resonant field. ${ }^{16}$ If the value of $\Phi_{2}$ is fixed and 3-5 photons per molecule are absorbed from the resonant field $\left(\Phi_{1}=0.3 \mathrm{~J} / \mathrm{cm}^{2}\right)$, the value of $\sigma_{q c}$ increases by $20-40$ times. With $\phi_{1}>0.3 \mathrm{~J} / \mathrm{cm}^{2}$, however, there is no increase in $\sigma_{q c}$ any longer since the number of molecules being in resonance with $\Omega_{2}$ field after their interaction with the $\Omega_{1}$ field stops growing. Such behavior of $\sigma_{q c}$ according to Ref. 16 is typical for all the $\Omega_{2}$ frequencies in the range from $944-925 \mathrm{~cm}^{-1}$. The maximum value of MP absorption cross-section in the quasicontinuum observed from the long-wave side of the spectrum can reach for $\mathrm{SF}_{6} \sigma_{q c} \simeq 10^{-18} \mathrm{~cm}^{2}$.

Thus, the observed "red" shifts in the spectra of highly excited molecules as well as in the frequency dependences of absorbed energy and dissociation yield in one- and two-frequency fields are physically clear enough: they directly follow from vibration anharmonicity.

Here it should be noted however that the largest "red" shift can be observed in case of molecules with comparatively a small number

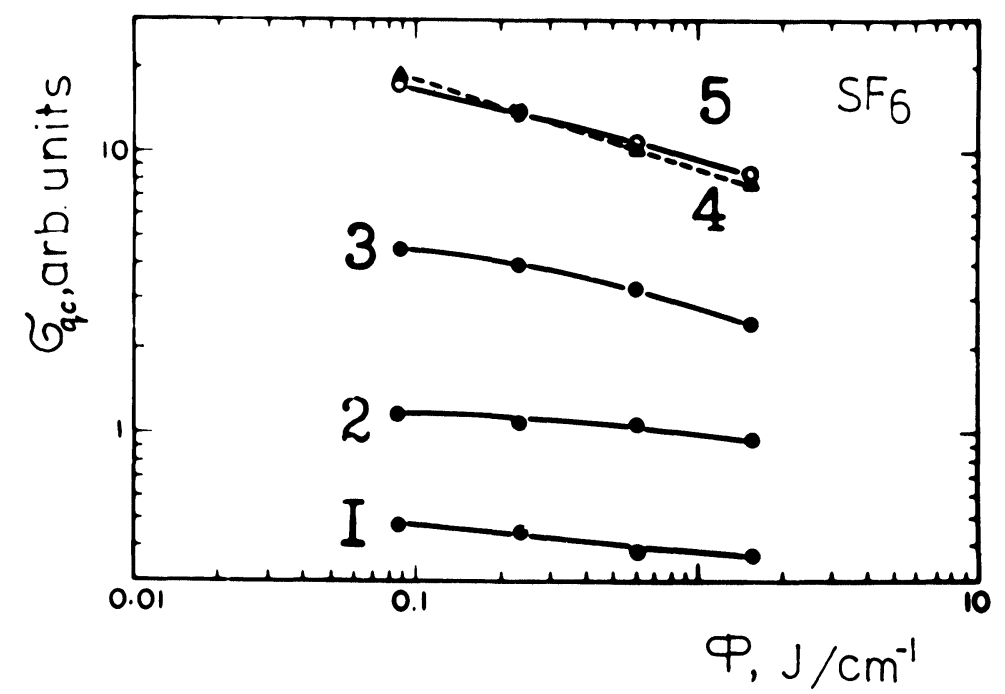

FIGURE 6.8 Dependence of the absorption cross-section of $\mathrm{SF}_{6}$ in the quasicontinuum $\delta_{q c}$ on the energy fluence of nonresonant field $\Phi_{2}$. (1) $\Phi_{1}=0$; (2) $\Phi_{1}=$ $0.016 \mathrm{~J} / \mathrm{cm}^{2}$; (3) $\Phi_{1}=0.073 \mathrm{~J} / \mathrm{cm}^{2}$; (4) $\Phi_{1}=0.34 \mathrm{~J} / \mathrm{cm}^{2}$; (5) $\Phi_{1}=1.72 \mathrm{~J} / \mathrm{cm}^{2} . \Omega_{1}=$ $947.74 \mathrm{~cm}^{-1} ; \Omega_{2}=920.83 \mathrm{~cm}^{-1} ; p=0.2$ Torr, $\tau_{p}=180 \mathrm{~ns}$ (From Ref. 16). 
of atoms, such as $\mathrm{SF}_{6}, \mathrm{OsO}_{4}, \mathrm{SiF}_{4}$, etc. At the same time, for "large" polyatomic molecules the "red" shift may be rather slight or even unobservable. ${ }^{7,17}$ Figure 6.10 shows the spectrum of linear IR absorption and the frequency dependence of the dissociation yield $\beta$ at room temperature for a large 44-atom molecule as $\mathrm{UO}_{2}$ (hfacac) ${ }_{2} \mathrm{THF}^{7}$ These dependences prove to be fully identical. The authors ${ }^{7}$ explain the absence of a shift in the frequency dependence of $\beta$ in this way. The molecule $\mathrm{UO}_{2}$ (hfacac) ${ }_{2} \mathrm{THF}$ at room temperature has an average

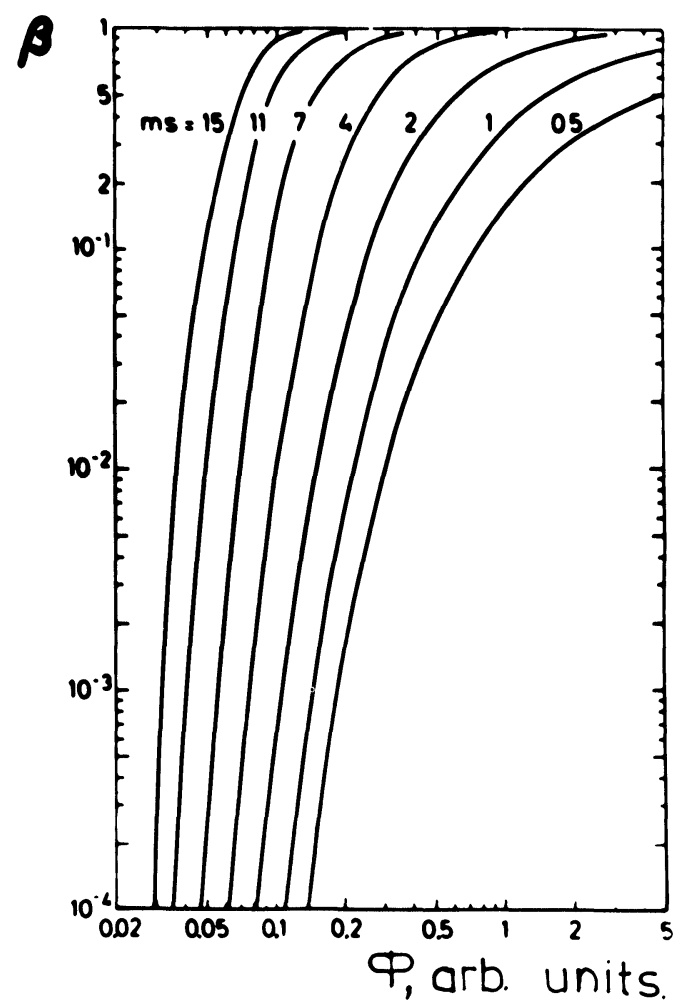

FIGURE 6.9 Theoretical dependences of the dissociation yield $\beta$ of $\mathrm{SF}_{6}$ molecules on laser energy fluence $\phi$. The parameter $m=\frac{1}{2}$ is found from comparing the theoretical and experimental dependences $\bar{\varepsilon}(\Phi)$. The best agreement between the experimental dependence $\beta(\Phi)$ and theory takes place with $m S=1$, where $S$ is the number of vibrational degrees of freedom participating in the process of excitation, hence $S \simeq 2$ (From Ref. 25). 
energy $\tilde{E} \simeq 10000 \mathrm{~cm}^{-1}$ and is already in the region of quasicontinuum with a very high density of vibrational levels. MP absorption comes about due to excitation of the vibrational transition $0 \rightarrow 1$ of the asymmetrical vibration of the group $\mathrm{O}-\mathrm{U}-\mathrm{O}$. Because of fast intramolecular energy transfer the energy of each absorbed IR quantum is distributed over a large number of vibrational degrees of freedom. The asymmetrical mode comes back to the ground state, and the absorbed energy accumulated mainly in a great number of low-frequency modes does not affect materially the spectrum of the transition under excitation. This is also confirmed by the observed linear dependence of absorbed energy on laser energy fluence typical of rather large molecules.

\subsection{Comparison of theory of molecular excitation in the quasicontinuum with experiment}

In Chapter 5, Ref. 2, Section 3.5 some kinetic equations are formulated (3.129) which describe the dynamics of excitation of molecules to vibrational quasicontinuum levels with their unimolecular dissociation taken into account. The solution of the kinetic equations allows finding the average number of absorbed quanta

$$
\bar{n}=\sum_{n} n\left[Z_{n}\left(\tau_{p}\right)-Z_{n}(0)\right]
$$

(see the designations in section 3.5). Further, it enables the dissociation yield during a laser pulse to be determined

$$
\beta_{1}=\sum_{n} \int_{0}^{\tau_{p}} K_{n} Z_{n}(t) d t
$$

The molecules which can be found on the levels above the dissociation limit after the pulse action is over can dissociate after the laser pulse, too, making the following contribution to the dissociation yield

$$
\beta_{2}=\sum_{n>D / \hbar \Omega} Z_{n}\left(\tau_{p}\right)
$$

The total dissociation yield will be $\beta=\beta_{1}+\beta_{2}$. The dissociation rate $K_{n}$ can be calculated with the statistical theory of unimolecular dissociation, RRKM theory, (see Chapter 5, Ref. 1, Section 2.4). The values 
of $\bar{\varepsilon}$ and $\beta$ can be measured experimentally, and so it is convenient to use them to check up theoretical models. However to make the description of the behavior of a molecule in a strong IR field complete, the kinetic equations must be complemented with equations which describe the process of a molecule passing through several lower transitions where the level density is not enough to make cascade resonances possible. Some difficulties the theory encounters in this field, which can be explained by a shortage of spectroscopic information and a necessity of taking into account a lot of multistep and multiphoton transitions, cause the correct selfmatching solution of the whole problem to have not been found at present. Nevertheless one may to describe the experiments on the dynamics of MP excitation at rather strong molecular excitation within the limits of kinetic equations.

Really, direct measurements (see Section 5.3) show that at laser energy fluences when the process of dissociation of molecules becomes appreciable their essential fraction $q$ is excited to the region of quasicontinuum. So one can hope that such characteristics of the process as the average number of absorbed quanta $\bar{n}$, the dissociation yield $\beta$ and the relation between $\bar{n}$ and $\beta$ can be described rather correctly with kinetic equations. For correct correlation of the kinetic model with the experimental results it is necessary in this case to know the cross-sections of successive transitions in the quasicontinuum.

\subsubsection{Transition cross-sections in the quasicontinuum}

There are three different approaches to determining the cross-sections of successive transitions at the present time. The first of them does not make use of any spectroscopic parameters of molecules and is completely based on fitting the results of computation to the results of measurement by varying the unknown parameters entering into the successive transition cross-sections in the kinetic equations. Several different versions of such a simplified empirical approach will be considered below.

The second approach that can be called semiempirical makes use of, first, a more realistic picture of the process of MP excitation, the existence of two molecular ensembles in particular, and, secondly, certain spectroscopic molecular parameters. The model based on such an approach will be considered in section 6.2.2. 
And, finally, the third approach that can be called spectroscopic does not make use of the results of experimental studies into MP processes and is based on considering the intermode resonances of a molecule with involving the anharmonic interaction constants from known spectral data. The first results in this direction are obtained in Ref. 18.

Let us consider now several points of the empirical approach to determining transition cross-sections.

In Refs. 19 and 20 the dynamics of excitation of the $\mathrm{SF}_{6}$ molecule to the quasicontinuum was described by using the following phenomenological dependence of the cross-section on the transition number

$$
\sigma_{n-1, n}=\exp \left(a_{1} n+a_{2}\right)
$$

The comparison was carried out with the dissociation yield measured experimentally during excitation of $\mathrm{SF}_{6}$ in a molecular beam at the $944.2 \mathrm{~cm}^{-1}$ frequency. Good agreement with the dependence observed experimentally can be attained with the following values of the parameters in (6.11): $a_{1}=-42.93 ; a_{2}=0.02936$ (the dimension of $\sigma$ is in $\mathrm{cm}^{2}$ ).

In the ensuing work ${ }^{21}$ however, it was concluded that phenomenological dependence (6.8) could not ensure an agreement between the results of calculation and measurement. In this work the dissociation yield was also measured at the $944.2 \mathrm{~cm}^{-1}$ frequency (at a pressure of 0.2 Torr) with addition of a scavenger of the $\mathrm{H}_{2}$ radical (at a pressure of 1.8 Torr). The comparison with the calculation shows that good agreement takes place with the cross-sections chosen phenomenologically with the use of the formula

$$
\sigma_{n-1, n}=\sigma_{0} n^{-1} \quad\left(\sigma_{0}=5 \cdot 10^{-18} \mathrm{~cm}^{2}\right)
$$

proposed in Refs. 22 and 23. The assumption on the cross-section being independent of the transition number $\left(\sigma_{n-1, n}=\right.$ const $)$ adopted in Ref. 24 turns out not to be able to ensure agreement between theory and experiment.

Some attempts were made in Ref. 25 to compare more completely the results of calculation and measurement with $\mathrm{SF}_{6}$ excited at $944.2 \mathrm{~cm}^{-1}$. Both the dissociation yield and absorbed energy were compared. The results of calculation were compared with the results 
of three experiments. ${ }^{26-28}$ In Ref. 25 the spectrum of molecular transition from an excited state was in the form of Lorentzian contour. For the contour half-width $\sigma(E)$ and for its integral intensity $\left\langle\mu^{2}(E)\right\rangle$ the following phenomenological dependences on molecular vibrational energy were arbitrarily taken

$$
\begin{aligned}
\delta(E) & =B[(E / \Omega)+1]^{c} \\
\left\langle\mu^{2}(E)\right\rangle & =(E / \Omega) \mu_{3}^{2}
\end{aligned}
$$

where $b$ and $c$ are the model parameters, and $\mu_{3}$ is the vibrational dipole moment of the $\nu_{3}$ mode for the $\mathrm{SF}_{6}$ molecule. From comparing the results of calculation and experimental measurement of absorbed energy it was concluded that in case of $\mathrm{SF}_{6}$ the cross-sections of successive transitions were constant.

Thus, the conclusions in all three works on the law of transition cross-section variation differ materially. From this fact it follows that it is not probably this law that can give a key to adequate comparison of theory with experiment. Indeed, in Ref. 76 of Chapter 5, it was assumed that it was necessary to take into account the fact that the fraction of molecules excited to the quasicontinuum differed from unity although it was essential. In Ref. 76 of Chapter 5 the crosssections of successive transitions were calculated from the formulas given in Chapter 5, Ref. 1 (Section 2.3.4), where a version was used based on superposition of three Lorentzial contours corresponding to the $P, Q$ and $R$-branches. Such a choice of cross-sections proves to be quite sufficient for obtaining good agreement between theory and experiment. The principles of comparison between the results of calculating the excitation dynamics and the experimentally measured values had to be revised in this case as compared to Refs. 18-22.

Before considering the approach developed in Ref. 34 of Chapter 5 we must note one important factor. The use of phenomenological dependences like (6.8) and (6.9) for the cross-sections of successive transitions in the quasicontinuum hardly has any sense in comparison between theory and experiment for different frequencies of exciting radiation. Even if it is possible to choose its dependence for any frequency, it will be rather difficult to obtain illustrative physical information. At the same time it is quite clear that the transition cross-sections at different frequencies must be coupled physically with one another. The formulas obtained in Chapter 5, Ref. 1 (Section 
2.3) provide such a coupling. In the simplest case the cross-sections at all frequencies and all transitions in the quasicontinuum can be expressed only through two parameters-the average anharmonicity constant and average Fermi-interaction constant-having clear physical sense. It is apparently of independent interest to determine these parameters from comparing the results of calculation of the molecular excitation dynamics with the results of experimental measurement of absorbed energy and dissociation yield.

\subsubsection{Semiempirical model of IR excitation and dissociation}

In Ref. 25 referred to in Section 6.2.1 it was impossible to get agreement between theory and experiment for the $\mathrm{SF}_{6}$ molecule simultaneously for the both dependences of the dissociation yield $\beta$ and adsorbed energy $\bar{\varepsilon}$ on laser pulse energy fluence $\Phi$. It turns out that, if by choosing proper parameters one can get agreement between the theoretical and experimental dependences on absorbed energy, the theoretical dependence of the dissociation yield on $\Phi$ comes to saturation much faster than the experimental one (see Figure 6.10). For a potential cause in Ref. 25 it had to be assumed that not all the vibrational degrees of freedom of the $\mathrm{SF}_{6}$ molecule but only two of them took part in the process of its excitation.

This assumption is synthetic since it does not agree with the experimental results. The authors of Ref. 76 in Chapter 5 stressed on the necessity of taking into account the division of molecules into two ensembles. The lower "cold" ensemble consists of molecules the excitation of which can be neglected. The upper "hot" ensemble the relative population of which is the $q$ factor (see Section 5.3) is described by the distribution that qualitatively corresponds to the distribution resulting from the solution of kinetic equations. As the laser pulse energy fluence increases, the ratio between the total populations of the lower and upper ensembles changes, the upper ensemble population therewith increasing, which must be described by a monotonous increase of the $q(\Phi)$ dependence. The inclusion of the division of molecules into two ensembles has allowed the results of theory and experiment to be in satisfactory agreement.

According to Section 5 (Ref. 76) the dependences $\beta_{\text {theor }}(\Phi)$ and $\bar{\varepsilon}_{\text {theor }}(\Phi)$ calculated with the use of kinetic equations are evidently related to their associated experimental dependences in the following 


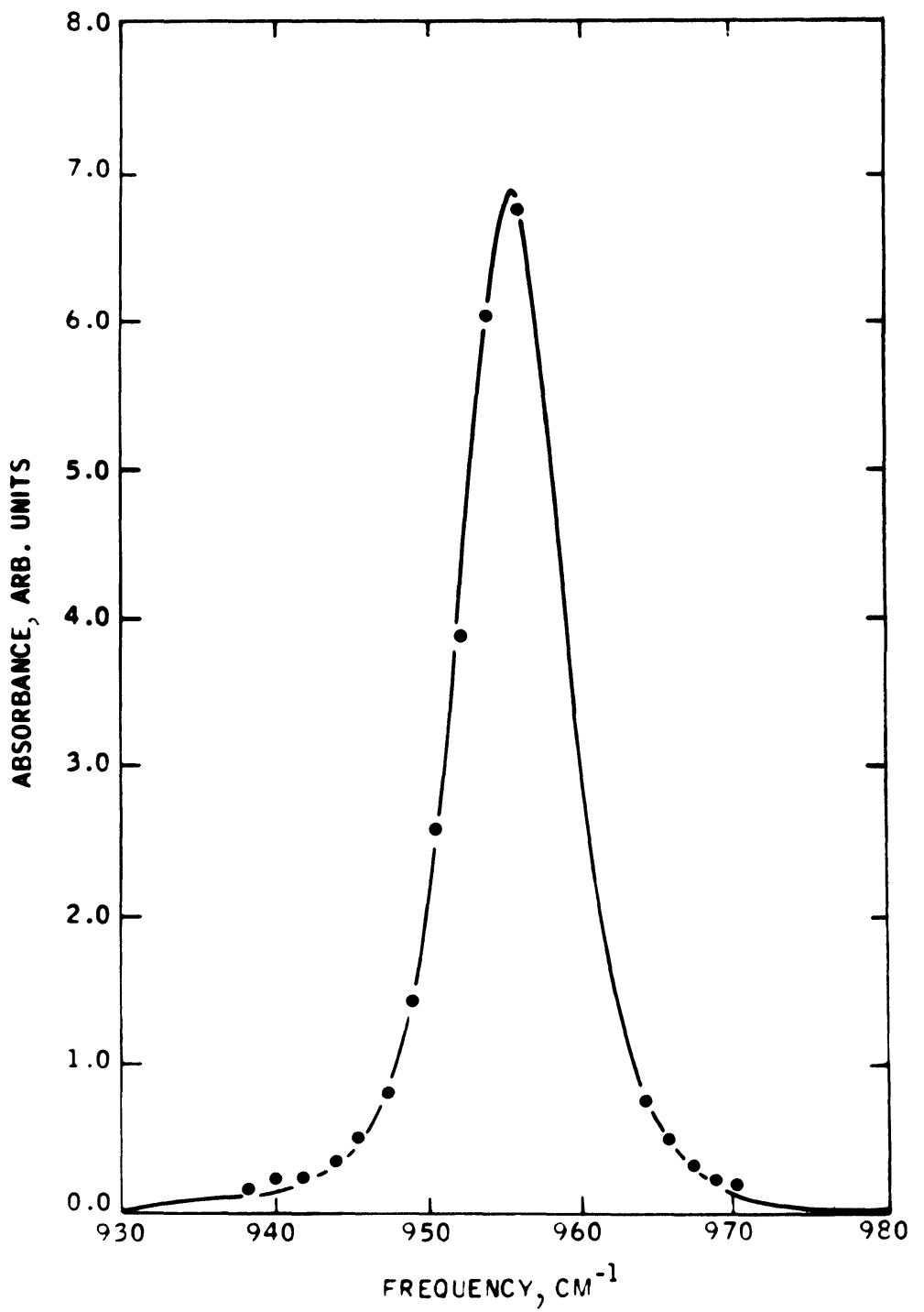

FIGURE 6.10 Frequency dependence of the dissociation yield (points) and the spectrum of linear absorption of the $\mathrm{UO}_{2}(\mathrm{hfacac})_{2} \mathrm{THF}$ molecule at room temperature (From Ref. 7). 
way

$$
\begin{aligned}
\beta_{\text {theor }}(\Phi) & =\beta_{\text {experim }}(\Phi) / q(\Phi) \\
\bar{\varepsilon}_{\text {theor }}(\Phi) & =\bar{\varepsilon}_{\text {experim }}(\Phi) / q(\Phi)
\end{aligned}
$$

As it may be seen from (6.12), the ratio of dissociation yield to absorbed energy $\beta / \bar{\varepsilon}$ must not depend on the $q$ factor. It is this ratio that can be used in comparing calculation and experiment over a wide range of both energy fluence $\Phi$ and laser radiation frequency $\Omega$. It should be noted that such comparison does not call for the knowledge of the value of $q$. Only the experimentally proved fact of existence of two molecular ensembles is applied here. It was for this reason that the theoretical model pretending to agree with experiment by the value of the $\beta / \bar{\varepsilon}$ ratio was called semiempirical in Ref. 76 of Chapter 5 . It is evident that, when the semi-empirical model provides good agreement with experiment, the dependences of the $q$ factor on both laser pulse frequency and energy fluence can be found. Thus, the semi-empirical model can be considered as one more method, in addition to those discussed in Section 5.3, to determine the $q$ factor.

Now, in accordance with Section 5, Ref. 76, we are going to consider the comparison of the semi-emprical model with experiment for the $\mathrm{CF}_{3} \mathrm{I}$ molecule. The comparison was performed with the dimensionless value of quantum dissociation efficiency $\varphi=D / \beta / \bar{\varepsilon}$, where $D$ is the dissociation limit of the $\mathrm{CF}_{3} \mathrm{I}$ molecule. As it follows from the results of Section 2.3.4 in Chapter 5, Ref. 1 the average anharmonicity constant $\langle X\rangle$ and the average constant of three-frequency Fermi interaction $V$ were considered as variable parameters of the model which determined the cross-sections of successive transitions.

A rigorous approach to determining the cross-sections of transitions must undoubtedly be based on taking into account rotations. Since for the excited $\nu_{1}$ band of the $\mathrm{CF}_{3} \mathrm{I}$ molecule the selection rule $\Delta K=0$ is fulfilled with respect to the projection of momentum onto the molecular axis, the contour of molecular absorption from an excited vibrational-rotational state averaged by $K$ is formed with the superposition of three Lorentzian contours corresponding to the transitions in the $P$-, $Q$ - and $R$-branches. A rigorous allowance for rotations causes the equations to grow considerably. But, as shown in Section 5 , Ref. 76 , the rotations can be allowed for in an averaged way by solving the kinetic equations for rotational states $J_{0}$ with a maximum initial population (for $\mathrm{CF}_{3} \mathrm{I}$ with $T=293 \mathrm{~K} J_{0}=50$ ) and taking into 
account all the three branches. The same averaging can be also performed relative to "hot" branches assuming that the molecules starts not from the ground state but from a level being equal to the initial average vibrational energy (for $\mathrm{CF}_{3} \mathrm{I}$ with $T=293 \mathrm{~K} E_{0} \simeq$ $400 \mathrm{~cm}^{-1}$ ). The tests carried out in Chapter 5, Ref. 76 for integrating the full system of kinetic equations that allows for both the distribution over rotational levels and the initial distribution over vibrational levels yielded calculated values of $\bar{\varepsilon}$ and $\beta$ which differed a little (3\%) from the results of averaged calculations. The calculations performed in Section 5, Ref. 76 for the $\mathrm{CF}_{3} \mathrm{I}$ molecule show that the theoretical and experimental dependences of the quantum dissociation efficiency on radiation frequency with fixed energy fluence coincide within the limits of experimental errors with the following parameter values: $\langle X\rangle=3.8 \pm 0.2 \mathrm{~cm}^{-1}, V=0.73 \pm 0.03 \mathrm{~cm}^{-1}$. Quite a high accuracy of determination of two parameters is indicative in favor of two factors. First, it has been found that the results of model calculation, if they are considered in total over the entire frequency range, are rather sensitive to the choice of cross-sections. Second, the fact that the cross-sections at all frequencies can be described with the use of only two variable parameters shows that the representations on the transition spectrum in the quasicontinuum developed in Chapter 5, Ref. 1 (Section 2.3) are correct as a whole. The experimental and theoretical frequency dependences of the quantum dissociation efficiency $\varphi$ are given in Figure 6.11.

It stands to reason that the calculated values of dissociation yield $\beta_{\text {theor }}$ and absorbed energy $\bar{\varepsilon}_{\text {theor }}$ with the values of $\phi_{\text {theor }}$ and $\phi_{\text {experim }}$ coincident turn out to be higher than their corresponding experimental values $\beta_{\text {experim }}$ and $\varphi_{\text {experim. }}$. From the ratio of any of these pairs it was possible to determine the frequency dependence of the $q$ factor for the $\mathrm{CF}_{3} \mathrm{I}$ molecule (see Figure 5.21).

In Section 5, Ref. 2 (Figure 4.10) are given the theoretical and experimental dependences of the quantum dissociation efficiency of $\mathrm{CF}_{3} \mathrm{I}$ on the laser pulse energy fluence $\phi$ at the $1074.6 \mathrm{~cm}^{-1}$ frequency. These dependences coincide within the limits of measurement error from $\phi \geqslant 0.7 \mathrm{~J} / \mathrm{cm}^{2}$. At lower values of laser pulse energy fluence some discrepancy between the calculated and experimental curves may be caused (according to Chapter 5 , Ref. 76) by the contribution of collisional processes to dissociation not allowed for by the kinetic equations. This possibility is under discussion below in Section 6.3. 


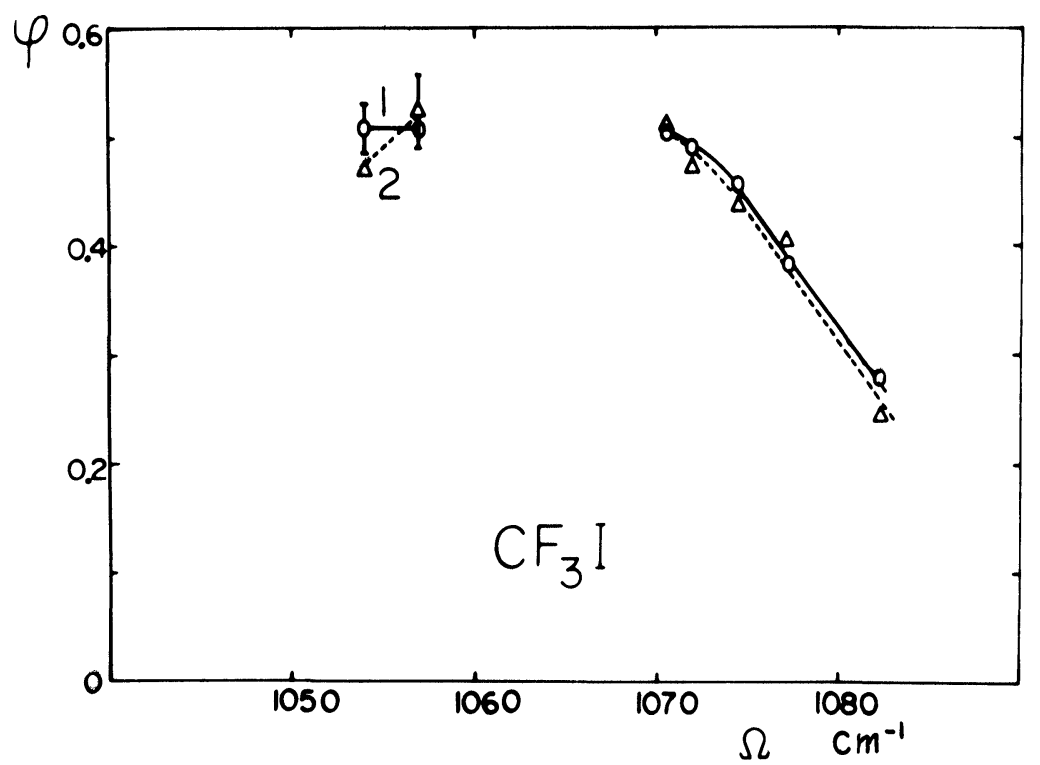

FIGURE 6.11 Frequency dependences of the quantum efficiency of dissociation of the $\mathrm{CF}_{3} \mathrm{I}$ molecule: (1) experiment; (2) semi-empirical model (From Section 5, Ref. 76).

At the same time, the place where the theoretical and experimental curves are well coincident corresponds apparently to the situation when the basic contribution to dissociation is determined by the collisionless process of molecule-radiation interaction. The dependence of the $q$ factor on the laser pulse energy fluence for the $\mathrm{CF}_{3} \mathrm{I}$ molecule calculated from the ratio $\left(\bar{\varepsilon}_{\text {experim }} / \bar{\varepsilon}_{\text {theor }}\right)$ is given in Figure 5.20. It agrees quite well with the values of $q$ obtained by other methods.

Thus, the application of the semi-empirical model in combination with the representations on the transition spectrum in the quasicontinuuum allows a good agreement with experiment. In connection with further potential applications of the semi-empirical model the following notes should be made.

1. Unambiguous interpretation of the absorbed energy values measured in experiments is possible only when the radicals formed by the process of IR photodissociation do not absorb radiation or absorb its negligible portion. This condition, however, is not always 
fulfilled because in some cases the resultant radicals, in their turn, dissociate.

2. The measured values of dissociation yield cannot be always interpreted unambiguously either. A characteristic example of this is the $\mathrm{SF}_{6}$ molecule. It is apparently the process of recombination that is vital to this molecule. ${ }^{29,30}$ It is also of importance to know how to separate the dissociation yield on account of molecule-radiation interaction only from that on account of collisions. As it may be seen from Figure 5.20, the contribution of collisional processes for the $\mathrm{CF}_{3} \mathrm{I}$ molecule at comparatively high values of dissociation yield is obviously not large. But, since small dissociation yields are usually measured, the methods of direct radical diagnostics become of particular importance.

3. The values of average absorbed energy $\bar{\varepsilon}$ and primary dissociation yield $\beta$ in the above case of the $\mathrm{CF}_{3} \mathrm{I}$ molecule were used as experimentally measured parameters to construct a semi-empircal model of MP excitation. For this purpose, however, other combinations of experimentally measured parameters can be used as well. For example, when an excited electronic state of the molecule lies below the dissociation limit of the ground electron state, vibrational MP excitation can bring about electronic excitation of the molecule. In this case visible or UV luminescence arises, and its value, like the $\beta$ value, can be used as a parameter of semi-empirical model. The model thus constructed for the $\mathrm{OsO}_{4}$ molecule, for example, provides quite satisfactory agreement with experiment both at one- and two-frequency excitation. ${ }^{31}$

4. In every case it is necessary to consider specifically the Fermi resonances of the given molecule since the law of spectral width variation with molecular vibrational energy must be governed by the multiplicity of the Fermi resonances responsible for the formation of the transition quasi-continuum (see Section 5, Ref. 1, Section 2.3).

5 . When the semiempirical model gives good agreement with experiment, it is possible to find the dependence of the $q$-factor both on the laser pulse frequency and energy fluence. Thus, the semiempirical model can be considered as another method of determination of the $q$-factor, in addition to the methods concerned in Section 5.3.

6. In calculating the dynamics of excitation and dissociation with the use of kinetic equations it is quite reasonable to use the version when the distribution over rotational levels and thermal distribution 
over lower vibrational levels are considered in an averaged manner. But sometimes control tests are probably required as it is done in Section 5, Ref. 76 where these distributions are allowed for precisely.

\subsection{Vibrational energy distribution at IR-MP excitation of molecules}

The form of vibrational distribution is one of the most important points in the problem of IR-MP excitation of molecules. This is connected with the following. First of all, the form of distribution function contains information on the very process of IR MP excitation. So studies into vibrational distribution can help, first, to specify the excitation mechanisms and, secondly, to determine the parameters of high vibrational molecular states. Besides, the high-energy distribution tail determines the chacteristics of unimolecular phototransformations of the ensemble of MP excited molecules, for example MP dissociation.

In this and foregoing articles (Section 5, Refs. 1 and 2) we have already noted that there are two different points under discussion: absorbed energy distribution inside a molecular ensemble and internal energy distribution of an excited molecule over its vibrational degrees of freedom. In this section consideration will be given only to intermolecular distribution. The point on intramolecular distribution is considered by us quite sufficiently in Chapter 5, Ref. 1 (Section 2.3) and in Section 6.1 of the present paper.

In Section 6.3.1 we are going to discuss the experimental results which reflect the specific features of intermolecular distribution, then (in Section 6.3.2) discuss the factors responsible for the formation of two ensembles during MP excitation and, finally, in 6.3.2 we shall consider the peculiarities of vibrational distribution in the upper molecular ensemble.

\subsubsection{Features of intermolecular distribution nonequilibrium}

The intermolecular vibrational energy distribution formed under IRMP excitation $F\left(E_{\mathrm{vib}}\right)$ is materially nonequilibrium. This conclusion obviously follows from comparing the spectral characteristics of IRMP excited molecules with the characteristics of equilibrium-excited (thermally excited) molecules. Indeed, the spectra of IR absorption, IR fluorescence, spontaneous Raman scattering and CARS produced by different methods of spectroscopic diagnostics essentially differ 
from their associated thermal spectra (see Figure 4.9 in Section 5, Ref. 2, Figure 5.10, 14 and 15). From these spectra (particularly illustrative in Figure 4.9 (Section 5, Ref. 2) and Figure 5.15) it follows that the first most important feature of intermolecular distribution non-equilibrium is the division of molecules into two ensembles: "cold" molecules remaining in the system of lower vibrational levels and "hot" molecules excited to high vibrational states (see expression 4.9 in Section 5, Ref. 2). Quite a number of indirect data on meaurement of absorbed energy $\bar{\varepsilon}$, dissocation yield $\beta$, electronic luminescence intensity and some other characteristics of IR-MP processes is indicative on the division of the molecules into two ensembles. This follows quite clearly from correlating the measured results of IR-MP dissociation yield and absorbed energy. Figure 6.12 illustrates the dependence of the primary dissociation yield of the $\mathrm{CF}_{3} \mathrm{I}$ molecule on absorbed energy $\bar{\varepsilon}$ (curve 1). Curve 3 stands for the results of calculation under the assumption of the Boltzmann distribution function $F\left(E_{\mathrm{vib}}\right)$ (Section 5, Ref. 77). The comparison of curves 1 and 3, for example, shows that for the average vibrational energy of molecules $\bar{E}=$ $7 \cdot 10^{3} \mathrm{~cm}^{-1}$ the calculated dissociation yield $\beta=4 \cdot 10^{-3}$ whereas the measured yield at the same value of absorbed energy $\beta=0.1$. It can be easily seen that at lower values of $\bar{E}$ the discrepancy gets greater. The main cause of such a discrepancy between the experimental and calculated dependences is that no account is taken of the division of molecules into two ensembles. For correct comparison the fraction of molecules $q$ excited to the upper ensemble should be known. In Figure 6.12 curve 3 denotes the dependence of the dissociation yield of the molecules from the upper ensemble $\beta_{q}=\beta / q$ on their average excitation level $\bar{\varepsilon}_{q}=\bar{\varepsilon} / q$ (Section 5, Ref. 77 ). It may be seen that the discrepancy between experiment and calculation is much smaller here. Nevertheless, in the region of small values of $\bar{\varepsilon}_{q}$ the value of $\beta_{q}$ at MP excitation is much smaller than it must follow from the equilibrium Boltzmann distribution. On the contrary, at rather high $\bar{\varepsilon}_{q}$ MP excitation results in a high dissociation yield. Such a comparison is, of course, just qualitative but it allows seeing the difference of the distribution in the upper ensemble from the Boltzmann one that is another feature of nonequilibrium in intermolecular vibrational distribution. The distribution function of "hot" molecules has a steeper high-energy tail and is narrower than the Boltzmann function. Quite a similar result follows from experiments with many other molecules and, besides, 


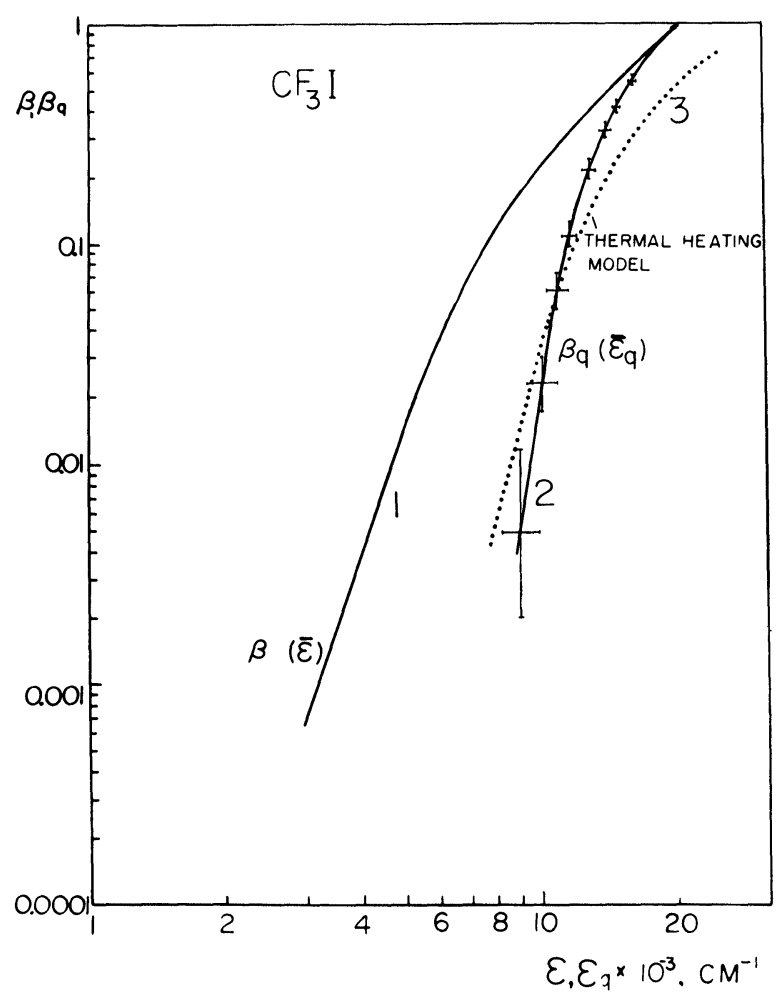

FIGURE 6.12 Dependences of the dissociation yield of $\mathrm{CF}_{3} \mathrm{I}$ on absorbed energy: (1) dependence $\beta(\bar{\varepsilon})$; (2) dependence $\beta_{q}\left(\bar{\varepsilon}_{q}\right)$; (3) calculation with the use of the thermal model (From Section 5, Ref. 77).

corresponds to theoretical representations (see section 6.3.3). It should be noted that comprehensive experimental study of vibrational energy distribution for different molecules as well as with the use of IR pulses of different duration and frequency remains a very urgent task. Great hopes here are set first of all on modern methods of spectroscopic diagnostics, such as IR fluorescence and Raman scattering (see Section 5.2).

\subsubsection{Causes of the formation of two molecular ensembles}

As it has been often noted, some difficulties in calculating the dynamics of excitation of lower transitions connected with a deficit of spectro- 
scopic information and the necessity of taking into account a lot of multistep and multiphoton transitions cause the direct theoretical determination of the $q$ factor which would be adequate to the available experimental results to be hampered greatly. Therefore we shall restrict ourselves just to summing up the available experimental results which somehow clear up the physical cause of the effect concerned. These results were obtained in experiments in which the number of gas-kinetic collisions of molecules changed during a laser pulse. One possibility here is the variation of pressure in the cell either by changing the parent gas pressure (Section 5, Ref. 25) or by diluting the absorbing gas molecules with the buffer gas molecules (Section 5, Ref. 48). Another possibility the variation of laser pulse duration with its energy fixed (Section 5, Ref. 47). In the both cases the maximum value of $p \tau_{p}$ (the product of pressure by pulse duration) must undoubtedly comply with the conditions when during the interaction with the radiation field the number of gas-kinetic collisions is large.

As the results of numerous experimenta show (see Section 5.3, Table 5), under collisional conditions the average number of absorbed quanta (or dissociation yield) can considerably exceed the value measured under collisionless excitation. So we can assume that the efficiency of molecular excitation to the quasi-continuum depends on the initial energy state of this molecule. Some molecular states populated at the temperature of the experiment can be excited to the quasicontinuum rather effectively, other states are excited less effectively. Under collisions the molecules relax to effectively excited levels with the result that, in principle, all the molecules can be excited to the quasicontinuum.

The statement made is trivial and rather common by character. Nevertheless, there is still no theory that would be able to explain the observed values of the $q$ factor at a quantitative level and point to the states which can be effectively excited to the quasicontinuum. It must be noted, however, that the increase of absorption with an increase in the value of $p \tau_{p}$ is usually related to the process of rotational relaxation which is quite natural.

\subsubsection{Vibrational distribution in the upper molecular ensemble}

The distribution in the upper ensemble set up by laser pulses is determined by the solution of kinetic equations Section 5, Ref. 2 
(Section 3.5). We have already considered the basic properties of these solutions. As seen from Figures 3.12 and 3.13, the distribution is belled in shape and concetrated near the average value. The fact that the distribution in the upper ensemble has really such a shape also follows from the spectra of IR absorption, Raman scattering and IR fluorescence of excited molecules (see Figures 5.10, 14 and 15).

When the laser pulse action is over there are two process taking place. First, due to the energy exchange between the upper and lower ensembles they merge together. It is in this way that the time variation of spectra of excited molecules is interpreted. The second relaxation process after the laser pulse action is attaining the Boltzmann distribution in the upper ensemble. As noted in Section 5, Ref. 76 it is this process that can contribute materially to dissociation at relatively low yields.

Really, let us consider the ratio between the distribution calculated with kinetic equations and the Boltzmann distribution at similar values

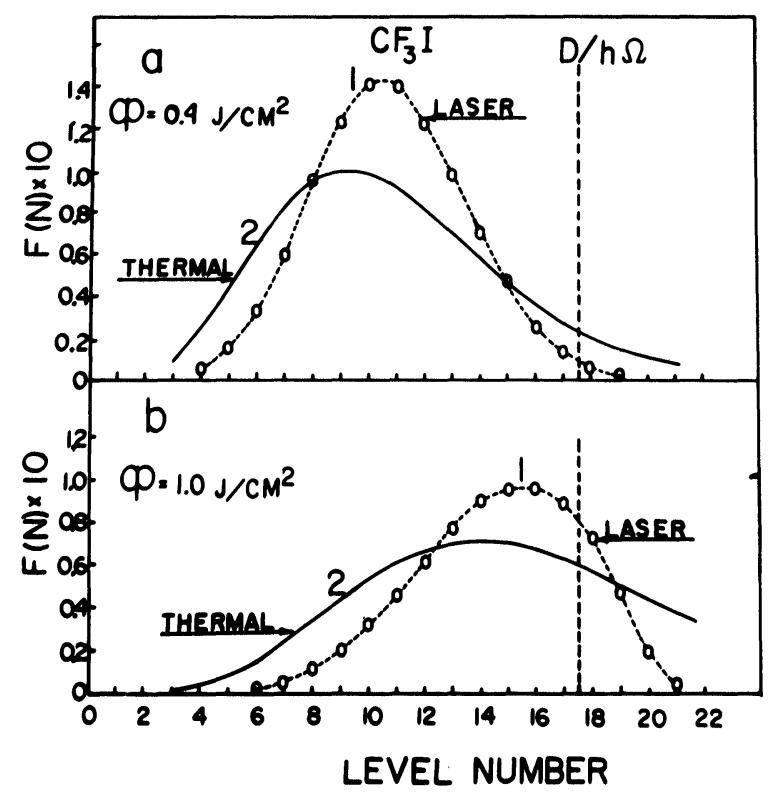

FIGURE 6.13 Comparison of the vibrational distribution in the "hot" ensemble (curves 1) with the thermal one (curves 2) for the $\mathrm{CF}_{3}$ I molecule. (a) $\bar{\varepsilon}_{q}=10500 \mathrm{~cm}^{-1}$; (b) $\bar{\varepsilon}_{q}=16000 \mathrm{~cm}^{-1}$. (From Chapter 5, Ref. 76). 
of absorbed energy for the $\mathrm{CF}_{3} \mathrm{I}$ molecule (see Section 5, Ref. 76). These distributions are presented in Figure 6.12 for two values of laser pulse energy fluence. It may be seen that at comparatively low energy fluences $\phi=0.4 \mathrm{~J} / \mathrm{cm}^{2}$, when the experimentally observed dissociation yield $\beta \simeq 2 \cdot 10^{-2}$, the Boltzmann distribution tail near the dissociation limit is much higher than that of calculated distribution (curves 1). If the laser pulse energy fluence is increased to $\phi=1 \mathrm{~J} / \mathrm{cm}^{2}$, when the experimental dissociation yield $\beta \simeq 0.2$, the calculated distribution tail near the dissociation limit is higher than that of Boltzmann distribution (curves 2). Thus, if we turn our attention again to Figure 4.10 in Chapter 5, Ref. 2, where the calculated and experimental values of quantum dissociation yield $\varphi$ are correlated, we can understand why theory and experiment are well consistent in case of comparatively high values of $\Phi$, and the discrepancy in the region of comparatively low values of $\Phi$ can be qualitatively explained (Section 5, Ref. 76) by the contribution of collisions to dissociation which tend to establish the Boltzmann distribution in the ensemble of highly excited molecules.

The Boltzmann distribution introduced for MP excitation in Ref. 32 is quite good approximation for vibrational energy of the upper ensemble of molecules. The application of this model, for example, to interpret the spectra of IR fluencescence (Section 5, Ref. 66) and UV absorption (Section 5, Ref. 55) corresponding to hot ensembles can produce quite satisfactory results.

\section{References}

1. H. S. Kwok, E. Yablonovitch and N. Bloembergen, Phys. Rev. A23, 3094 (1981).

2. N. Bloembergen and E. Yablonovitch, Phys. Today 31, 23 (1978).

3. J. G. Black, E. Yablonovitch, N. Bloembergen and S. Mukamel, Phys. Rev. Lett. 38, 1131 (1977).

4. P. I. Robinson and K. A. Holbrook, Unimolecular Reactions, (Wiley, London, 1972).

5. R. Hall and A. Kaldor, J. Chem. Phys. 70, 4027 (1979).

6. A. Kaldor, R. B. Hall, D. H. Cox, J. A. Horsley, P. Rabinovitch and G. M. Kramer, J. Am. Chem. Soc. 101, 4465 (1979).

7. D. M. Cox and J. A. Horsley, J. Chem. Phys. 72, 814 (1980).

8. V. N. Bagratashvili, Yu. G. Vainer, V. S. Doljikov, S. F. Koliakov, A. A. Makarov, L. P. Malyavkin, E. A. Ryabov, E. G. Sil'kis and V. D. Titov, Zh. Eksp. Teor. Fiz. (Russian) 80, 1008 (1981; Sov. Phys.-JETP, 53, 512 (1981).

9. A. Kaldor and J. A. Horsley, Proc. 22nd Annual Technical Symposium of the Society of Photooptical Instrumental Engineers, San Diego, California (1978). 
10. V. S. Letokhov, "Laser Spectroscopy IV". Eds H. Walther and K. W. Rothe, Springer Series in Optical Sciences, Vol. 21 (Springer-Verlag, Berlin, Heidelberg, New York, 1979) p. 504.

11. R. V. Ambartzumian, Yu. A. Gorokhov, V. S. Letokhov, G. N. Makarov, A. A. Puretzky and N. P. Furzikov, Pis'ma Zh. Eksp. Teor. Fiz., (Russian) 23, 217 (1976); Sov. Phys. -JETP Lett. 23, 194 (1976).

12. V. M. Akulin, S. S. Alimpiev, N. V. Karlov, A. M. Prokhorov, V. G. Sartakov and E. M. Khokhlov, Pis'ma Zh. Eksp. Fiz. (Russian) 25, 428 (1977).

13. M. C. Gover and T. K. Gustafson, Optics Comm. 23, 69 (1977).

14. R. V. Ambarztumian, V. S. Letokhov, A. A. Makarov and A. A. Puretzky, Optics Comm. 25, 69 (1978).

15. S. S. Alimpiev, N. V. Karlov, A. M. Prokhorov, B. G. Sartakov and E. M. Khokhlov, Kvantovaya Elektr. (Russian) 6, 2597 (1979).

16. G. P. Quigley, Optics Lett. 4, 84 (1979).

17. D. S. King, Dynamics of the Excited States, ed. by K. P. Lawly, (Wiley, London, 1982) p. 14.

18. A. A. Makarov and V. V. Tyakht, Zh. Eksp. Teor. Fiz. (Russian) 83, 502 (1982).

19. E. R. Grant, M. J. Coggiola, Y. T. Lee, P. A. Schultz, Aa. S. Sudbo and Y. R. Shen, Chem. Phys. Lett. 52, 595 (1977).

20. E. R. Grant, P. A. Schulz, Aa. S. Sudbo, M. J. Coggiola, Y. T. Lee and Y. R. Shen, Laser Spectroscopy III, Eds. J. L. Hall and J. L. Carlsten, Springer Series in Optical Sciences, Vol. 7 (Springer-Verlag, Berlin, Heidelberg, New York, (1977) p. 94.

21. A. C. Baldwin, J. R. Barker, D. M. Golden and N. Van den Berg, Chem. Phys. Lett. 62, 179 (1979).

22. M. Quack, J. Chem. Phys. 69, 1282 (1978).

23. M. Quack, J. Chem. Phys. 70, 1069 (1979).

24. J. L. Lyman, J. Chem. Phys. 67, 1868 (1977).

25. W. Fuss, Chem. Phys. 36, 135 (1979).

26. F. Brunner, T. P. Cotter, K. L. Kompa and D. Proch, J. Chem. Phys. 67, 1547 (1977).

27. F. Brunner and D. Proch, J. Chem. Phys. 68, 4936 (1978).

28. W. Fuss and T. P. Cotter, Appl. Phys. 12, 265 (1977).

29. Yu. R. Kolomijsky, A. R. Kukudzhanov and E. A. Ryabov, Kvantovaya Elektr. (Russian) 7, 1499 (1980).

30. D. Tal, U. P. Oppenheim, G. Koren and M. Okon, Chem. Phys. Lett. 48, 67 (1977).

31. A. A. Makarov, G. N. Makarov, A. A. Puretzky and V. V. Tyakht, Appl. Phys. 23, 391 (1980).

32. N. Bloembergen and E. Yablonovitch, "Laser Spectroscopy III". Eds. J. L. Hall and J. L. Carlsten, Springer Series in Optical Sciences, Vol. 7 (Springer-Verlag, Berlin, Heidelberg, New York, 1978) p. 362. 